Portland State University

PDXScholar

1989

\title{
An analysis of the inhibitory effects of linolenic acid upon photosystem II of higher plants
}

Mark Edward Iven

Portland State University

Follow this and additional works at: https://pdxscholar.library.pdx.edu/open_access_etds

Part of the Environmental Chemistry Commons

Let us know how access to this document benefits you.

\section{Recommended Citation}

Iven, Mark Edward, "An analysis of the inhibitory effects of linolenic acid upon photosystem II of higher plants" (1989). Dissertations and Theses. Paper 3893.

https://doi.org/10.15760/etd. 5763

This Thesis is brought to you for free and open access. It has been accepted for inclusion in Dissertations and Theses by an authorized administrator of PDXScholar. Please contact us if we can make this document more accessible: pdxscholar@pdx.edu. 
AN ABSTRACT OF THE THESIS OF Mark Edward Iven for the Master of Science in Chemistry presented February 2, 1989.

Title: An Analysis of the Inhibitory Effects of Linolenic Acid Upon Photosystem II of Higher Plants.

APPROVED BY THE MEMBERS OF THE THESIS COMMITTEE:

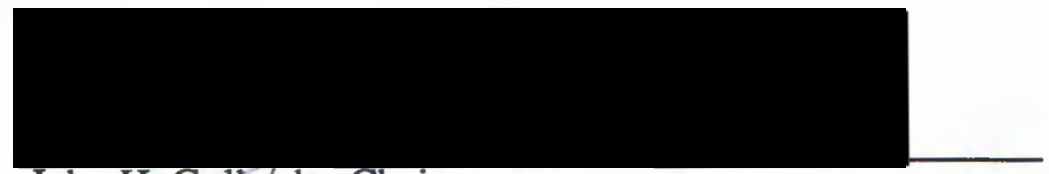

John H. Gotbeck, Chair
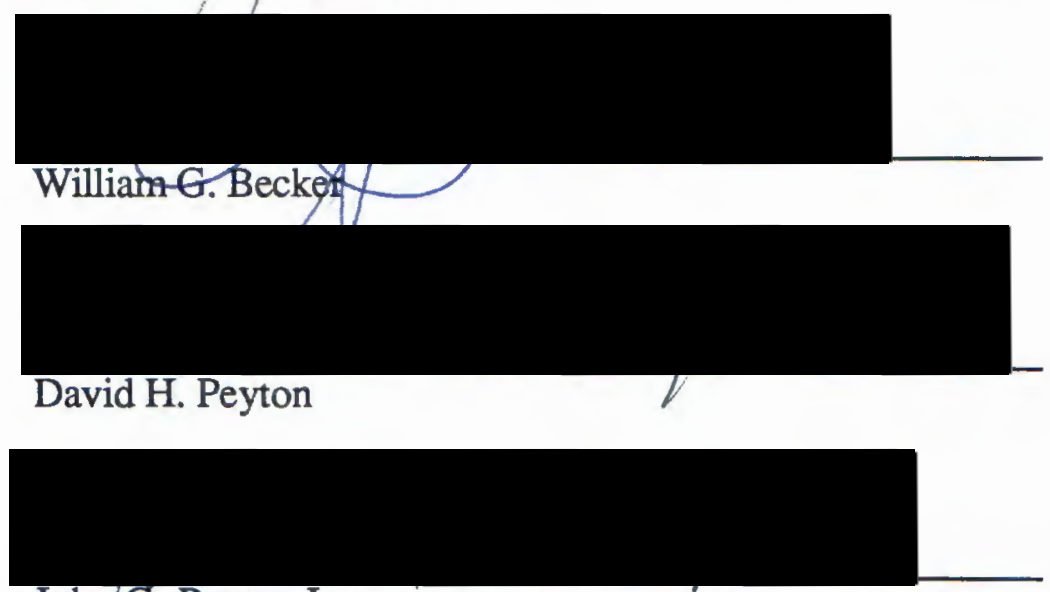
JohnlG. Rueter Jr.

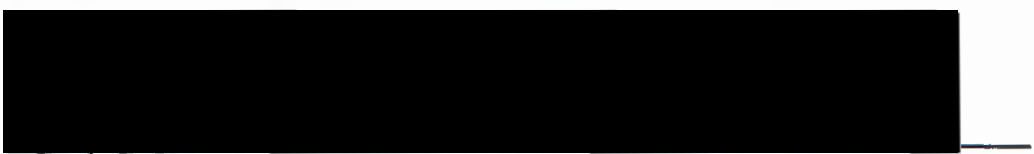

Carl C. Wamser

This study utilizes steady state fluorescence measurements, flash-induced P680+ absorption transients, and DCIP reduction kinetics to study the inhibitory effects of linolenic acid (LA) upon Photosystem II (PSII) in whole spinach chloroplasts and insideout wheat thylakoids. It confirms the presence within PSI of LA-induced inhibition of energy trapping and/or primary charge separation (i.e., primary inhibition), in addition to 
donor side inhibition. The latter is diminished in the presence of 1,5-Diphenylcarbohydrazide (DPC) and probably takes place at the oxygen evolving complex. Primary inhibition, which is more controversial, probably occurs between $\mathrm{Ph}$ and $\mathrm{Q}_{\mathrm{A}}$, with a likely contribution at the level of PSII energy trapping. In addition, the ability of $\mathrm{Mg}^{2+}$ to delay a drop in steady state fluorescence intensity normally associated with thylakoid exposure to LA is explained by the ability of this cation to confer resistance to LA-induced destacking of thylakoid membranes.

Steady state fluorescence results in the presence of DCMU, dithionite and LA also support the presence of an additional acceptor between $\mathrm{Ph}$ and $\mathrm{Q}_{\mathrm{A}}$. This acceptor, designated here as " $\Re$," is proposed not to be a sequential member of the transport chain, but may be accessible to it via $Q_{A}$ when the chain blocked, such as with DCMU. $R^{-}$is proposed to exert a coulombic effect upon $\mathrm{Ph}$, thereby affecting the degree of primary charge recombination. It may be related to one of the several acceptors already proposed by others and the need for more study is stressed in order to confirm or refute its existence. 
AN ANALYSIS OF THE INHIBITORY EFFECTS OF LINOLENIC ACID

UPON PHOTOSYSTEM II OF HIGHER PLANTS

by

MARK EDWARD IVEN

A thesis submitted in partial fulfillment of the requirements for the degree of

\section{MASTER OF SCIENCE \\ in \\ CHEMISTRY}

Portland State University

1989 
TO THE OFFICE OF GRADUATE STUDIES:

The members of the Committee approve the thesis of Mark Edward Iven presented February 2, 1989.

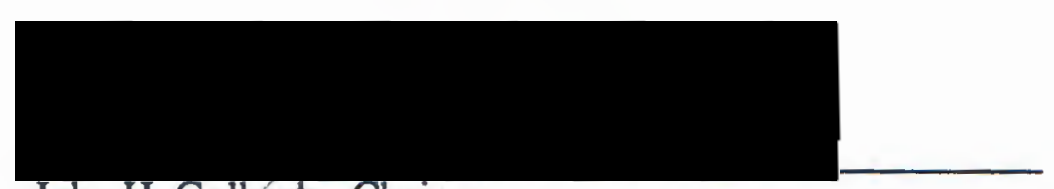

John H. Golbeok, Chair
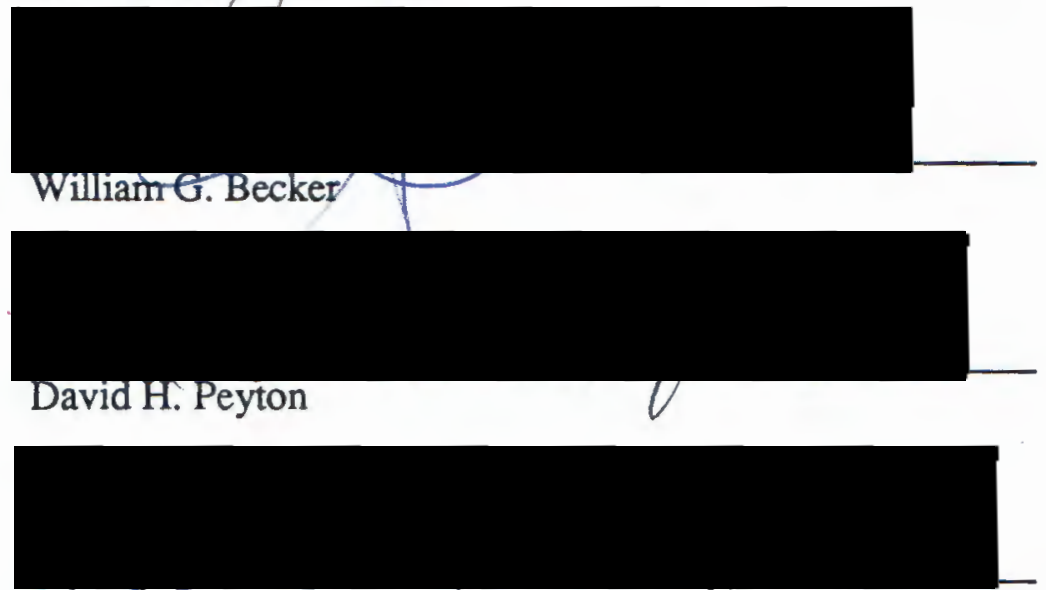

John 4. Rueter Jr.

Carl C. Wamser

\section{APPROVED:}

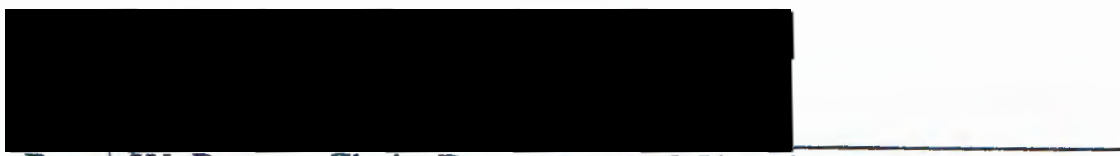

Bruce W. Brown, Chair, Department of Chemistry

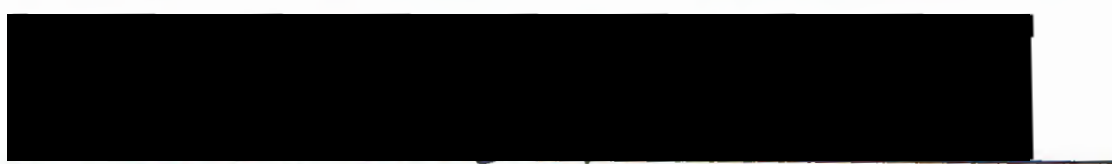

Bernard Ross, Vice Provost for Graduate Studies 


\section{ACKNOWLEDGEMENTS}

I wish to thank Dr. John Golbeck for his patient assistance during the course of this work. In addition, I would like to thank all the thesis committee members, and Kevin Parrett, for their valuable and timely comments during the manuscript phase of this thesis. I also would like to express appreciation to Chuck Haymond and to the science support services for their assistance in designing and building the log converter amplifier used in the DCIP kinetic experiments. Finally, I would like to thank those around me, both within the university and without, who offered many helpful suggestions and who were a constant source of encouragement. 


\section{TABLE OF CONTENTS}

PAGE

ACKNOWLEDGEMENTS.................................................

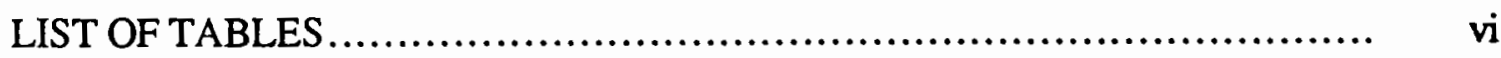

LIST OF FIGURES .................................................. vii

\section{CHAPTER}

I INTRODUCTION ........................................ 1

Inhibition of PSII....................................... 4

Herbicides

Linolenic Acid

Detection of Inhibition

Research Goal........................................... 7

II FLUORESCENCE ...................................... 9

Emission Wavelengths.................................. 10

Fluorescence Decay Kinetics........................... 12

Additional Components .............................. 15

Klimov Hypothesis......................................... 15

Alternative Views ................................... 16

Energy Distribution Models ............................... 18

III MATERIALS AND METHODS ............................. 21

Fluorescence Study....................................... 21

Chloroplast Isolation

Chemical Reagents

Apparatus 
Flash Photolysis

Photosystem II Particles

Chemical Reagents

Apparatus

Data Aquisition

DCIP Bleaching Kinetics

Chloroplast Prep

Chemical Reagents

Apparatus

IV DATA PRESENTATION..................................... 34

Fluorescence

DPC $(500 \mu \mathrm{M})$

Dithionite, $\mathrm{S}_{2} \mathrm{O}_{4}{ }^{2-}(3-4 \mathrm{mM})$

DCMU $(5 \mu \mathrm{M})$

DCIP Reduction

P680+ Absorption Transients............................... 42

V DISCUSSION............................................... 44

DCIP Reduction Kinetics................................... 44

Fluorescence .......................................... 46

Magnesium Effects

Mediator Effects

Model ................................................. 50

VI CONCLUSION ........................................... 54

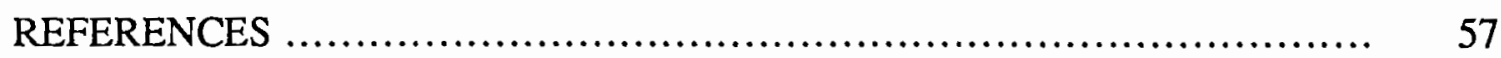

APPENDIX A ....................................................... 61

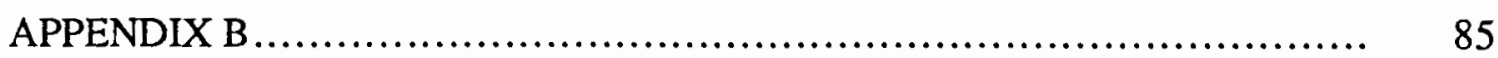




\section{LIST OF TABLES}

TABLE

PAGE

I Decay Components of the Fluorescence Kinetics for Spinach

Chloroplasts According to Haehnel et al........................ 12

II Suggested Relationship Between Decay Component Lifetimes at

Open and Closed PS II Reaction Centers Based on the

Concept of $\alpha, \beta$-Heterogeneity According to Holzwarth, et al. .

III Decay Components of the Fluorescence Kinetics for Spinach

Chloroplasts According to Tabbutt et al............................. 


\section{LIST OF FIGURES}

FIGURE

PAGE

1a. Schematic Representation of Photosystem II $\quad$......................... 2

1b. Photosystem II Energy Scheme for Plants....................................... 3

2. Spectrofluorometer Apparatus...................................... 24

3. P680 ${ }^{+}$Flash Photolysis Spectrophotometer.......................... 27

4. Spectrophotometer to Monitor DCIP Bleaching Kinetics ................ 30

5. Detector Circuitry for DCIP Bleaching Spectrophotometer.............. 32

6. Fluorescence Rise Curves for Whole Chloroplasts in the Presence of

$150 \mu \mathrm{M}$ Linolenic Acid................................................ 62

7. Fluorescence Rise Curves for Whole Chloroplasts in the Presence of

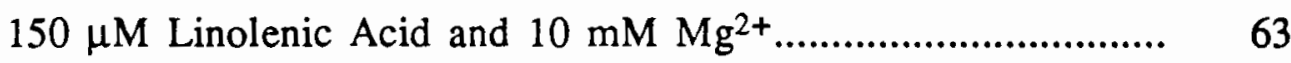

8. Relative Fluorescence Intensity vs. Linolenic Acid Concentration.......... 64

9. Fluorescence Rise Curves for Whole Chloroplasts in the Presence of

$67 \mu \mathrm{M}$ Linolenic Acid and $10 \mathrm{mM} \mathrm{Mg}^{2+}$

10. Fluorescence Rise Curves for Whole Chloroplasts at pH 6.0 (HEPES) in

the Presence of $150 \mu \mathrm{M}$ Linolenic Acid..................................... 66

11. Fluorescence Rise Curves for Whole Chloroplasts in the Presence of $5 \mu \mathrm{M}$

DCMU and $\mathrm{Mg}^{2+}$

12. Relative Fluorescence Intensity vs. Linolenic Acid Concentration in the

Presence of $500 \mu \mathrm{M} \mathrm{DPC}$

13. Fluorescence Intensity vs. Linolenic Acid Concentration in the Presence of $10 \mathrm{mM} \mathrm{Mg}^{2+},+/-\mathrm{DPC}$ 
14. Relative Fluorescence Intensity $v s$. Time of Incubation with $150 \mu \mathrm{M}$

Linolenic Acid, $+/-\mathrm{Mg}^{2+}$ 70

15. Relative Fluorescence Intensity $v s$. Incubation Time with Dithionite,

$+/$ - Linolenic Acid and $\mathrm{Mg}^{2+}$

16. Relative Fluorescence Intensity vs. Incubation Time with $150 \mu \mathrm{M}$

Linolenic Acid in the Presence of $20 \mathrm{mM} \mathrm{Mg}^{2+},+/$ - Dithionite.

17. Relative Fluorescence Intensity $v s$. Incubation Time with $5 \mu \mathrm{M}$ DCMU, $+/$ - Linolenic Acid and $\mathrm{Mg}^{2+}$

18. Relative Fluorescence Intensity $v s$. Incubation Time with $150 \mu \mathrm{M}$

Linolenic Acid and $20 \mathrm{mM} \mathrm{Mg}^{2+},+/$ - DCMU

19. Average DCIP Reduction Rates vs. Time for Differing Concentrations of Linolenic Acid, +/- DPC

20. Average DCIP Reduction Rates vs. Linolenic Acid Concentration (10 sec

Incubation) at Differing Excitation Light Intensities, +/- DPC

21. Direct Plots According to the Method of Cornish-Bowden of DCIP

Reduction Rates vs. $650 \mathrm{~nm}$ Excitation Light Intensity

22. Average DCIP Reduction Rates vs. Linolenic Acid Concentration (5 min Incubation) at Differing Excitation Light Intensities, +/- DPC 78

23. DCIP Reduction Rates vs. $650 \mathrm{~nm}$ Excitation Light Intensity at Differing Concentrations of Linolenic Acid (10 sec Incubation), +/- DPC ........

24. DCIP Reduction Rates vs. $650 \mathrm{~nm}$ Excitation Light Intensity at Differing Concentrations of Linolenic Acid (5 min Incubation), +/- DPC 80

25. Direct Plots According to the Method of Cornish-Bowden of DCIP Hill Reaction Rates vs. $650 \mathrm{~nm}$ Excitation Light Intensity 
26. Re-reduction Kinetics of Photooxidized P680+ at pH 7.6 (HEPES) ........

27. Observed $\mathrm{P} 680^{+}$Concentration vs. Time at Various $\mathrm{pH}$ in the Presence of 


\section{CHAPTER I}

\section{INTRODUCTION}

Photosynthesis involves the conversion of light energy into chemical energy. The process in plants and algae obeys the overall net reaction

$$
6 \mathrm{CO}_{2}+6 \mathrm{H}_{2} \mathrm{O} \longrightarrow \mathrm{C}_{6} \mathrm{H}_{12} \mathrm{O}_{6}+6 \mathrm{O}_{2}
$$

with a standard free energy change of $+686 \mathrm{kcal} / \mathrm{mol}(+2870 \mathrm{~kJ} / \mathrm{mol})$. This reaction occurs in two separate stages: a photochemical phase and an enzymatic dark phase. In higher plants, the photochemical or "light" reactions, so called because they occur only in light, take place in thylakoid membranes located within chloroplasts according to the equation:

$$
2 \mathrm{H}_{2} \mathrm{O}+2 \mathrm{NADP}^{+}+8 \mathrm{hv} \longrightarrow 2 \mathrm{NADPH}+\mathrm{O}_{2}+2 \mathrm{H}^{+}
$$

This reaction involves the net reduction of $\mathrm{NADP}^{+}$at the expense of water. It also involves the phosphorylation of ADP to form ATP (not shown) by utilizing a free energy gradient produced in part as protons generated from (2) accumulate on the inner side of the thylakoid membrane. The enzymes in the dark reactions are not membrane-bound, but require the products formed in the light reactions in addition to carbon dioxide:

$$
\begin{array}{r}
6 \mathrm{CO}_{2}+18 \mathrm{ATP}+11 \mathrm{H}_{2} \mathrm{O}+12 \mathrm{NADPH}+12 \mathrm{H}^{+} \longrightarrow \\
\mathrm{F}-6-\mathrm{P}+17 \mathrm{P}_{i}+18 \mathrm{ADP}+12 \mathrm{NADP}^{+}
\end{array}
$$


where F-6-P is an abbreviation for fructose-6-phosphate. Equation (3) depicts the net reaction of the Calvin cycle in which the seven-carbon intermediate D-ribulose-1,5bisphosphate is first used and then regenerated in a cycle that utilizes various triose phosphates as intermediates. The ADP and NADP+ are circulated back to the thylakoid membrane and then reincorporated into the light reactions. Plants use the F-6-P produced to synthesize the starch, cellulose and sucrose necessary for existence.

Absorption of light occurs sequentially at two membrane-bound complexes, termed photosystems I (PSI) and II (PSII). A schematic diagram of the PSII complex is shown in Fig. 1a.

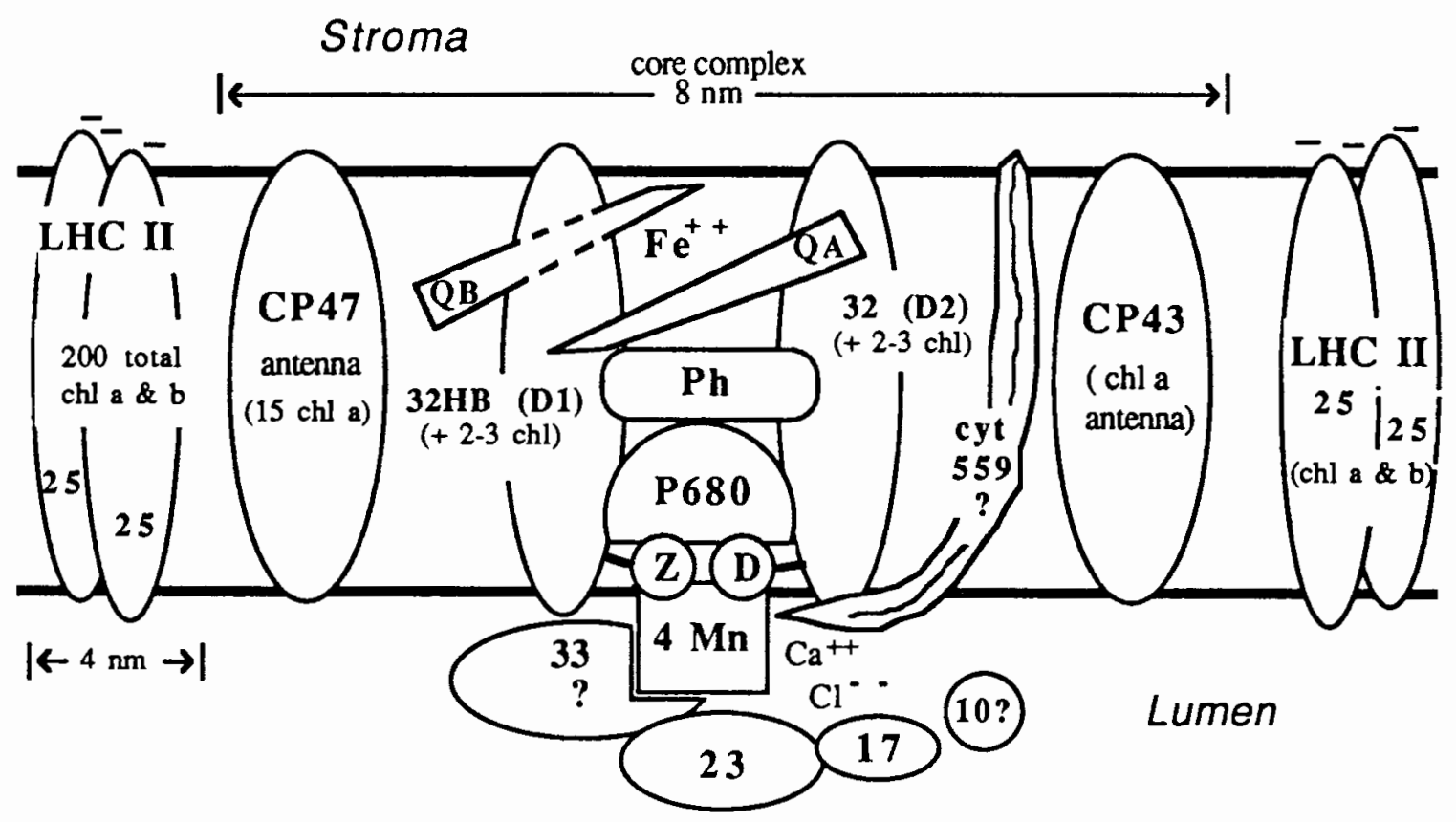

Figure 1a. Schematic representation of Photosystem II. Bold numbers, with the exception of Cyt 559 and P680, refer to the approximate size in kilodaltons ( = MW / 1000) of individual polypeptides. Not to scale.

This study is concerned specifically with the light reactions of PSII. A useful representation of the light reaction phase is the classic "Z-scheme," which relates the various components of PSI and PS II to each other on a midpoint potential scale. That portion of the Z-scheme dealing with PSII is shown in Fig. $1 \mathrm{~b}$. 


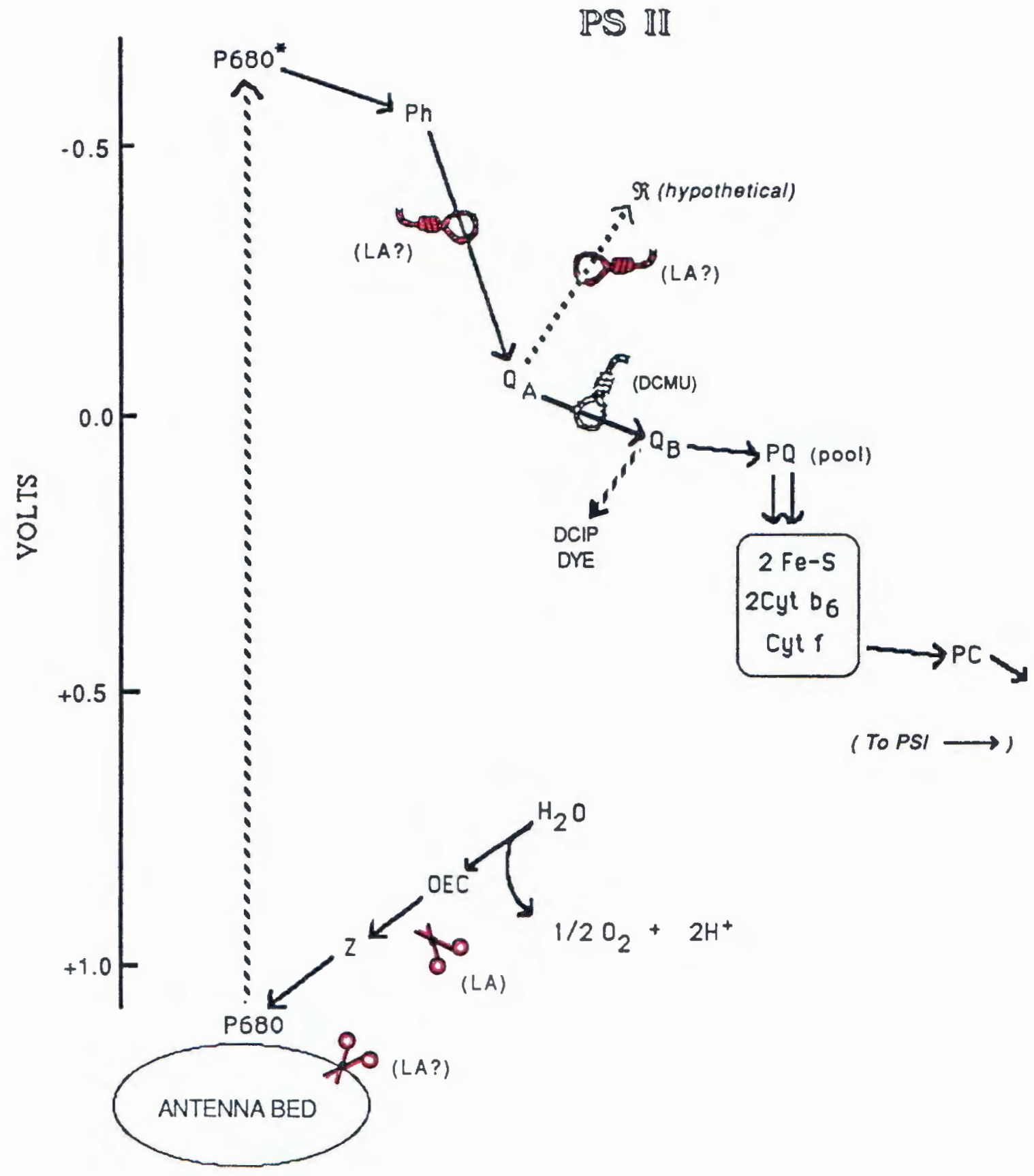

Figure 1b. Photosystem II Energy Scheme for Plants. Red symbols represent either known or proposed sites of inhibition by linolenic acid. 
Energy transfer to $\mathrm{P} 680$ forms the powerful reductant $\mathrm{P} 680^{*}\left(\mathrm{E}_{\mathrm{m}}<-0.600 \mathrm{mV}\right)$, which reduces pheophytin $(\mathrm{Ph})$ within a few picoseconds in the primary charge separation step.of PSII. This charge separation is stabilized across the thylakoid membrane as $\mathrm{Ph}^{-}$ reduces the plastoquinone $Q_{A}\left(E_{m}=-130 \mathrm{mV}\right)$ in about 100 psec. $Q_{A}$ then reduces the plastoquinone $\mathrm{Q}_{B}\left(\mathrm{E}_{\mathrm{m}}=+100 \mathrm{mV}\right)$ much more slowly, in about $100 \mu \mathrm{sec}$. Once $\mathrm{Q}_{B}$ has been doubly reduced it is released from its binding site on polypeptide D1 and into a pool of mobile plastoquinones (10-20 molecules per $P 680)$. $Q_{B}$ is eventually replaced on D1 with another quinone from the pool. Reduced hydroquinone from the pool reduces the cyt $\mathrm{b}_{6} / \mathrm{f}$ complex in the rate-limiting step of PSII $\left(\mathrm{t}_{1 / 2}=15-20 \mathrm{msec}\right)$, and cytochrome $\mathrm{f}$ within the complex in turn reduces the mobile protein plastocyanin (PC) on the inner side of the thylakoid membrane. It is $\mathrm{PC}$ which goes on to transfer reducing equivalents formed in PSII on to PSI (not shown).

The $\mathrm{P} 680^{+}$formed during primary charge separation has a midpoint potential of $\approx+1.3 \mathrm{~V}-$ a sufficiently strong oxidant to severely damage the photosystem unless quickly neutralized. This neutralization step occurs typically within nanoseconds as electrons are transferred from oxidized water to $\mathrm{P}_{680^{+}}$via an $\mathrm{O}_{2}$-evolving protein complex (OEC) containing an ensemble of four molecules of manganese. Actual donation of electrons from the OEC to $\mathrm{P} 680^{+}$occurs by a component designated " $\mathrm{Z}$," which has recently been identified as a redox active tyrosine radical located on D1 (Fig. 1a) [Debus et al., 1988]. " $\mathrm{D}$ " is also a tyrosine residue located on D2 and which is able to donate slowly to $\mathrm{P} 680^{+}$when $\mathrm{Z}$ is disabled [Debus et al., 1988]. The physiological role of $\mathrm{D}$ is uncertain.

\section{Inhibition of PSII}

Herbicides. Many compounds are known to interact with the electron transport chain of PSII. The herbicides monuron (3-(3'-monochlorophenyl)-1,1-dimethylurea, 
abbreviated as CMU) and diuron (3-(3',4'-dichlorophenyl)-1,1-dimethylurea, abbreviated as $D C M U$ ), for example, kill plants by efficiently binding to the $Q_{B}$ site on $D 1$, effectively blocking electron transport between $\mathrm{Q}_{\mathrm{A}}$ and $\mathrm{Q}_{B}$ (Fig. 1 b). Atrazine and other triazine herbicides function in a similar manner. Structural similarities relating the physiological activity of these two types of compounds include a carbonyl or equivalent group and a positively charged nitrogen in each [Trebst, 1987].

Linolenic Acid. It has long been known that fatty acids, especially unsaturated fatty acids, inhibit photosynthesis when added to functioning systems [Spikes et al., 1955][Krogmann and Jagendorf, 1959]. This may seem surprising considering that about $30 \%$ of the total thylakoid mass of higher plants is composed of esterified acyl lipids, with polyunsaturated fatty acids constituting nearly $90 \%$ of these [Murphy, 1986]. Linolenic acid (LA), a trienoic $\mathrm{C}_{18}$ fatty acid with structure $\mathrm{CH}_{3}\left(\mathrm{CH}_{2} \mathrm{CH}=\mathrm{CH}\right)_{3}\left(\mathrm{CH}_{2}\right)_{7} \mathrm{COOH}$, comprises $72 \%$ of the total thylakoid acyl lipid fraction in chloroplasts. The inhibitory effects of exogenous LA on the photosynthetic apparatus in chloroplasts are multiple, but are known to affect mainly PSII. Although progress has been made, the predominant mode of inhibition by LA within PSI appears to be unique and has yet to be identified. This forms the basis for the present work.

To date linolenic acid has been reported to: i.) participate in the disruption of chloroplast and thylakoid membrane structure [Cohen et al., 1969][Shaw et al., 1976][Okamoto et al., 1977] ii.) inhibit the donor complex by various means [Siegenthaler, 1974][Golbeck et al., 1980][Venediktov and Krivoshejeva, 1983][Golbeck and Warden, 1984][Warden and Csatorday, 1987][Garstka and Kaniuga, 1988] iii.) stimulate electron flow due to uncoupling of phosphorylation [Cohen et al., 1969][Okamoto and Katoh,1977][Golbeck et al., 1980], iv.) cause primary inhibition, either by affecting the stability of primary charge separation or by preventing its occurrence, or a combination of both [Golbeck et al., 1980][Vernotte et al., 1983][Warden 
and Csatorday, 1987].

Detection of Inhibition. The blue dye DCIP (2,6-dichlorophenol indophenol) has a midpoint potential of $220 \mathrm{mV}(\mathrm{pH} 7)$ which enables it to replace $Q_{B}\left(E_{m}=+100 \mathrm{mV}\right)$ as an electron acceptor from $Q_{A}$, thus effectively disabling the chain at that point (Fig. $1 b$ ). DCIP bleaches when reduced, presenting a handy tool for monitoring the viability of the PSII reaction center. For example, inhibition of the transport chain between $Q_{A}$ and $Q_{B}$ or between $\mathrm{Ph}$ and $\mathrm{Q}_{\mathrm{A}}$ would be expected to stop or slow the normal ability of PSII to bleach DCIP. Similarly, an inhibition of the processes leading to primary charge separation should also exhibit the same effect under the same experimental conditions. If the latter process involved a partial dissociation of the large light-gathering antenna bed (which includes both the LHC II and actual "antenna" units - see Fig. 1a), then an increase in light intensity might restore the electron flow rate through the chain to a normal value as remaining pigments are utilized to a greater degree. However, inhibition of the chain itself, as in the former example, would not be expected to be overcome merely by employing a higher light intensity since there is no useful alternative path for electrons. This type of kinetic analysis will be used in the current study in an attempt to identify the type(s) of inhibition associated with LA.

Not only can PSII inhibition be monitored using DCIP absorption kinetics, but fluorescence emission is also a valuable tool. Although chlorophyll is a strong fluorophore, the chlorophyll bed associated with functioning PSII reaction centers fluoresces very little at low exitation intensities as its energy is funneled quickly into the reaction center and translated into photochemistry. At higher light intensities, or upon the addition of inhibitors such as DCMU, fluorescence emission from the bed increases significantly. Therefore, high fluorescence intensity may indicate either a damaged (or inhibited) PSII center or a normal center whose rate of photochemistry is simply unable to match the rate of energy absorption in bright light. Fluorescence emission may thereby act 
as a safety mechanism to release excess energy without the production of damaging heat or side reactions in vivo.

Emission intensity has been correlated to the steady-state concentration of reduced $\mathrm{Q}_{\mathrm{A}}$ [Van Gorkom, 1974]. High excitation light intensities and/or addition of DCMU cause an accumulation of $\mathrm{Q}_{\mathrm{A}}{ }^{-}$(Fig. 1b). The actual mechanism of in vivo fluorescence emission is controversial, however. Some, such as Holzwarth et al. [1985], believe that the energy aquired in inhibited PSII centers is emitted immediately from the light-harvesting apparatus, whereas others, such as Klimov et al.[1977, 78], believe that absorbed energy first leads to primary charge separation and then to recombination of $\mathrm{Ph}^{-}$and $\mathrm{P}^{6} 80^{+}$prior to being released into the antennae bed for emission.

In addition to herbicides such as DCMU, exogenous linolenic acid is also associated with a high fluorescence emission. This emission has different properties than that associated with other inhibitors, however, as this study will explore. The inhibitory effects of LA may seem surprising considering its role as the predominant polyunsaturated lipid present in the higher plant membrane. The fact that free LA is not present in appreciable amounts in healthy plants implies that it is the free and not the esterified form of the fatty acid which acts as an inhibitor. The reason for this is uncertain but may be rationalized as part of a mechanism to protect the plant under conditions where the donor system has been damaged and is unable to function. This might signal the plant to begin hydrolyzing membrane lipids, with the free LA thus liberated shutting down PSII to prevent any further damage by a potential accumulation of $\mathrm{P} 680^{+}$. Absorbed energy would then be released harmlessly as fluorescence.

\section{$\underline{\text { Research Goal }}$}

This study utilizes steady state fluorescence measurements, flash-induced P680+ absorption transients, and DCIP reduction kinetics to study the inhibitory effects of LA 
upon PSII in whole spinach chloroplasts and inside-out wheat thylakoids. It confirms the presence within PSII of LA-induced inhibition of energy trapping and/or primary charge separation (i.e., primary inhibition), in addition to donor side inhibition. Primary inhibition, which is the more controversial type, is concluded to occur between $\mathrm{Ph}$ and $Q_{A}$, with the ensuing increase in fluorescence intensity preceded by primary charge separation / recombination. In addition to inhibition of the chain, a likely contribution to the inhibition process is concluded to occur at the level of PSII energy trapping. The latter implies an interference with primary charge separation and may be a consequence of the LA-induced dissociation of a portion of the light-gathering antenna bed from the reaction center.

Steady state fluorescence results in the presence of DCMU, dithionite and LA also support the presence of an additional acceptor between $\mathrm{Ph}$ and $\mathrm{Q}_{\mathrm{A}}$. This acceptor, designated here as $\Re$, is proposed not to be a sequential member of the transport chain but may be accessible to it via $Q_{A}$ when the chain blocked, such as with DCMU. $\Re^{-}$is proposed to exert a coulombic effect upon $\mathrm{Ph}$, thereby affecting the degree of primary charge recombination. It may be related to one of the several acceptors already proposed by others and the need for more study is stressed in order to confirm or refute its existence.

The complexity of this goal requires incorporation of the results of other researchers, particularly in the area of fluorescence, in order to construct a viable model of the LA inhibition process. The following chapter further explores the sources and characteristics of fluorescence from photosynthetic units and attempts to clarify some of the controversy surrounding this subject. 


\section{CHAPTER II}

\section{FLUORESCENCE}

Ever since Strehler and Arnold [1951] observed light emission from photosynthetic algae nearly forty years ago, fluorescence measurements have been widely used to probe the light reactions of photosynthesis. For example, such a probe has been used to determine the midpoint potential $\left(\mathrm{E}_{\mathrm{m}}\right)$ and redox state of various PSII acceptors [Cramer and Butler, 1969][Ke et al., 1976][Golbeck and Kok, 1979]. In general, fluorescence offers a sensitive and non-destructive method of monitoring the emitting excited states of various pigments in vivo, including chlorophyll. It has been widely used as an indirect monitor of the functional state of the PSII reaction center. Despite its usefulness, however, fluorescence has been accompanied by much controversy centered around disagreement over the sources of the multiple emission and quenching components. The recent use of single photon timing (SPT) techniques utilizing pulsed lasers has enabled researchers to resolve many of these components with lifetime decays in the picosecond time frame. This has been valuable for the study of photosynthesis because it involves several primary energy transfer steps which occur within this time frame. Much confusion still exists, however, and since a fluorescent probe is used in the present investigation, a brief summary of the state of knowledge in this subject area is appropriate.

Total chlorophyll fluorescence emission from any active photosynthetic apparatus depends especially upon the state of PSII. When PSII is fully "open," i.e., when all secondary electron acceptors remain photoactive, the system emits at its lowest intensity, termed $\mathrm{F}_{o}$. At the other extreme, when normal photochemistry has been terminated (PSII 
fully "closed"), emission intensity is at a maximum $\left(\mathrm{F}_{\max }\right)$. The latter state can be achieved by reducing all the secondary acceptors either chemically or with light, or it can be hastened by isolating the PSII electron transport chain at the appropriate location using chemical inhibitors such as DCMU (3-(3',4'-dichlorophenyl)-1,1-dimethylurea). One can easily observe the fluorescence emission from normal dark-adapted chloroplasts increase from $\mathrm{F}_{o}$ to $\mathrm{F}_{\max }$ upon illumination, a process termed "induction." The variable portion between the two extremes is called $F_{v a r}$ (or $\Delta F$ ) and is polyphasic [Neubauer and Schreiber, 1987] [Schreiber and Neubauer, 1987]. The overall variable event for untreated chloroplasts takes typically 3 seconds or less, depending on factors such as excitation light intensity, and was identified quite early in photosynthesis research. It was linked eventually to the redox state of an early unidentified acceptor, deemed "Q" for "quencher" [Delosme, 1967]. Conveniently, Q was determined later to be a quinone specifically " $\mathrm{Q}_{A}$," the first of two (the other quinone is designated $\mathrm{Q}_{B}$ ) [Van Gorkom, 1974]. Much evidence of quinone-acceptor heterogeneities (other than $Q_{A} \& Q_{B}$ ) has been reported, based in large part upon fluorescence data. This has led, among other things, to proposals that either there are electron acceptors in addition to $Q_{A}$ [Evans et al. 1985] or that $Q_{A}$ itself may exist in multiple forms which may be associated with different types of PSII reaction centers (" $\alpha \& \beta$-centers") [Black et al., 1986][Anderson, 1987].

\section{Emission Wavelengths}

Fluorescence emission from chloroplasts and algae at room temperature is characterized by a single very broad band centered near $685 \mathrm{~nm}$ [Cho and Govindgee, 1970]. At $77 \mathrm{~K}$ this $\mathrm{F}_{685}$ band becomes more pronounced and is accompanied by longer wavelength emission between 710 and $740 \mathrm{~nm}$ [Breton, 1982]. The latter band was not observed by van Dorssen et al. [1987] in oxygen-evolving PSII core particles devoid of LHC II. A well known shoulder at $695 \mathrm{~nm}$ also appears at $77 \mathrm{~K}$ and greatly increases at 
lower temperatures, eventually becoming the predominant band at $4 \mathrm{~K}$ [van Dorssen et al., 1987]. Interestingly, the $695 \mathrm{~nm}$ band was specifically diminished in dithionite-treated samples which were pre-illuminated prior to freezing at $77 \mathrm{~K}$, whereas similar samples without pre-illumination exhibited normal $\mathrm{F}_{695}$ intensity [Renger et al., 1983]. Data on an $\mathrm{F}_{680}$ band is more difficult to interpret due to conflicting results by different groups. Moya and Garcia [1983] have applied a unique method of deconvoluting emission spectra at $77 \mathrm{~K}$ into seven distinct Gaussian bands. This method depicts the $\mathrm{F}_{720}-\mathrm{F}_{740}$ region as being composed chiefly of two bands with peaks at $714 \mathrm{~nm}$, designated B3, and at 724 $\mathrm{nm}$, designated B4. Both are ascribed to PSI emission at this low temperature, which is consistent with the observed lack of these bands in PSII particles [van Dorssen et al., 1987]. The authors label $F_{685}$ as $B 1$ and describe it as being composed of two smaller peaks: B'1, attributed to PSII antennae, and B"1, attributed either to chlorophyll which has become disassociated from a reaction center, or to delayed luminescence from PSII antennae resulting from primary charge recombination within the reaction center. The latter possibility is consistent with a model suggested by Klimov et al. [1978] and is discussed below along with contrasting models. The authors also depict $\mathrm{F}_{695}$ as being comprised of two bands, designated $\mathrm{B}^{\prime} 2$ and $\mathrm{B}^{\prime 2} 2$, which are analogous to $\mathrm{B}^{\prime} 1$ and $\mathrm{B}^{\prime \prime} 1$ and which have similar origins. Breton used polarized light spectroscopy to conclude that at low temperatures Klimov-like charge recombination energy may excite either P680 or pheophytin, leading to $F_{685}$ and $F_{695}$, respectively [Breton, 1982, 83]. This was affirmed recently by van Dorssen et al. [1987]. Holzwarth [1986], a prominent opponent of the Klimov model, agrees that one may associate $\mathrm{F}_{695}$ with pheophytin provided that $\mathrm{Ph}^{*}$ is generated by energy transfer directly from excited antenna chlorophyll, thus obviating the requirement of an intervening primary charge separation step. 
Fluorescence Decay Kinetics

Recent advances in the single-photon timing (SPT) technique have provided a method for measuring fluorescence decays in the picosecond time range with unsurpassed sensitivity. Unfortunately, results using this method have been varied and often cryptic, leading frequently to contradictory conclusions. The technique remains a valuable one, however, and will undoubtedly become more so as refinements are developed.

An early use of SPT on various species of plants and algae identified at least three decay components whose lifetimes and yields varied depending on whether measurements are made at $F_{o}$ or $F_{\max }$. Typical values reported by Haehnel for spinach are listed in the following table:

\section{TABLE I}

DECAY COMPONENTS OF THE FLUORESCENCE KINETICS FOR SPINACH CHLOROPLASTS ACCORDING TO HAEHNEL ET AL.

$\begin{array}{lcccccc} & \tau_{1}(\mathrm{psec}) & \phi_{1} & \tau_{2} & \phi_{2} & \tau_{3} & \phi_{3} \\ & & & & & & \\ \mathrm{~F}_{o} & 110 & 10 & 420 & 78 & 1200 & 12 \\ \mathrm{~F}_{\max } & 50 & 4 & 750 & 68 & 2000 & 330\end{array}$

(Taken from Haehnel et al., 1982, p. 168). $\phi_{\mathrm{i}}$, the relative fluorescence yield of the $\mathrm{i}^{\text {th }}$ component, is calculated by: $\phi_{i}=\alpha_{i} \tau_{i} / \Sigma \alpha_{i} \tau_{i}$, where $\alpha_{i}$ is the initial intensity and $\tau_{i}$ is the lifetime of the $\mathrm{i}^{\text {th }}$ component, respectively. The yields listed are normalized to the total yield of $F_{0}(\equiv 100)$.

Additionally, Haehnel has reported that the fast decay component for Chlorella vulgaris and Chlamydomonas reinhardtii has a lifetime of approximately $120 \rrbracket 80 \mathrm{psec}\left(" \tau_{f}\right)$ and a yield of $17 \% \rrbracket 2 \%$ for $F_{o} \backslash \mathrm{F}_{\max }$ [Haehnel et al., 1983]. He concluded that this component probably is caused by the quenching of core antenna chl $a$ by the open PSII reaction center since its yield decreases nearly to zero as the center is closed. He further 
concluded that this component (and, therefore, at least $17 \%$ of $\mathrm{F}_{o}$ ) does not directly result from any particular photochemical event within the reaction center and that the contribution of PSII to $\tau_{f}$ disappears at $F_{\max }$. Holzwarth has suggested that $\tau_{f}$ may actually be composed of two components, based upon both inhibitor effects and the dependence of this component upon excitation wavelength - although he was cautious enough to point out the uncertainties inherent in the mathematically intricate SPT fitting procedure. He assigned the slower component ( 180 psec) to open PSII centers, possibly $\alpha$-centers only, and the faster ( $80 \mathrm{psec}$ ) to PSI. He suggested that the $180 \mathrm{psec}$ component is variable and turns into the $2.2 \mathrm{nsec}$ long-lived component (discussed below) upon PSII center closure (Table II) [Holzwarth et al., 1985].

\section{TABLE II}

\section{SUGGESTED RELATIONSHIP BETWEEN DECAY COMPONENT LIFETIMES AT OPEN AND CLOSED PSII REACTION CENTERS BASED ON THE CONCEPT OF $\alpha, \beta$-HETEROGENEITY ACCORDING TO HOLZWARTH, ET AL. (for Chlorella vulgaris)}

$\begin{array}{ccc} & \begin{array}{c}\text { Open PSII } \\ \text { reaction center }\end{array} & \begin{array}{c}\text { Closed PSII } \\ \text { reaction center }\end{array} \\ \text { PSII( } \alpha) & 180 \text { psec } & 2200-2400 \text { psec } \\ \text { PSII( } \beta) & 500-600 & 1200-1400 \\ \text { PSI } & 80 & 80\end{array}$

(Taken from Holzwarth et al., 1985, p.165)

Hodges and Moya [1987] have also detected a variable fast component in PS II particles whose lifetime changes from 20 to $250 \mathrm{psec}$ as centers become closed. Therefore, in contrast to the Holzwarth group, they have reported the existence of a significant fast phase (250 psec) from PSII even at $F_{\max }$. 
Haehnel found that $\tau_{m}$, the $500-600$ psec middle component, is nearly constant with a yield comprising nearly all of the remaining $F_{o}$ [Haehnel et al., 1983]. This implies that $\mathrm{F}_{o}$ is comprised essentially of two invariable components, $\tau_{f}$ and $\tau_{\mathrm{m}}$, whose lifetimes are independent of the state of the PSII reaction center. He attributed the origin of $\tau_{m}$ to energy transfer from LHC II to the chl $a$ antenna chlorophyll, citing both its lifetime increase by $<2$ when centers are closed - unlikely if from more closely-coupled antenna and its fluorescence maximum at $685 \mathrm{~nm}$, which is typical for LHC II chl $a / b$ proteins. He also pointed out that a nearly constant contribution of LHC II emission at both $\mathrm{F}_{o}$ and $F_{\max }$ could explain the $\mathrm{F}_{\text {max }} / \mathrm{F}_{o}$ total yield ratio of 3-5, lower than predicted [Kamen, 1963] [Haehnel et al., 1982]. This point will be explored further below. Others have concluded that $\tau_{m}$ is variable, i.e., a part of $\mathrm{F}_{\text {var }}$ [Holzwarth et al., 1985][Moya et al., 1986][Hodges and Moya, 1987]. For example, Holzwarth reported the lengthening of $\tau_{m}$ into $1200-1400$ psec "middle" phase at $F_{\max }$ (Table II). He concluded that a large portion of the middle component is not related to LHC II based upon the emission and excitation spectra of this component. He attributed the origin of the $685 \mathrm{~nm}$ emission to chl $a$, thereby implicating the PSII antenna as the predominant source of the $\tau_{m}$ component. He also suggested that this component originates from $\beta$-centers only. Hodges and Moya [1987] suggested non- $\alpha / \beta$ type PSII heterogeneity involving alternate but interconnected LHC II populations to explain all of their variable phases. Thus, the origin of the middle phase and its decay is currently unresolved.

The slow 1.3-2.4 nsec $\tau_{s}$ component is acknowledged generally to be strongly related to $\mathrm{F}_{v a r}$ because its yield has been shown to increase greatly at $\mathrm{F}_{\max } v s . \mathrm{F}_{o}$ (Tables $I$ \& II) [Klimov et al., 1977][Haehnel et al., 1982,1983][Holzwarth et al., 1985]. Its emission maximum is at approx. $685 \mathrm{~nm}$. The simultaneous increase in both the yield and lifetime of this component as the RC is closed is considered by some to be good evidence of coupling between different types of antenna units and/or PSII centers [Haehnel et al., 
1983][Hodges and Moya, 1987]. More uncertain, however, is whether or not emission is the result of charge recombination luminescence as promulgated by Klimov. This point notwithstanding, Holzwarth points out that there is general agreement that the yield of the slow component reflects the percentage of closed PSI centers present and as such "should nearly disappear in the $\mathrm{F}_{o}$ state" [Holzwarth, 1986].

Additional Components. It should be stated that the three component decay approach may be over-simplified. Holzwarth et al. [1985] has used SPT analysis to suggest the possible existence of a four component fluorescence decay. He noted that the fast component could be broken down into two separate components. Nevertheless, he found that three components remained sufficient to describe the majority of the data. Hodges and Moya [1986] have also detected four phases. They pointed out that there is little variation in the deconvoluted components' lifetimes $v s$. emission wavelength when four components were assumed. When three components were assumed, however, each exhibited a lifetime minimum around $720 \mathrm{~nm}$, with the faster component lifetimes decreasing the most - up to $60 \%$ between $670 \mathrm{~nm}$ and $720 \mathrm{~nm}$. Schatz et al. [1987] found only two fluorescence decay components with $F_{o} \backslash F_{\max }$ lifetime ratios of $80 \backslash 520$ and $220 \ 1300$ psec. Although he detected one and possibly two additional longer-lived components, he attributed them to contributions by unavoidable sample impurities such as allophycocyanin.

\section{Klimov Hypothesis}

Klimov et al. [1977, 78] was the first to offer experimental evidence suggesting that $F_{v a r}$ originates from primary charge recombination luminescence. He accomplished this by using difference spectra from pea chloroplasts and PS II fragments to correlate the development of a positive broad peak at $450 \mathrm{~nm}$ ( $\mathrm{Ph}^{-*}$ formation) and negative peaks at $518,545 \& 685 \mathrm{~nm}(\mathrm{Ph})$ to quenching of a long-lived (2-4 nsec) fluorescence component. 
He postulated that when $\mathrm{Q}$ is in the photoactive unreduced state, the middle phase is quenched as energy is transferred quickly from $\mathrm{Ph}^{-*}$ onto $\mathrm{Q} .{ }^{1}$ As $\mathrm{Q}$ becomes reduced, however, the biradical $\mathrm{P} 680^{+\cdot} \mathrm{Ph}^{-\cdot}$ is forced to recombine, re-exciting P680 and presumably forming the emitting state $\mathrm{P} 680^{*} \mathrm{Ph} \mathrm{Q}^{-}$. According to Breton, this "delayed emission" could occur either from P680* or from excited core antenna [Breton, 1983]. Klimov further proposed that quenching of the slow component is attributable to the longlived state $\mathrm{P} 680 \mathrm{Ph}^{-\cdot} \mathrm{Q}^{-}$, basing this on an observed decline in $\mathrm{F}_{\text {var }}$ accompanying what he concluded was trapping of $\mathrm{Ph}^{-\cdot}$ via the photoreduction of $\mathrm{P} 680^{+\cdot}$ in preparations with intact donor systems. He calculated a $0.04-0.08 \mathrm{eV}$ activation energy for formation and recombination of the state $\mathrm{P} 680^{+\cdot} \mathrm{Ph}^{-\cdot}$ from Arrhenius plots of $\Delta \mathrm{F}_{685}\left(\Delta \mathrm{F} \equiv \mathrm{F}_{v a r}\right.$ $=\mathrm{F}_{\max }-\mathrm{F}_{o}$ ). Klimov's model is consistent with the potential dependence of $\mathrm{F}_{\max }$ found by Warden \& Csatorday upon titrating the $\mathrm{Ph} / \mathrm{Ph}^{-}$pair in centers closed with linolenic acid [Warden \& Csatorday, 1987]. Warden pointed out that if primary photochemistry in the fatty acid-inhibited $\mathrm{RC}$ had been destroyed then $\mathrm{F}_{\max }$ should be independent of ambient potential.

Alternative Views. The Klimov model for the source of $F_{v a r}$ and the slow decay component has been questioned by some groups. Some prefer a simpler Stern Volmertype deactivation scheme such as one described by Butler and Strasser [1977]. This scheme avoids invoking delayed luminescence following charge-recombination; instead, it describes the excitation and subsequent deactivation of PSII antenna, LHC II and P680* via parallel pathways, where the lifetime of the excited singlet state of the antenna chlorophyll is inversely proportional to the competing rate constant of RC photochemistry. More will be said about Butler's models below.

Most challenges to the Klimov model question its implication that charge separation occurs equally well in both closed and open centers. For example, Schatz et al.

1 Klimov was not yet aware of the existence of the two separate quinones, $Q_{A}$ and $Q_{B}$. 
[1987] has recently used picosecond absorbance changes to report a decrease in yield of $\mathrm{P} 680^{+} \mathrm{Ph}^{-}$upon reduction of $\mathrm{Q}_{A}$ with sodium dithionite, accompanied by an increased lifetime of $\mathrm{chl}^{*}$. The author thus concludes that trapping and charge separation are inhibited by $50-70 \%$ in closed centers and that most of the emission at $F_{\max }$ is therefore "prompt" fluorescence from antenna $\mathrm{chl}^{*}$ and not "delayed" luminescence. He also reported a diminution of the $\tau_{f}$ yield by about $30 \%$ upon the closure of PSII centers, a lesser extent than that found in the earlier study by Haehnel et al. [1983] who reported a near disappearance of $\tau_{f}$ upon RC closure. Although the disappearance of $\tau_{f}$ has not been observed by other groups to date, its reduction by $30 \%$ is no less inconsistent with the Klimov model if this component indeed arises from the quenching of the antenna chlorophyll by $\mathrm{P} 680^{+}$as both Schatz and Haehnel assert. Instead, the Klimov model predicts that its yield should mirror the trapping/charge separation rate and remain constant. The Schatz model, on the other hand, suggests that P680 is a shallow trap which allows exciton feedback to the antenna as centers become closed [Schatz et al., 1987]. Others have also suggested this possibility [Haehnel et al., 1982][Hodges and Moya, 1987][Breton, 1983]. Breton [1983] suggested an alternative explanation which is compatible with the Klimov model by assigning the fast decay component to speedy charge accumulation from $\mathrm{Ph}^{-}$onto $\mathrm{Q}\left(\mathrm{P} 680^{+\cdot} \mathrm{Ph}^{-} \mathrm{Q} \longrightarrow \mathrm{P} 680^{+\cdot} \mathrm{PhQ}^{-}\right)$parallel to the much slower primary charge recombination step. As the population of $\mathrm{Q}^{-}$increases, this charge accumulation step is suppressed, presumably via coulombic effects, leading to a corresponding reduction in the $\tau_{f}$ phase and an increase in emission from both primary charge recombination and, if the temperature is low enough, from $\mathrm{Ph}^{*}$ directly.

Moya reports that $\tau_{\mathbf{s}}$ is 4 -5-fold longer in closed centers [Moya et al., 1986]. Citing these results, Holzwarth points out that it is reasonable to expect a smaller rate constant for primary charge separation considering the presence of the charged species Qin close proximity to $\mathrm{Ph}$ [Holzwarth, 1986]. Both he and Schatz also have correlated 
picosecond absorbance kinetics with the fluorescence decay measurements using low light intensities to specifically minimize any artifactual singlet/singlet annihilation events within the chlorophyll bed [Schatz et al., 1987]. They found that open reaction centers yielded a triexponential absorbance decay while closed centers lacked the longest-lived component, yielding a biexponential decay. This component was attributed to triplet chlorophyll, thus implying low triplet yields in closed centers. They concluded that primary charge separation is curtailed in closed centers. The Klimov model, of course, does not allow for this possibility.

In addition, the slow component has been found in mutant corn chloroplasts with PSII core complexes either missing or greatly depleted [Green et al., 1984]. These researchers suggested that most of the slow phase originates from charge recombination in intact PSII centers, but that it is augmented by direct LHC II emission.

Finally, the activation energy calculated by Klimov for primary charge recombination implies a temperature-dependent rate constant for this process. However, Mathis and Schenck [1982] could not confirm any temperature dependence of $F_{v a r}$; in fact, Mathis [1984] has detected an increasing $F_{v a r}$ at lower temperature.

\section{Energy Distribution Models}

The final fluorescence topic to consider is an explanation for the observed $\mathrm{F}_{\max } / \mathrm{F}_{o}$ intensity ratio. Some typical values reported are 3.0 for $C$. pyrenoidosa and 5.1 for pea chloroplasts [Haehnel et al., 1982]. Values found in this investigation for untreated spinach chloroplasts were approximately 3. One explanation is based upon Butler's [1978] relatively simple "bipartite" model. This model assumes the existence of a general light-harvesting pool consisting of chlorophyll molecules connected to individual PSII reaction centers. It does not distinguish between antennae and LHC, but instead mathematically lumps them together as one light-harvesting antenna unit. According to 
Butler,

$$
\mathrm{F}_{v a r} / \mathrm{F}_{\max } \equiv\left(\mathrm{F}_{\max }-\mathrm{F}_{o}\right) / \mathrm{F}_{\max }=\emptyset_{P \max }
$$

where $\varnothing_{P \max }$ is defined as the maximum photochemistry quantum yield for PSII, observed when all the PSII reaction centers are open. This equation thus relates photochemical yield to fluorescence. Assuming a typical energy loss via fluorescence of $2.2 \%$ and an intersystem crossing (I.C.) loss of $4.5 \%$ to carotenoid pigments [Kramer and Mathis, 1980 ] leads to a reasonable $ø p_{\max }=0.93$. However, substituting this value into the above equation yields a $F_{\max } / F_{o}$ ratio of about 14 , much higher than the 3 to 5 encountered experimentally. Haehnel et al. [1982] has offered some explanations for this discrepancy: (i) A portion of both $\mathrm{F}_{o}$ and $\mathrm{F}_{\max }$ may be so-called "dead" fluorescence originating from chlorophyll which is not coupled to PSII light-harvesting pigments, although his group observed no constant long-lived (2-5 nsec) component in the background [Haehnel et al., 1982]. PSI emission at R.T. is minimal. (ii) The middle decay component, which he attributed to the relatively loosely-coupled LHC II, increased merely by a factor of 1.8 in spinach with closed centers (Table I) and by comparable values in other species [Haehnel et al., 1982]. This nearly constant emission at both $\mathrm{F}_{o}$ and $F_{\max }$ could contribute to the discrepancy. (iii) Haehnel et al. [1982] suggests the possible application of Butler's tripartite model, which postulates an auxiliary radiationless decay pathway [Butler, 1978]. Unlike Butler's bipartite model, this model treats both the LHC II and the antenna chlorophyll as separate entities which interact with each other with specific rate constants. The tripartite model more realistically depicts the core antenna as strongly-coupled to the RC and the LHC II as more loosely-coupled. The concentration of $\mathrm{Mg}^{2+}$ is one factor thought to affect the degree of energy coupling of the LHC II to the RC [Forti, 1987]. According to this view, energy can cycle between the LHC and the antennae or it can travel from the LHC to the RC and thus relegate the antenna to a mediatory role. Although this approach does not encompass Klimov's model directly, 
Haehnel earlier suggested the possibility that primary charge recombination occurs in closed centers if limited to a radiationless process leading to the ground state [Haehnel et al., 1982]. In addition, radiationless decay may occur at three separate locations in the tripartite model as compared to only two for the bipartite model (which ignored distinct antenna types). Despite the sophistication of the tripartite model and its successful application here, however, some believe that the bipartite model is more appropriate for describing fluorescence decay characteristics, especially when combined with the idea of PSII $\alpha$ and $\beta$-center heterogeneity [Geacintov et al., 1986]. 


\section{CHAPTER III \\ MATERIALS AND METHODS}

\section{Fluorescence Study}

Chloroplast Isolation. Chloroplasts were isolated from local market spinach (Spinacia oleracea L.) by grinding spinach leaves in a Waring blender for $60 \mathrm{sec}$ in 400 $\mathrm{mM}$ sucrose, $50 \mathrm{mM}$ TRIS (tris(hydroxymethyl)-aminomethane) and $10 \mathrm{mM} \mathrm{KCl}$ at $\mathrm{pH}$ 7.8 ("STK" buffer solution.). This solution was filtered through Miracloth and then centrifuged for 2 min at $1500 \mathrm{RPM}$ (400g, GSA rotor) to remove gross impurities. The supernatant was then re-centrifuged for $10 \mathrm{~min}$ at $4000 \mathrm{RPM}$ (2500 $\mathrm{g}$, GSA rotor), pelleting the chloroplasts. The pellet was solubilized carefully with STK buffer and the chlorophyll concentration was adjusted using the following procedure according to Arnon [1963]:

1) Approx. $20 \mu \mathrm{l}$ of the chloroplast solution was added to $5 \mathrm{ml} 80 \%$ acetone and then incubated in the dark for $5 \mathrm{~min}$ to extract the chlorophyll.

2) This solution was centrifuged at $5000 \mathrm{RPM}$ for $5 \mathrm{~min}$ to remove insoluble materials.

3) The absorbance of the supernatant was measured on a Hitachi Perkin-Elmer model $139 \mathrm{UV}$-VIS spectrophotometer at $663 \mathrm{~nm}$ and $645 \mathrm{~nm}$, corresponding to absorption peaks for chlorophyll $a$ and $b$, respectively. The chlorophyll concentration was calculated according to the following equation:

$$
\operatorname{chl}(\mu \mathrm{g} / \mathrm{ml})=x\left[(8.02) A_{663}+(20.2) A_{645}\right]
$$

where $\mathrm{x}$ is the dilution factor used. 
$20 \%(\mathrm{v} / \mathrm{v})$ glycerol was added as a cryogenic preservative. The final chlorophyll concentration was $2000 \mu \mathrm{g} / \mathrm{ml}$. The prep was stored in approximately $300 \mu \mathrm{l}$ batches and frozen at $-80^{\circ} \mathrm{C}$. The entire isolation procedure was done under reduced light conditions either in an ice bath or under refrigeration. The chloroplasts were handled gently to minimize damage.

Chemical Reagents. The linolenic acid used in this study was purchased from Sigma Chemical Company and was stored under nitrogen at $-80^{\circ} \mathrm{C}$ as a $35.2 \mathrm{mM}\left(0^{\circ} \mathrm{C}\right)$ ethanolic solution. Ethanolic solutions from 1.4 to $3.2 \mathrm{mM}\left(0^{\circ} \mathrm{C}\right)$ were prepared from the above solution as needed and were stored under identical conditions. 1,5-Diphenylcarbohydrazide (DPC), an artificial electron donor to PSII, was used as a $26 \mathrm{mM}\left(0^{\circ} \mathrm{C}\right)$ ethanolic solution which was stored and used as above. A new solution was prepared whenever any hint of discoloration was detected; the DPC usually recrystallized at $-80^{\circ} \mathrm{C}$, thus retarding deterioration during storage. Final working ethanol concentrations varied from 2 to $9 \%(v / v)(=13$ to $100 \mu \mathrm{M} \mathrm{LA}+$ DPC) for DCIP (2,6-dichlorophenol indophenol) experiments (below) and up to $13 \%$ (= $150 \mu \mathrm{M} \mathrm{LA}+\mathrm{DPC})$ for the remaining experiments. DCMU (3-(3',4'-dichlorophenyl)-1,1-dimethylurea, also known as diuron), an herbicide which inhibits PSII, was pre-prepared as $1 \mathrm{mM}$ methanolic solution and stored at $0^{\circ} \mathrm{C}$. Alcoholic solutions were kept on ice and capped immediately after use to minimize solvent evaporation. Final working concentrations of each reagent were calculated carefully based upon its volume and the volumes of all preceding additions. Working concentrations for chlorophyll, DPC and DCMU were $10 \mu \mathrm{g} / \mathrm{ml}, 500 \mu \mathrm{M}$ and 5 $\mu \mathrm{M}$, respectively. $\mathrm{Mg}^{2+}$ concentrations were $20 \mathrm{mM}$ as $\mathrm{MgCl}_{2}$. Sodium dithionite, $\mathrm{Na}_{2} \mathrm{~S}_{2} \mathrm{O}_{4}$, was added directly from the reagent bottle using a microspatula in sufficient quantity to poise the ambient potential of the sample solution (1-1.5 mg $\Rightarrow 3-4 \mathrm{mM}$ ). Working solutions were made by adding the chloroplast prep and desired additional components to approximately $2 \mathrm{ml}$ buffer solution containing $50 \mathrm{mM}$ HEPES (N-2- 
hydroxylpiperazine-N'-2-ethanesulfonic acid, $\mathrm{pK}_{\mathrm{a}} 7.5$ ) and $0.33 \mathrm{M}$ sorbitol in a $1 \mathrm{~cm}$-path $3 \mathrm{ml}$ cuvette. The cuvette was gently agitated prior to each trial in order to counteract the effects of chloroplast settling. Final $\mathrm{pH}$ was checked carefully on an Orion model SA 720 $\mathrm{pH}$ meter to ensure its consistency after component additions. Measurements were made using a Sigma "TRIZMA" pH electrode because of its precision and accuracy in systems utilizing biological buffers and because its small physical diameter enabled $\mathrm{pH}$ measurements of small sample volumes within cuvettes. $\mathrm{pH}$ adjustments were made with $\mathrm{HCl}$ and $\mathrm{NaOH}$.

Apparatus. Chlorophyll fluorescence was measured at room temperature on a labconstructed spectrofluorometer (Fig. 2, below). The apparatus consisted of a continuous actinic light source (150 watt tungsten-iodide General Electric \#1958 bulb in a fan-cooled Oriel lamp housing), a grating monochromator (Minimate model 1650), and a redsensitive photomultiplier tube detector operated at room temperature. The monochromator was adjusted to approximately $490 \mathrm{~nm}$ and then fine-tuned for maximum fluorescence signal output. Slits were used at both the entrance and exit of the monochromator; these were chosen in conjunction with various lenses such that beam geometry at the $1 \mathrm{~cm}$-path cuvette was optimized. A blue filter (lambda $\max =488 \mathrm{~nm}$ ) was used between the monochromator and sample cuvette in order to prevent red spectral overtones from interfering with fluorescence emission detection. An electro-mechanical shutter ( $3 \mathrm{msec}$ opening time) was placed in the beam between this filter and the cuvette. The excitation beam intensity was insufficient to saturate the sample. A red $657 \mathrm{~nm}$ cut-off filter was placed between the sample and detector to prevent any stray light from the excitation beam from reaching the detector. Detector cathode voltage was supplied by a Hewlett-Packard model $6515 \mathrm{~A}$ dc power supply and was maintained at a constant $-700 \mathrm{~V}$, as monitored on a Keithley model 163 digital voltmeter. Detector output was amplified using a preamp (EG\&G/PARC model 113: dc-coupled, $\mathrm{HF}$ roll-off $=30 \mathrm{~Hz}, 10 \mathrm{x}$ gain) and then fed into 
a single-board process control computer ("Elf II" by Netronics Research and Development, Ltd.). The computer was programmed in FORTH to trigger the digital transient recorder $110 \mathrm{msec}$ prior to shutter opening (as monitored on an Anadex model CF-300R digital timer) in order to establish a baseline. Data were collected on a Biomation model 802 digital transient recorder utilizing a dual switched time-base format to collect 1024 data points per trial. Signal strength $v s$. time was viewed concurrently on a Tektronix model 620 oscilloscope. The dual-time format was selected in order to reveal detail immediately prior to and following opening of the shutter while retaining the ability to record and observe the overall fluorescence event, which lasted more than 2 seconds.

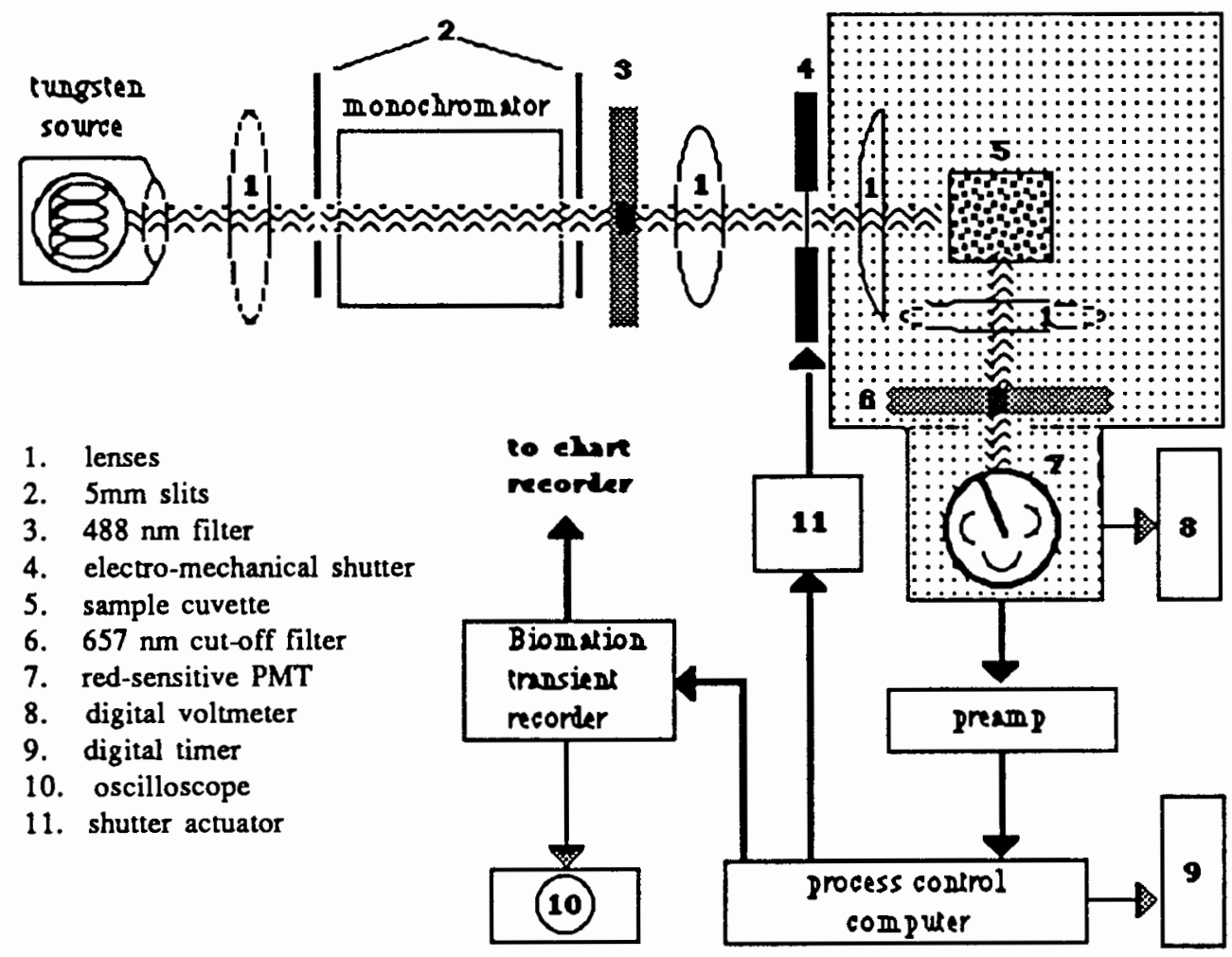

Figure 2. Spectrofluorometer apparatus.

Sweep time bases"A" and "B" were set at $1 \mathrm{sec}$ and $5 \mathrm{sec}$, respectively, with a delay setting of 1.7 , corresponding roughly to 200 total points for $200 \mathrm{msec}$ at 1024 points/sec for "A," and 820 total points for $4 \mathrm{sec}$ at 200 points/sec for "B." Other Biomation settings 
were $200 \mathrm{mVdc}$ full-scale input and external neg. slope ac-trigger. Stored data were plotted directly from the Biomation after each trial on a Hewlett-Packard model 7004B XY recorder set at $50 \mathrm{mV} / \mathrm{cm}$ and $10 \mathrm{sec} / \mathrm{in}$.

\section{Flash Photolysis}

Photosystem II Particles. The photosynthesis prep used in this portion of the investigation was prepared from wheat chloroplasts and frozen at $-80^{\circ} \mathrm{C}$ by David Becker ${ }^{2}$ and consisted of $\mathrm{O}_{2}$-evolving everted (inside-out) thylakoid membrane stacks containing predominantly PSII. The prep was added directly to $3.0 \mathrm{ml}$ of $\mathrm{pH}$-adjusted buffer solution in a $3 \mathrm{ml}$ cuvette. Final chlorophyll concentration was between 10 and $15 \mu \mathrm{g} / \mathrm{ml}$; the exact chlorophyll concentration was unimportant since this study measured the rate of change of $\mathrm{P} 680^{+}$concentration.

Chemical Reagents. Both the linolenic acid and $\mathrm{MgCl}_{2}$ solutions were the same ones used in the fluorescence study (above). Buffer solutions for $\mathrm{pH} 7.6$ and 6.8 were the same as for the fluorescence study. Potassium dihydrogen phosphate buffer $\left(\mathrm{pK}_{\mathrm{a}} 6.8\right)$ was used for $\mathrm{pH} 6.0$ trials $\left(0.33 \mathrm{M}\right.$ sorbitol $\left.+50 \mathrm{mM} \mathrm{KH}_{2} \mathrm{PO}_{4}\right)$. The sample cuvette was agitated vigorously prior to measurement in order to distribute the aggregate-prone prep homogeneously. Sodium tetraphenylborate (TPB, a fast artificial donor to PSII) was added from a fresh $1 \mathrm{mM}$ stock aqueous solution to a final working concentration of 10 $\mu \mathrm{M}$, in order to elucidate any flash artifact for subsequent subtraction. Ferricyanide was added as its potassium salt from a freshly made $1 \mathrm{M}$ stock aqueous solution to a final concentration of $1 \mathrm{mM}$ in order to elucidate any residual PSI contribution to the absorption transient.

Apparatus. Flash-induced absorption transients were performed at $820 \mathrm{~nm}$ $\left(\mathrm{P} 680^{+}\right)$on a lab-constructed spectrophotometer (Fig. 3). The reference source consisted

2 Currently at Pomona College in Claremont, CA. 
of a 150 watt tungsten-iodide General Electric \#1958 bulb in a 66000 series Oriel lamp housing. The reference beam was passed through an entrance grating monochromator (Jarrell-Ash, model 82-410) and then through a $760 \mathrm{~nm}$ cut-off interference filter before reaching the cuvette. After passing through the $1 \mathrm{~cm}$-path cuvette, the reference beam was passed through an exit $760 \mathrm{~nm}$ cut-off filter to prevent any laser flash from reaching the exit monochromator and detector, and then an exit monochromator (Jarrell-Ash, model 82410) before reaching the measuring detector (Pin-10D Schottky barrier photodiode by United Detector Technology). Both monochromators were calibrated using an $820 \mathrm{~nm}$ interference filter. Saturating flashes were performed with a Phase-R model DL-1200 v flashlamp-pumped dye laser utilizing sulforhodamine-101 dye in methanol to produce a $150 \mathrm{~mJ}, 400 \mathrm{nsec}(\mathrm{FWHM})$ pulse at $650 \mathrm{~nm}$. Pulses were spaced approximately $3 \mathrm{sec}$ apart. The laser was isolated in a separate room from the spectrophotometer to minimize interference with the data collection electronics. The beam was directed to the cuvette using a mounted prism. Output from the detector was passed through two EG\&G/PARC model 113 preamps: preamp " $\mathrm{A}$ " was dc-coupled with gain $=10$, low frequency roll-off set at dc and high frequency roll-off set at $300 \mathrm{kHz}$. Baseline signals, designated $\mathrm{I}_{1}$ values, were measured with the sample in place and then recorded prior to each laser flash sequence from the output of this amplifier using a Fluke model $8200 \mathrm{~A}$ digital voltmeter. Preamp "B" was ac-coupled with low and high frequency roll-off settings of $0.3 \mathrm{~Hz}$ and $300 \mathrm{kHz}$, respectively, with gain adjustable from $1 \mathrm{~K}$ to $20 \mathrm{~K}$ as convenient. The time response of the spectrometer was limited to $\approx 4 \mu \mathrm{s}$ by the $300 \mathrm{kHz}$ bandwidth of the preamps. Output from B was fed into a Nicolet model 4094A Digital Oscilloscope (dccoupled, 16000 data points at $0.5 \mu$ s per point, $\pm 100 \mathrm{mV}$ for a total full-scale setting of $200 \mathrm{mV}$ ). The oscilloscope was triggered by a portion of the laser beam reflected off the cuvette face and onto a photodiode. The laser flash sequence was triggered by a SYM-1 single-board microcomputer programmed in FORTH and modified in-house. The 
computer was used in conjunction with a Tektronix 4112 terminal.

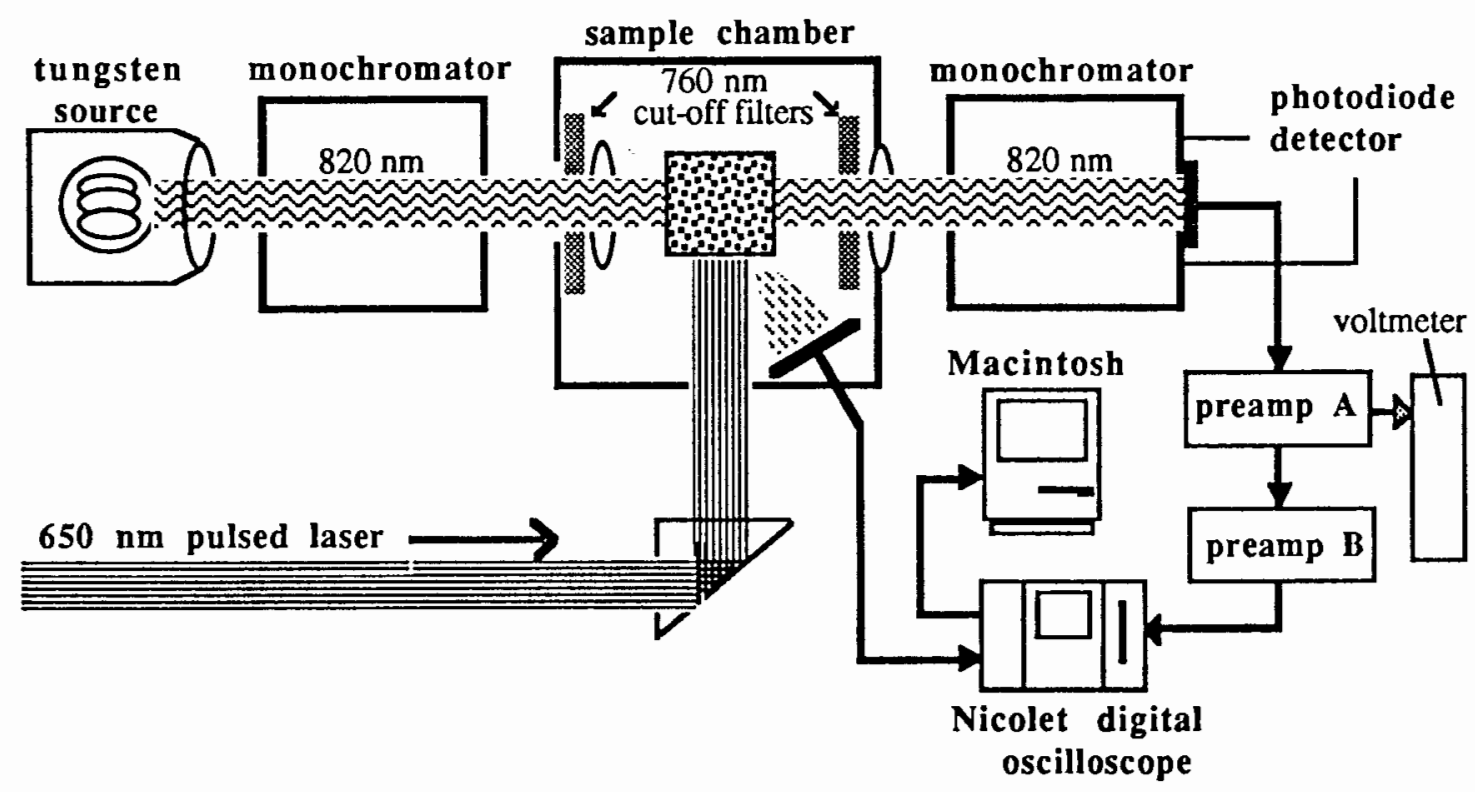

Figure 3. P- $680^{+}$flash photolysis spectrophotometer.

Data Aquisition. The data for each 16 flash sequence were averaged on the Nicolet during acquisition, stored, and then transferred to a Macintosh 512E computer for further manipulation and storage. ${ }^{3}$ While 16000 data points were stored on the Nicolet per plot, only 2000 points per plot were transferred to the Macintosh, translating into a net effective data acquisition speed of $2 \mu \mathrm{s}$ per point. This was done in order to conserve disk storage space and to increase processing speed; it led to no discernible loss of resolution. Each absorption plot on the Macintosh was normalized to $50 \mathrm{mV}$ dc prior to storage by multiplying the data by a constant value equal to $50 / \mathrm{I}_{1}, \mathrm{I}_{1}$ having been measured previously for each individual trial (above). The value $50 \mathrm{mV}$ was chosen both because of its proximity to the original $I_{1}$ response values and because it is large enough to allow for

3 The programs used for data acquisition and manipulation on the Macintosh were designed and written in FORTH by Martin Corera, an undergraduate Chemistry student at PSU. 
simplification of the transient absorbance change calculation. Calculations were based on a [P680+] absorptivity of $6500 \mathrm{M}^{-1} \mathrm{~cm}^{-1}$ at $820 \mathrm{~nm}$.

\section{DCIP Bleaching Kinetics}

Chloroplast Prep. The chloroplast prep used for this study was the same one used for the fluorescence study (above).

Chemical Reagents. Buffers and reagents used in this study, with the exception of DCIP (2,6-dichloroindophenol), were the same ones used in the fluorescence study unless otherwise stated. DCIP was added as its sodium salt directly to buffer solution (50 mM HEPES + $330 \mathrm{mM}$ sorbitol) which was then filtered using Whatman \#1 filter paper to remove any undissolved impurities. The $\mathrm{pH}$ was then adjusted using $\mathrm{NaOH} / \mathrm{HCl}$ (Orion model SA 720 equipped with a "TRIZMA" pH-electrode). As a result of a natural bleaching process observed in stored dye solutions, only dye solutions less than 48 hours old were used. pH 6.0 solutions were used within 8 hours because they tended to bleach very rapidly while in storage. This is consistent with observations by Clark [1960] who reported that DCIP decomposes in acidic solutions. Solutions were kept at $4^{\circ} \mathrm{C}$ until ready to use in order to retard bacterial growth, at which point they were warmed to room temperature in a microwave oven. All bleaching experiments were done at room temperature. DCIP absorptivities are $\mathrm{pH}$-dependent and needed to be determined at 568 $\mathrm{nm}$ for $\mathrm{pH} \mathrm{6,6.8} \mathrm{and} \mathrm{7.6,} \mathrm{the} \mathrm{values} \mathrm{used} \mathrm{in} \mathrm{the} \mathrm{experiment.} \mathrm{The} \mathrm{combination} \mathrm{of} \mathrm{an}$ extremely high absorptivity for DCIP and relatively impure crystals made an indirect determination most feasible. First, four buffered DCIP solutions taken from a freshly made parent solution were carefully adjusted to the above $\mathrm{pH}$ values and to $\mathrm{pH} 6.5$ and their absorbances were measured in a Cary-14 Spectrophotometer at $568 \mathrm{~nm}$ and $600 \mathrm{~nm}$. According to Flexser et al. [1935], 


$$
\varepsilon_{600, \mathrm{pH}}=\frac{\left(\frac{\mathrm{K}^{\prime \prime}}{\mathrm{aH}^{\prime}}\right) \varepsilon_{\mathrm{A}}+\varepsilon_{\mathrm{HA}}}{1+\left(\frac{\mathrm{K}^{\prime}}{\mathrm{aH}_{+}}\right)}
$$

where $\varepsilon_{600, \mathrm{pH}}$ is the observed DCIP absorptivity at $600 \mathrm{~nm}, \varepsilon_{\mathrm{HA}}$ and $\varepsilon_{\mathrm{A} \text { - }}$ are the absorptivities for the protonated and unprotonated forms, respectively, $\mathrm{K}^{\prime}$ is the apparent dissociation constant and aH+ is the hydrogen ion activity at the desired $\mathrm{pH}$. According to Armstrong [1964], $\varepsilon_{\mathrm{HA}}=2.7 \pm 0.1, \varepsilon_{\mathrm{A}^{-}}=22.0 \pm 0.1$ and $\mathrm{pK}^{\prime \prime}=5.90 \pm 0.02$ for $\mathrm{pH}$ $5.2-6.7$ and $600 \mathrm{~nm}$. Substitution of these values into the above equation yields $\varepsilon_{600,6.5}$ $=18,100 \mathrm{M}^{-1} \mathrm{~cm}^{-1}$. Multiplying this value by $\boldsymbol{H}_{568, \mathrm{pH}} / \boldsymbol{H}_{600,6.5}$, determined from direct measurements of the $\mathrm{pH}$-adjusted solutions, yields $\varepsilon_{568,6.0}=12,600, \varepsilon_{568,6.8}=16,400$ and $\varepsilon_{568,7.6}=18,000 \mathrm{M}^{-1} \mathrm{~cm}^{-1}$. Accurate absorptivities were necessary in order to determine acceptably accurate DCIP bleaching rates. Consistent initial dye concentrations were considered less essential, with DCIP concentrations ranging from 36-44 $\mu \mathrm{M}$, confirmed spectrometrically.

The chloroplast prep was added directly to the dye solutions which were then gently agitated immediately prior to measurement.

Apparatus. Bleaching experiments were performed on a lab-constructed spectrophotometer utilizing a Beckman DU (model 2400) monochromator equipped with a tungsten lamp excitation source (figure 4). 


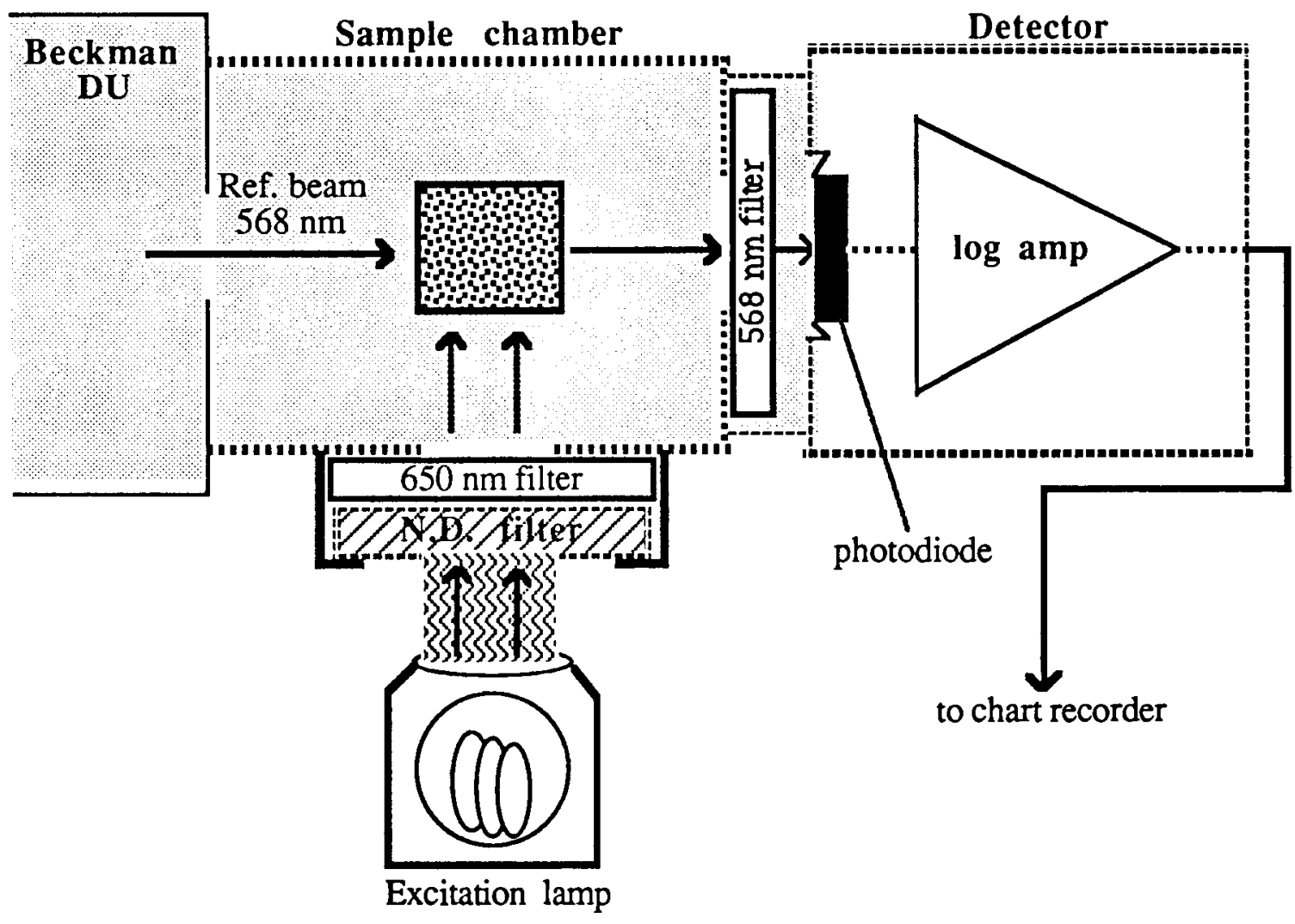

Figure 4. Spectrophotometer to monitor DCIP bleaching kinetics.

Slit width was adjusted to $0.2 \mathrm{~mm}$. Sample size was maintained at approximately $1 \mathrm{ml}$ in a $3 \mathrm{ml} 1 \mathrm{~cm}$-path cuvette, presenting a comparable sample cross section to both excitation and reference beams; this was done to minimize diffusional effects encountered when the beam : sample area ratio was less than unity. Sample excitation was accomplished with a Unitron "LKR" adjustable microscope source housing a 40 watt tungsten lamp. The excitation beam was passed through a $650 \mathrm{~nm}$ wide-band interference filter before striking the cuvette at $90^{\circ}$ to the measuring beam . Excitation beam attenuation was accomplished by placing a desired neutral density filter prior to and adjacent to the $650 \mathrm{~nm}$ filter. The three available neutral density filters had absorbances of $0.26(55 \% \mathrm{~T}), 0.47(34 \% \mathrm{~T})$ and $0.91(12 \% \mathrm{~T})$, as measured on a Cary-14 spectrophotometer at $650 \mathrm{~nm}$. A $568 \mathrm{~nm}$ interference filter was placed in the measuring 
beam after the sample cuvette, preventing any scattered excitation light from reaching the detector. The monochromator wavelength was adjusted for maximum detector output with sample removed.

The detector used was an RCA CA-3021 photodiode operated in a short-circuit current mode with output fed directly into to a solid state log ratio amplifier (Analog Devices model 757P) (See Fig. 5). ${ }^{4}$ As the Beckman DU is a single beam instrument, whereas the log amp module was designed for dual beam applications with both signal and reference inputs, a reference current source was constructed by attaching to the reference channel a GaAsP light-emitting diode in series with a $1 \mathrm{M} \Omega$ resistor. Because the reference input channel was designed with nearly zero input impedance, the stable $2.01 \mathrm{~V}$ drop across the LED produced a constant $2 \mu \mathrm{A}$ reference current. The log amp was configured for an output of -1 volt per decade change input current. Additional flexibility was provided by the addition of an operational amplifier (op amp \#741) in an adjustable gain configuration. An externally mounted offset potentiometer was connected to a zenerregulated voltage divider circuit, allowing easy selection of either negative or positive output offset voltages. Gain was adjusted with an externally mounted $100 \mathrm{~K} \Omega$ feedback potentiometer, allowing a range of approximately 1.2 to 11.2 , thus leading to a net overall log scale factor of -1.2 to -11.2 volts per decade. Maximum gain was typically employed during experimentation. Detector output was plotted vs. time on an Omniscribe chart recorder (Houston Instruments). Detector linearity was verified by a plot of output vs. concentration for DCIP solutions previously measured on a Cary-14 spectrophotometer at $568 \mathrm{~nm}$. Offset adjustments were determined to have minimal impact on either gain or linearity. The entire apparatus, including chart recorder, was insulated from supply line fluctuations using a constant voltage transformer (Sola Electric).

4 The detector circuit was designed and assembled by Chuck Haymond (while an undergraduate Chemistry student at PSU) and myself. 


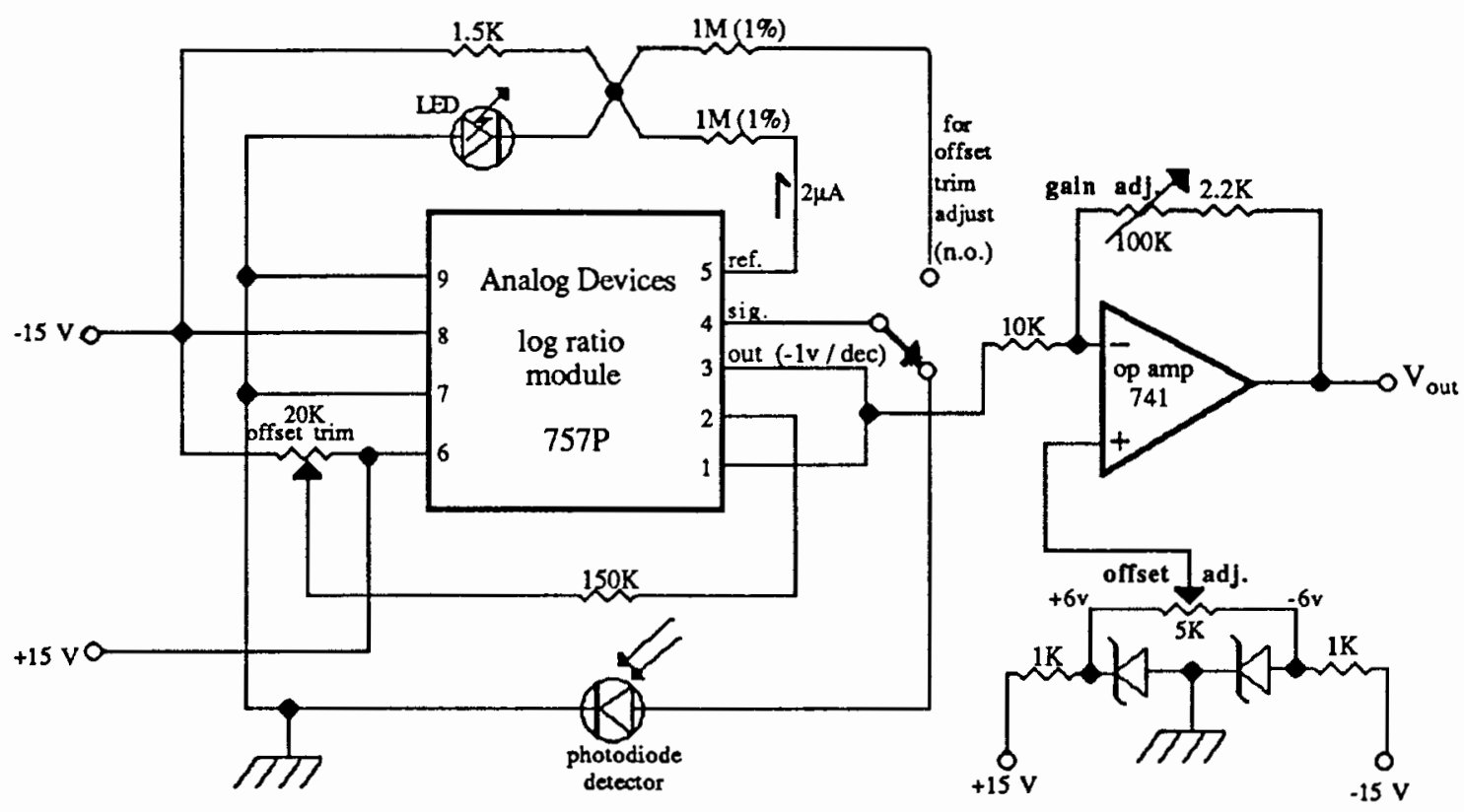

Figure 5. Detector circuitry for DCIP bleaching spectrophotometer.

Bleaching rates were determined by direct measurement of the slopes of chart recorder bleaching plots. Calibration of system response was accomplished using the same DCIP solution used for the experiment, but without any chloroplast addition. An absorbance value for the solution was first determined on a Cary-14 spectrophotometer with the reference beam unperturbed. The response of the experimental spectrophotometer was then determined for the same calibration sample with respect to an unperturbed monitoring beam. Since the normal $1 \mathrm{~V}$ full-scale recorder setting was too sensitive for such a large absorbance difference, calibration was done in the $10 \mathrm{~V}$ range setting. The absorbance necessary to give full-scale pen deflection was then easily calculated and this value was divided by 10 to determine the full-scale value for the more sensitive range setting used in the experiment. Recorder range settings were verified previously for accuracy using a Power Designs model 5005R dc-power supply. Although experience confirmed excellent detector circuit stability over time, calibration measurements were 
repeated every $30-60 \mathrm{~min}$ in the manner described above. Chart speeds were chosen for convenience, i.e., faster bleaching rates required faster chart speeds in order to produce slopes shallow enough to reduce subsequent measuring errors. 


\section{CHAPTER IV}

\section{DATA PRESENTATION}

\section{Fluorescence}

Fluorescence measurements constitute a useful tool for monitoring the emitting states of pigments associated with the PSII reaction center. Perturbation of intact systems for the purpose of studying the effects upon fluorescence include varying the excitation light intensity and/or wavelength, changing the ambient redox potential, and affecting the influence of selected portions of PSI and PSII either by using various types of chemical inhibitors or activators, by alteration via genetic engineering methods, or by direct removal using detergents and/or mechanical means.

Single photon timing (SPT) on a time scale of picoseconds or less is a relatively new technique which offers unprecedented opportunities to elucidate the multiple energy trapping steps in PSII. A summary of the current understanding of results generated by this method for plants and algae was presented in Chapter II. Alternatively, steady-state fluorescence measurement is a much simpler technique than SPT and has thus often been used to monitor the state of the PSII reaction center. The current fluorescence study utilizes steady-state measurements alone.

Relatively high, saturating light intensities are usually used to study fluorescence since this allows area measurements above induction rise curves to be used as a tool for interpreting fluorescence data. At saturating light intensities the PSII trapping rate remains at a constant level and the area above the induction rise curve area is inversely proportional to the amount of accumulated $\mathrm{Q}_{\mathrm{A}}{ }^{-}$present - assuming no additional quenchers exist. The steady-state $Q_{A}{ }^{-}$level depends in turn upon the rate of donation to the cyt $b_{6} / f$ complex by 
the pool of plastoquinone (PQ) acceptors following $\mathrm{Q}_{\mathrm{B}}$ in the electron transport chain. This electron donation from $\mathrm{PQH}_{2}$ to cyt $\mathrm{b}_{6}$ is the rate-limiting step in PSII. The above approach is not applicable here, however, since the light intensity used in this work was non-saturating. Consequently, fluorescence results are discussed only in terms of $F_{i}$ (or $\mathrm{F}_{o}$ ), $\mathrm{F}_{\max }$, and their ratio. $\mathrm{F}_{o}$ refers to the initial fluorescence level from control samples, whereas $F_{i}$ refers to the initial level in non-control samples and is typically greater than $F_{o}$ (Fig. 6).

Rise curves for untreated controls are time-stable, with a constant $\mathrm{F}_{o}$ and $\mathrm{F}_{\max }$ level. Although Renger observed an $\mathrm{F}_{\max }$ decline in oxygen-evolving spinach PSII particles, which he attributed to the photoaccumulation of $\mathrm{Ph}$, this required pre-treatment with dithionite and relatively intense actinic illumination [Renger et al., 1983].

At $\mathrm{pH} 7.6,150 \mu \mathrm{M}$ LA causes an immediate $\mathrm{F}_{i}$ rise to the same level as $\mathrm{F}_{\max }$, whereas $67 \mu \mathrm{M}$ LA achieves this only after a 5 minute incubation (Figs. 6-9). As in DCMU-treated samples at $\mathrm{pH} 7.6,150 \mu \mathrm{M}$ LA fails to raise $\mathrm{F}_{i}$ to the $\mathrm{F}_{\max }$ level at $\mathrm{pH} 6$ under normal conditions (below) (Fig. 10). The abbreviated LA-induced $F_{i}$ rise supports the observation by Vernotte and co-workers that LA and DCMU exhibit similar effects upon fluorescence induction at $\mathrm{pH}$ 6. However, whereas the Vernotte group found no LA-induced $\mathrm{F}_{i}$ rise at $\mathrm{pH} 6$ [Vernotte et al., 1983], this study found $\mathrm{F}_{i}$ raised to $60 \%$ of $\mathrm{F}_{\max }$ after 5 minute incubation with $150 \mu \mathrm{M} \mathrm{LA}$ at $\mathrm{pH} 6$ (Fig. 10), and to $36 \%$ of $\mathrm{F}_{\max }$ after 5 minutes with DCMU (Fig. 11).

As shown in Figs. 8 and 12, the $F_{i} v s$. [LA] curve is bimodal after a 5 minute incubation period, with a small peak in $F_{i}$ around $33 \mu \mathrm{MLA}$, followed by a dip centered at approx $50 \mu \mathrm{M}$, followed in turn by a major rise at higher [LA]. Samples incubated with LA for only $20 \mathrm{sec}$, as well as those co-incubated with $\mathrm{Mg}^{2+}$, lack the small $\mathrm{F}_{i}$ peak (cf. Figs. $8,12,13 a, b)$. In general, co-incubation with $\mathrm{Mg}^{2+}$ leads to an $\mathrm{F}_{i}$ response that is more time-stable, which can be seen by comparing the $20 \mathrm{sec}$ and 5 minute curves in Figs. 
13a,b with the comparable curves in Figs. 8, 12. This time stability is also reflected in the $F_{\max }$ level. The relative constancy of both $\mathrm{F}_{\max }$ and $\mathrm{F}_{i}$ with $\mathrm{Mg}^{2+}$ in the lower [LA] region $(<33 \mu \mathrm{M})$ leads to a much more stable $\mathrm{F}_{\text {max }} / \mathrm{F}_{i}$ ratio in this region as well. $\mathrm{Mg}^{2+}$ also causes higher $\mathrm{F}_{i}$ levels near $100 \mu \mathrm{M} \mathrm{LA}$, but these high levels decay significantly to near the non- $\mathrm{Mg}^{2+}$-treated sample levels after a 5 minute incubation with $150 \mu \mathrm{M} \mathrm{LA}$, probably due to grana destacking (see Discussion). The $F_{\max }$ level drops just as quickly as $F_{i}$ since $F_{i}=F_{\max }$ at these $L A$ concentrations, but the plots do not reflect this since $F_{i}$ and $F_{\max }$ have been normalized separately to their control counterparts (all plots of the $\mathrm{F}_{\text {max }} / \mathrm{F}_{i}$ ratio are actual, unnormalized values unless otherwise indicated). A $20 \mathrm{sec}$ incubation period in the presence of $\mathrm{Mg}^{2+}$ is apparently not sufficient to produce a similar rapid decline in F intensities, either with or without DPC (Figs. 13a,b). The time profile for $150 \mu \mathrm{M} \mathrm{LA}$ (Fig. 14) reveals more clearly that the initial $\mathrm{F}_{i}$ and $\mathrm{F}_{\max }$ levels with $\mathrm{Mg}^{2+}$ are above those for samples without $\mathrm{Mg}^{2+}$ but that these drop subsequently to non- $\mathrm{Mg}^{2+}$ treated levels in approximately 15 minutes when $(150 \mu \mathrm{M}) \mathrm{LA}$ is present, with the fastest decline occurring within the first 5 minutes.

The observed effects upon fluorescence of various reagents, alone and in combination with one another and with LA, follows:

DPC $(500 \mu \mathrm{M})$. This agent is an artificial electron donor to PSII. Since it either enhances the oxygen-evolving complex (OEC) or bypasses it all together, it can give an indication of the extent of influence of the OEC upon fluorescence emission. Results indicate that DPC exhibits a minimal overall effect on fluorescence. It also shows a minimal effect upon $\mathrm{P} 60^{+}$absorption transients (see below). This agent primarily lowers $\mathrm{F}_{\max }$ in both control and LA-treated samples, with little effect upon $\mathrm{F}_{i}$. Consequently, $\mathrm{F}_{\text {max }} / \mathrm{F}_{i}$ levels normalized to the control are nearly the same $\pm \mathrm{DPC}$, whereas unnormalized values are initially lower by $10-15 \%$ in DPC-treated samples (Figs. 8,12). This effect is not as apparent in samples with $\mathrm{Mg}^{2+}$ (Figs. 13a,b). Surprisingly, $\mathrm{F}_{\text {var }}$ was diminished 
greatly (i.e., $F_{i} \approx F_{\max }$ ) in the presence of $150 \mu \mathrm{M}$ LA at pH 6 only when DPC was present (data not shown).

Dithionite, $\mathrm{S}_{2} \mathrm{O}_{4}{ }^{2-}(3-4 \mathrm{mM})$. This agent is a strong reductant $\left(E_{\mathrm{m}}=-420 \mathrm{mV}\right.$ at $\mathrm{pH} 7$ ) and is used here to study the effects of chemical reduction of PSII. Reduction is known to extend to the secondary quinone acceptor $Q_{A}\left(E_{m}=-130 \mathrm{mV}\right.$ ?). Dithionite raises $\mathrm{F}_{\max }$ immediately by $46 \%$ in the presence of $\mathrm{Mg}^{2+}$ and $55 \%$ without $\mathrm{Mg}^{2+}$, where it remains relatively constant (Figs. 15a,b). Both the intensity of this emission and its constant character indicate that this is not due to addition luminescence afterglow [ $c f$. Velthuys and Amesz, 1973]. The effect upon $F_{i}$ is time-dependent: $20 \mathrm{sec}$ after addition, the dithionite rise curve appears similar in appearance to the DCMU case (below), but with a slightly higher $F_{i}$ and $F_{m a x}$. However, unlike DCMU, dithionite causes $F_{i}$ to continue rising by $\approx 360 \%$ in the presence of $\mathrm{Mg}^{2+}$ and by $\approx 300 \%$ without $\mathrm{Mg}^{2+}$ after 15 minutes where it too remains relatively constant, $\pm 10 \%$ (Figs. $15 \mathrm{a}, \mathrm{b}$ ). The small sigmoidal plateau observed with control samples after reaching $\mathrm{F}_{o}$ largely and immediately disappears with dithionite, and the unnormalized $F_{m a x} / F_{i}$ ratio is reduced to 1.1 (i.e., to near unity) after 15 minutes, indicating that the small remaining variable phase approaches a value close to zero as time progresses. Thus, dithionite-induced fluorescence appears LA-like, except that a minor variable phase remains with dithionite, the process takes longer, and the fluorescence intensity is higher (Figs. 15a,b). Velthuys reported a dithionite-induced $\mathrm{F}_{i}$ rise by only $60 \%$, to $160 \%$ of control's $\mathrm{F}_{o}$, which is only about $30 \%$ of the rise observed in the current work [Velthuys \& Amesz, 1974].

In the presence of dithionite alone, $F_{\max }$ has been reported [Renger et al., 1983] to drop quickly - by $70 \%$ in $20 \mathrm{sec}$ - during high illumination, attributed to $\mathrm{Ph}^{-}$ photoaccumulation in the presence of chemically reduced $Q_{A}$. This effect was not observed in this study, however, presumably due to the low light intensity employed (Figs. 15a,b). 
Addition of dithionite to samples pre-treated for 5 minutes with $150 \mu \mathrm{M}$ LA $\left(+\mathrm{Mg}^{2+}, \mathrm{pH} 7.6\right)$ maintains the $\mathrm{LA}$-induced lack of a visible $\mathrm{F}_{\text {var }}\left(\right.$ i.e., $\left.\mathrm{F}_{i}=\mathrm{F}_{\max }\right)$, but causes an immediate rise in fluorescence intensity by a constant absolute value of $31 \%$ in the presence of $\mathrm{Mg}^{2+}$ and of $22 \%$ without $\mathrm{Mg}^{2+}$, with normalized values for the $+\mathrm{Mg}^{2+}$ case $\approx 83 \%$ of control's $F_{o}$ and $\approx 27 \%$ of control's $F_{\max }$ (Fig. 16). In addition, the linear rate of fluorescence decline following addition is the same in both cases (data for $+\mathrm{Mg}^{2+}$ only).

Addition of dithionite to samples pre-treated initially with both $150 \mu \mathrm{M} \mathrm{LA}$ and 5 $\mu \mathrm{M}$ DCMU (data not shown) lacked a visible $\mathrm{F}_{v a r}$ and raised fluorescence intensity by $\approx 46 \%$ in the presence of $\mathrm{Mg}^{2+}$ and $27 \%$ without $\mathrm{Mg}^{2+}$. This was equivalent to $66 \%$ and $45 \%$ of the control's $\mathrm{F}_{o}$ (with and without $\mathrm{Mg}^{2+}$, respectively) and to $20 \%$ and $14 \%$ of the control's $\mathrm{F}_{\max }$ (with and without $\mathrm{Mg}^{2+}$, respectively).

Addition of $150 \mu \mathrm{M}$ LA to samples pre-treated with dithionite (Figs. 15a,b) produces the immediate loss of visible $\mathrm{F}_{\text {var }}$ seen normally with LA treatment. Total fluorescence intensity is lowered immediately in the absence of $\mathrm{Mg}^{2+}$ but more slowly in the presence of $\mathrm{Mg}^{2+}$. This decline in intensity with $\mathrm{Mg}^{2+}$ has a similar profile as that observed when LA was the sole agent (cf. Figs. $14 \& 15 \mathrm{a}$ ), but is $70 \%$ faster in the linear region. The initial decline reflects about the same relative intensity drop as the immediate decline seen initially without $\mathrm{Mg}^{2+}$ (Figs. 15a,b).

Addition of dithionite to samples pre-treated with $5 \mu \mathrm{M}$ DCMU alone (data not shown) caused an immediate absolute $\mathrm{F}_{\max }$ rise of $18 \%\left( \pm \mathrm{Mg}^{2+}\right)$ and an $\mathrm{F}_{o}$ rise rate slowed by $\approx 50 \%$ compared to dithionite alone $\left( \pm \mathrm{Mg}^{2+}\right)$; therefore, dithionite appears able to mask the effects of DCMU.

DCMU $(5 \mu \mathrm{M})$. This agent is a potent herbicide which is known to inhibit PSI by displacing $\mathrm{Q}_{\mathrm{B}}$ from its binding site on D1 (Fig. 1). Its site specificity allows better identification of the effects and activity sites of other agents and perturbations within PSII. 
Results indicate that DCMU confers the most time-stable fluorescence effects of any substance tried. Within $20 \mathrm{sec}$ it raises $\mathrm{F}_{\max }$ by $\approx 20 \%$ in the presence of $\mathrm{Mg}^{2+}$ and by $36 \%$ without $\mathrm{Mg}^{2+}$, and $\mathrm{F}_{i}$ by $\approx 23 \%\left( \pm \mathrm{Mg}^{2+}\right.$ ) (Figs. $17 \mathrm{a}, \mathrm{b}$ ). The $\mathrm{F}_{\max }$ level remains essentially constant and the $\mathrm{F}_{o}$ level rises by only $\approx 1.5 \% / \mathrm{hr}$. The sigmoidal plateau observed in control samples at $\mathrm{F}_{o}$ largely disappears upon DCMU addition, but an induction phase remains. Doubling the concentration of DCMU to $10 \mu \mathrm{M}$ showed identical results (data not shown).

Unlike the case with dithionite, addition of DCMU to samples pre-treated with 150 $\mu \mathrm{M} \mathrm{LA}\left(+\mathrm{Mg}^{2+}\right)$ does not cause a rise in fluorescence intensity and may reduce the rate of decline slightly (Fig. 18). Addition of DCMU to samples pre-treated with dithionite has little effect upon $F_{\max }$ but raises $F_{i}$ by the same fraction as in samples treated with DCMU alone, i.e., by $50 \%$ of control's $\mathrm{F}_{o}$ after 10 minutes (data not shown). Addition of 150 $\mu \mathrm{M}$ LA to samples pre-treated with DCMU produces both the loss of visible $\mathrm{F}_{\text {var }}$ and subsequent decline rate in fluorescence intensity seen typically in samples treated only with $\mathrm{LA}\left( \pm \mathrm{Mg}^{2+}\right)($ data not shown $)$.

The smaller $\mathrm{F}_{i}$ intensity induced by LA at lower $\mathrm{pH}$ (above) leads to a rise curve similar in appearance to DCMU-mediated curves (cf. Figs. $10 \& 11$ ). This fact, along with LA induced DCMU-like afterglow luminescence, led Vernotte to conclude that LA exhibits DCMU-like inhibition between $\mathrm{Ph}$ and $\mathrm{Q}_{\mathrm{A}}$, causing an analogous shift in acceptor-state equilibrium [Vernotte et al., 1983].

The following is a summary of the effect upon fluorescence of the various agents discussed above for $\mathrm{pH} 7.6$ and $150 \mu \mathrm{M} \mathrm{LA}$ :

1.) LA: At $\mathrm{pH} 7.6,150 \mu \mathrm{M}$ LA raises $\mathrm{F}_{i}$ immediately to $\mathrm{F}_{\max }\left( \pm \mathrm{Mg}^{2+}\right)$. This effect is much less pronounced at $\mathrm{pH} 6$.

2.) $\mathrm{S}_{2} \mathrm{O}_{4}{ }^{2-}: \mathrm{F}_{\max }$ is raised immediately by $\approx 50 \% ; \mathrm{F}_{i}$ is raised more slowly by $\approx 360 \%$ and $300 \%$ with and without $\mathrm{Mg}^{2+}$, respectively, after 15 minutes. 
3.) $\mathrm{S}_{2} \mathrm{O}_{4}{ }^{2-}+\underline{\mathrm{LA}}$ : LA vitiates the observable dithionite fluorescence effects entirely. The curve appears like a typical LA curve with $\mathrm{F}_{i}=\mathrm{F}_{\max }$, including the time-dependent drop rate in intensity. The initial drop is much faster without $\mathrm{Mg}^{2+}$.

4.) DCMU: $D C M U$ immediatey raises $F_{i}$ by $\approx 23 \%$. It also raises $F_{\max }$ by $\approx 20 \%$ and $36 \%$, with and without $\mathrm{Mg}^{2+}$, respectively. Both levels remain essentially constant.

5.) DCMU + LA: DCMU effects are vitiated by LA (see \#1).

6.) DCMU $+\underline{S}_{2} \underline{O}_{4}^{2-}$ : The curve appears almost immediately like a normal dithionite curve without $\mathrm{DCMU}$, but with a slightly slower $\mathrm{F}_{o}$ rise when compared to dithionite alone; i.e., the DCMU effects are essentially masked.

7.) \#6+LA: The LA effects predominate (see \#1).

8.) $\mathrm{S}_{2} \mathrm{O}_{4}{ }^{2-}+$ DCMU: The $\mathrm{F}_{i}$ intensity is raised above the dithionite level by an additional $50 \%$ of control's $\mathrm{F}_{o}$, the same net rise as without dithionite. No change in $\mathrm{F}_{\max }$ is observed.

9.) \#8+LA: The LA effects predominate (see \#1).

10.) LA $+\underline{S}_{2} \mathrm{O}_{4}{ }^{2-}:$ Dithionite raises the fluorescence intensity by a lesser extent than for samples not pre-incubated with LA (by $31 \%$ vs. $46 \%$ in the presence of $\mathrm{Mg}^{2+}$ and by $22 \%$ vs. $55 \%$ in the absence of $\mathrm{Mg}^{2+}$ ). The $\mathrm{F}$ intensity declines subsequently at the same rate as with LA alone ( $+\mathrm{Mg}^{2+}$ data only); otherwise no effect upon curve shape is observed.

11.) LA + $\underline{\mathrm{DCMU}}$ : No effects are apparent other than a slightly slower fluorescence intensity decline.

12.) DPC: DPC merely lowers $\mathrm{F}_{\max }$ slightly.

\section{DCIP Reduction}

$\mathrm{DCIP}\left(\mathrm{E}_{\mathrm{m}}=220 \mathrm{mV}\right.$ at $\mathrm{pH}$ 7) is able to accept electrons from reduced $\mathrm{Q}_{\mathrm{B}}$ and/or other hydroquinone molecules within the quinone pool which have midpoint potentials 
near $0 \mathrm{mV}$. As it changes from blue to colorless upon reduction, DCIP reflects the progress of electron transport through PSII. It is ideal, therefore, for studying the effect of PSII inhibitors and activators. Reactions which utilize water as the ultimate source of electrons to reduce such artificial acceptors are termed Hill reactions. Both intact chloroplasts as well as fractionated thylakoids with a functioning OEC exhibit such Hill activity readily. Alternatively, artificial donors such as DPC may be used in place of water.

The particular rationale behind the DCIP reduction experiments in this study was to approach the PSII reaction center as an enzyme-like system utilizing light as a "substrate" to produce reducing equivalents, in the hope that it is amenable to the types of kinetic methods normally used to analyze inhibition of enzymes. Samples were incubated for various lengths of time with differing concentrations of linolenic acid and then illuminated. Excitation light intensity at the sample was adjusted by using neutral density filters (see Materials and Methods).

Confirming the findings of both Siegenthaler [1974] and Golbeck et al. [1980], $\mathrm{Mg}^{2+}$ was determined to have little impact on DCIP reduction rates (data not shown), unlike other divalent cations such as $\mathrm{Ca}^{2+}$ and $\mathrm{Mn}^{2+}$; therefore, bleaching experiments were performed without it.

DCIP reduction rates $( \pm \mathrm{DPC})$ increase initially with LA concentration, attributed in part to uncoupling of photophosphorylation [Cohen et al., 1969][Okamoto \& Katoh, 1977]. Golbeck, however, observed PSI activation by LA even in samples preincubated with known uncouplers, with and without DCIP present, indicating an alternative mechanism to simple uncoupling [Golbeck et al., 1980]. After a $10 \mathrm{sec}$ incubation, activity peaks at $\approx 50 \mu \mathrm{M} \mathrm{LA}$, and then declines (Figs. 20a,b). Location of the rate peak is shifted downward to $\approx 30 \mu \mathrm{M}$ LA after 5 minute incubation, indicating that the activation process is time-dependent (Figs. 22a,b).

DPC raises DCIP reduction activity over that for untreated samples, particularly at 
lower pH (Figs. 19a,b, 23a,b). In LA-treated samples, DPC appears to cause only a short-lived increase in reduction rates, again particularly at lower $\mathrm{pH}$. For example, the kinetic plots for $67 \mu \mathrm{M} \mathrm{LA}, \pm \mathrm{DPC}$, are nearly identical at $\mathrm{pH} 7.6$, whereas the same plots at pH 6 show a dramatic DPC effect (Figs. 19a,b, 24a,b). In addition to increasing rates, DPC also reduces data scatter in cases involving shorter LA incubation intervals (Figs. 20a,b, 23a,b). This can be seen particularly well in plots of reduction rates $v s$. relative light intensity according to the method of Cornish-Bowden (Figs. 21, 25). DPC does not remove scatter as effectively from samples with longer LA incubation intervals, however, making them more difficult to interpret (Figs. 22a,b, 24a,b).

Certain rate $v s . \% \mathrm{~T}$ plots show peaks in the $34 \% \mathrm{~T}$ region (Figs. 23a,b, 24b). The reason for this behavior is uncertain, but in terms of enzyme kinetics the shape of these curves suggests a homotropic light-binding effect. The peaks shift to lower LA concentrations at the longer ( $5 \mathrm{~min}$ ) incubation time, \pm DPC.

\section{$\underline{\text { P680 }}^{+}$Absorption Transients}

Fig. 26 depicts the change in $820 \mathrm{~nm}\left(\mathrm{P} 680^{+}\right)$absorption transients with time after addition of $67 \mu \mathrm{M}$ LA to everted PSII particles ( $\mathrm{pH}$ 7.6). Figs. 27a-c are derived from such absorption transients and illustrate the observable $\mathrm{P} 680^{+}$concentration $v s$. time for different concentrations of $\mathrm{LA}$ and $\mathrm{pH}$. Fig. 27a shows a monophasic decline at $\mathrm{pH} 6$ with a half time of $\approx 3-4 \mathrm{~min}$. Plots for $\mathrm{pH} 6.8$ and 7.6 (Figs. 27b,c) appear biphasic,

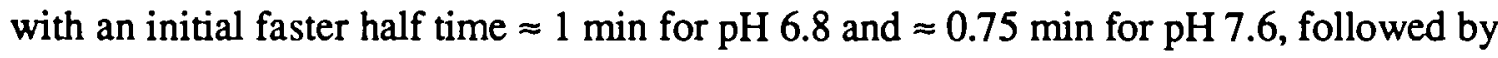
a slower $2-3$ min component for $\mathrm{pH} 6.8$ and $\approx 0$ for $\mathrm{pH}$ 7.6. The [LA] $\leq 33 \mu \mathrm{M}$ curves for $\mathrm{pH} 7.6$ appear to have an initial small plateau lasting about $1 \mathrm{~min}$ (Fig. 27c), and the plot for $[\mathrm{LA}]=13 \mu \mathrm{M}$ at $\mathrm{pH} 6$ shows an erratic behavior which is difficult to explain.

Addition of ferricyanide to samples confirmed the absence of any appreciable PSI contribution to the absorption transients. DCMU was reported by Golbeck and Warden 
[1984] to have minimal effect upon $\mathrm{P} 60^{+}$absorption transients and was not employed here.

Destruction of $\mathrm{P} 680^{+}$transients observed after treatment with LA may imply that primary charge separation is curtailed in centers inhibited with LA. Alternatively, it may mean that primary separation / recombination is occurring in inhibited centers at a rate which is outside the range of the spectrophotometer ( $3 \mu \mathrm{sec}$ compared to a Klimovproposed recombination lifetime of 2-4 nsec). 


\section{CHAPTER V}

\section{DISCUSSION}

\section{DCIP Reduction Kinetics}

In the present study, both the concentration of LA and excitation light intensity were varied in order to better relate these kinetic parameters to PSII kinetic activity, as monitored via the photoreductive bleaching of DCIP dye. Workers noticed quite early that normal Hill reactions often followed a hyperbolic velocity $v s$. light intensity relationship which could be described by equations similar to the well-known Michaelis-Menten equation for enzymes [see Lumry et al., 1954]. A kinetic approach was used by Krogmann and Jagendorf [1959] to conclude that long chain fatty acids in general inhibit the light reactions of photosynthesis as opposed to the dark reactions. In the present study, the concentration of the inhibitor linolenic acid was varied along with the "substrate" concentration, the latter by using neutral density filters to reduce excitation light intensity at the sample (see Materials and Methods). One difficulty with this approach is the combined influence of multiple modes of activity by linolenic acid (see Chapter I). The approach can still be useful, however, since not all of these effects occur simultaneously, at the same rate, or to the same extent (as will be discussed, the "primary" effects tend to overshadow the others). Nonetheless, it is not surprising that the results of the DCIP reduction experiments reflect the complexity of the LA/system interaction by being rather difficult to interpret. For example, the results from samples with LA tend to behave in a tractable and unambiguous manner only when the chloroplasts are incubated for short $(<30 \mathrm{sec}$ ) intervals and only in the presence of DPC; otherwise, scatter in the data increases, causing trends in the data to become less discernable (cf. Figs. $21 \& 25$ ). 
This scatter in the data is repeatable and is therefore not the result of statistical error, but is caused by the system deviating from Michaelis-Menten type behavior. Conclusions derived for both sets of data seem consistent with each other despite this increased scatter, however (see Data Presentation). LA is known to result in loss of manganese from the water-splitting complex [Golbeck et al., 1980][Garstka and Kaniuga, 1988]; perhaps DPC reduces scatter by bypassing the $\mathrm{OEC}$ and thus reducing its influence. Other donor-side inhibition may remain, however [Siegenthaler, 1974][Venediktov and Krivoshejeva, 1983][Golbeck and Warden, 1984][Warden and Csatorday, 1987]. Kinetic results will be discussed subsequently solely in terms of the direct-plotting technique of CornishBowden. For a good treatment of the subject the reader is referred to his text, Fundamentals of Enzyme Kinetics (Butterworths, London). Overall, the results indicate that LA-treated samples do not exhibit pure competitive-type inhibition (Fig. 21), which would be characterized by a horizontal intersection locus. This should rule out trapping inhibition at the antenna bed as a sole inhibition mode and implies that primary charge separation occurs following treatment with LA. It does not rule out trapping inhibition coincident with electron chain inhibition, however, since the 10-20 sec incubation curves appear to indicate "pure" non-competitive and/or mixed-type inhibition. Comish-Bowden points out that non-competitive inhibition is merely a special case of mixed inhibition; the former is associated with an intersection locus falling on a vertical straight line and implies equal (and concurrent) contributions from both uncompetitive and competitive sources of inhibition, whereas the mixed case is associated with either a linear locus (slope $>0$ ) or a non-linear locus and does not necessarily imply equal contributions from both inhibition types. Results also do not rule out pure uncompetitive inhibition as a contributor to the LA effect, normally expressed as a linear locus intersecting the origin. Plot intersections for 67 and $84 \mu \mathrm{M}$ LA seem to follow this course. This type of inhibition should imply reversible blockage of the electron transport chain as its source. 
Fluorescence

Magnesium Effects. $\mathrm{Mg}^{2+}$ appears to confer some short-lived resistance to the LA effect upon fluorescence. When LA is added to spinach chloroplasts which have been treated with $\mathrm{Mg}^{2+}$, both the $\mathrm{F}_{i}$ and $\mathrm{F}_{\text {max }}$ levels are initially higher than without $\mathrm{Mg}^{2+}$. In addition, both levels drop more slowly with $\mathrm{Mg}^{2+}$ present, the $\mathrm{F}_{\max }$ level especially. This $\mathrm{Mg}^{2+}$-induced time stability is also apparent when DPC, DCMU or dithionite is present in addition to the LA. The stabilizing effect of $\mathrm{Mg}^{2+}$ is particularly apparent at [LA] $<\approx 50$ $\mu \mathrm{M}$. However, the rapid drop in $\mathrm{F}$ intensity upon addition of high [LA] $(150 \mu \mathrm{M})$ to control, DCMU and dithionite-treated samples in the absence of $\mathrm{Mg}^{2+}$, as compared to the slower drop in samples with $\mathrm{Mg}^{2+}$, indicates that higher [LA] can overcome its influence quickly. The incorporation of LA into the membrane has been determined to compete favorably with precipitation with $\mathrm{Mg}^{2+}$ [Keuper, 1986]. The $\mathrm{Mg}^{2+}$ effect is probably related to its ability to promote and help to preserve stacking of the thylakoid membrane, possibly by neutralizing negative surface charges associated with stroma-exposed portions of LHC II proteins [Mullet and Arntzen, 1980][Vernotte et al., 1983][Keuper, 1986], whereas LA is known to promote destacking [Cohen et al., 1969][Shaw et al., 1976][Okamoto et al., 1977][Vernotte et al., 1983]. These repelling surface charges are fewer in number at lower $\mathrm{pH}$ and may explain the lack of an $\mathrm{F}_{\max }$ drop in the absence of added $\mathrm{Mg}^{2+}$ after LA treatment at $\mathrm{pH} 6$ (data not shown). In addition, $\mathrm{Mg}^{2+}$ is thought to increase energy coupling of LHC II to P680 [Forti, 1987]. Both destacking and LHC decoupling would decrease light-gathering and trapping efficiency.

Mediator Effects. A meaningful interpretation of the effects upon fluorescence of LA in situ with the aid of compounds such as DCMU and dithionite requires that the action of these substances within the RC be characterized and understood to a reasonable degree. The dithionite effects may be the most interesting and potentially the most revealing. As stated in the Data Presentation, two separate dithionite effects are apparent: an immediate 
and dramatic increase in $F_{\max }$ accompanied by a slower increase in $F_{i}$ leading to a diminishing $F_{v a r}$. A similar though less dramatic rise in $F_{\max }$ was observed with DCMU, which is known to stabilize the presence of $Q_{A}{ }^{-}$, but $F_{i}$ was raised only slightly in this case. The greater $\mathrm{F}_{\max }$ effect caused by dithionite may be due in part to its ability to rapidly accumulate a larger $\mathrm{Q}_{\mathrm{A}}{ }^{-}$population than $\mathrm{DCMU}$ can. If this is true, it still remains to explain the relatively slow subsequent rise of $F_{i}$. These two distinct fluorescence effects caused by dithionite may indicate the reduction of two distinct PSII quencher/acceptors, one at a much faster rate than the other. This suggests the existence of another electron acceptor prior to $\mathrm{Q}_{\mathrm{A}}$, either as part of the transport chain or at least accessible to it. This hypothetical acceptor will henceforth be designated "R." Data supporting the existence of extra PSII acceptors, the most prominent three being designated $X_{a}, Q_{2}$, and $U$ by various workers, has been reported by many groups [Black et al., 1986][Mathis and Rutherford, 1987]. Like the plastoquinone $Q_{A}, \Re$ may behave as a fluorescence quencher in its oxidized state.

Results indicate that the DCMU-mediated process is complete in less than the 10$20 \mathrm{sec}$ interval between reagent addition and fluorescence measurement achieved in this work. As stated, the lower $\mathrm{F}_{\max }$ produced by DCMU compared to dithionite could be attributed to the latter's ability to accumulate a lower steady-state concentration of the photoactive quencher $Q_{A}$ by chemically reducing it to $Q_{A}{ }^{-}$with high efficiency. That DCMU and dithionite exhibit different effects is reasonable considering the completely different mechanism associated with each substance. Dithionite is a strong reductant, whereas DCMU is an inhibitor which is thought to shift the equilibrium for the process $Q_{A}^{-} Q_{B} \Leftrightarrow Q_{A} Q_{B}^{-}$to the left by displacing of $Q_{B}$ from its binding site on D1 [Lavergne, 1982]. The comparatively lower $\left[Q_{A}\right]$ achieved with dithionite would also explain the smaller $\mathrm{F}_{v a r}$ seen with this compound. Therefore, the relatively small $\mathrm{Q}_{\mathrm{A}}$ population associated with DCMU and dithionite treatment, whether separate or in combination, 
probably explains the high $\mathrm{F}_{\max }$ levels observed with these agents when compared to untreated samples. In addition, the low light intensity used in this study allowed $\mathrm{F}_{\max }$ to remain high by preventing the photoaccumulation of pheophytin, even in the presence of the artificial donor DPC [ $c f$. Renger et al., 1983 and Klimov et al., 1985]. Lavergne, using closely spaced saturating flashes to poise the OEC at a low-potential S state, observed an increase in $\mathrm{F}_{\max }$ in DCMU-treated samples irradiated with high light intensity. He attributed this to decreased back reaction of $\mathrm{Q}_{\mathrm{A}}{ }^{-}$with the donor side of PSII [Lavergne, 1982]. Low concentrations of hydroxylamine, which is known to stabilize the lowpotential $\mathrm{S}_{\mathrm{o}}$ state of the OEC, was reported to raise $\mathrm{F}_{\max }$ for this reason in DCMU-treated samples irradiated at low light intensity [Bennoun, 1970]. Dithionite may produce a similar effect by destabilizing the $S_{2} \& S_{3}$ states. Another contribution to the rise in $F_{\max }$ may be enhanced electron flow through the water splitting system [Klimov et al., 1985] Thus, when dithionite was added to samples treated with LA $(150 \mu \mathrm{M})$ or LA plus DCMU, which should inhibit any direct quinoidal influence (see below), the fluorescence intensity was raised by a lesser amount than in the control, presumably revealing the enhanced donor-side contribution $\left(\approx 40 \%\right.$ of the total $\mathrm{F}_{\max }$ rise). No comparable rise in fluorescence intensity was observed with DCMU

Neither dithionite nor DCMU alone stops all secondary electron flow at the concentrations used in the experiment, as demonstrated by the existence of a residual induction phase $\left(\equiv F_{v a r}\right.$ ), especially apparent with DCMU (Fig. 11). Others have attributed the residual induction phase with DCMU to centers which are resistant to the inhibitor, known as "B-type" centers [Lavergne, 1982][Black et al., 1986].5 This interpretation is yet another notable controversy concerning PSII, and may have been fueled, as Mathis and Rutherford point out [1987], by the hope that higher plants conform

5 The complex subject of PSII heterogeneity is covered in detail in reviews by Black et al., 1986 and Mathis \& Rutherford, 1987. 
to the bacterial model derived recently for crystallized reaction centers. (The same rationale is detectable in resistance to proposals of an additional acceptor in PSII). Nonetheless, assuming for the moment that $\Re$ exists in all centers, chemical reduction of $\Re$ could explain to the slow rise in $F_{i}$ and complementary diminishing of $F_{v a r}$ observed with dithionite. The reduction of $\Re$ may occur more slowly than $\mathrm{Q}_{\mathrm{A}}$ because $\Re$ 's binding site is sequestered to a greater extent from the external redox environment, possibly by being located further within the thylakoid membrane. Since the area above the dithionite rise curve represents only about $5 \%$ of the original area seen above the control curve, however, $\Re$ is likely not a member of the main transport chain, but may normally be accessed by it when $Q_{A}$ has been photochemically reduced. In fact, in the absence of direct chemical reduction $\Re$ may only be accessible via $\mathrm{Q}_{A}{ }^{-}$. This would allow for the small $F_{i}$ rise seen with DCMU. Velthuys and Amesz invoked an analogous indirect reduction process between the quinone pool and "Q" to explain certain aspects of their experimental results with dithionite [Velthuys and Amesz, 1973]. The comparatively minor extent of this $F_{i}$ rise implies that $\Re$ is not reduced easily by this pathway, possibly due to its low potential between $\mathrm{Ph}(-610 \mathrm{mV})$ and $\mathrm{Q}_{\mathrm{A}}(-130 \mathrm{mV})$. Its equilibration with dithionite would place $\Re$ at above $-420 \mathrm{mV}$. Renger et al. [1983] estimated the potential of a proposed additional acceptor (designated "A") to be $-300 \mathrm{mV}$. Alternatively, $\Re$ could represent a heterogeneity present in only a fraction of PSII centers. Such a minor (5\%) component of fluorescence induction appears inconsistent with literature reports from workers advocating heterogeneity, however [Black et al., 1986]. While the above model accounts for the masking of the DCMU effect upon subsequent addition of dithionite, it is unclear why $F_{i}$ is raised more slowly in this case, or why DCMU raises $F_{i}$ further when added after dithionite (see fluorescence data presentation summary above). 
Model

Results of this and other selected fluorescence studies are consistent with the reversible blockage of secondary electron flow between $\mathrm{Ph}$ and $\mathrm{Q}_{\mathrm{A}}$ by linolenic acid as postulated by Golbeck and Warden [Golbeck and Warden, 1984]. The exact nature of the "blockage" remains undefined, but may include either a reversible alteration of the D1 and/or D2 polypeptides in the vicinity of the $Q_{A}$ binding sites, or "displacement" of $Q_{A}$ from one or both of these sites. An LA-induced abolition of absorbance changes at 320 $\mathrm{nm}\left(\mathrm{Q}_{\mathrm{A}} / \mathrm{Q}_{\mathrm{A}}{ }^{-}\right)$and electron spin resonance associated with $\mathrm{Q}_{\mathrm{A}}{ }^{-} \mid \mathrm{Fe}$ supports this view [Warden \& Csatorday, 1987]. In addition, fluorescence decay measurements taken from LA-treated samples by S. Tabbutt, J. H. Golbeck and K. Sauer [personal communication from J.H. Golbeck] reveal similar decay components as in DCMU-treated samples (Appendix B). The slow component was found to increase in lifetime and yield in both cases, with the only difference being that the DCMU treated sample required enough light intensity to transition from $\mathrm{F}_{o}$ to $\mathrm{F}_{\max }$ beforehand whereas LA exhibited $\mathrm{F}_{\max }$ results irrespective of light intensity. This indicates that although the mechanism of LA inhibition is probably different than DCMU, the effects upon fluorescence are similar and support a blockage of the transport chain at different locations by each compound. Such a blockage would explain the ability of LA to vitiate the fluorescence effects of both dithionite and DCMU-treated samples. If this blockage also encompassed the $\mathrm{Q}_{\mathrm{A}}$-mediated path to $\Re$ proposed above, then LA inhibition would be expected to prevent the $F_{i}$ rise normally associated with DCMU addition, but not prevent its rise upon dithionite addition since the latter is able to reduce $\Re$ directly (Figs. $16 \& 18$ ).

Implicit in the above fluorescence model is that primary charge recombination between $\mathrm{Ph}^{-}$and $\mathrm{P}_{680^{+}}$is the major source of $\mathrm{F}_{\max }$ in centers closed with dithionite, DCMU or LA. The diminished $\mathrm{P} 60^{+}$absorption changes observed in LA-treated PSII particles can be interpreted to support the presence of primary charge separation in centers 
closed with LA, as can the DCIP bleaching results (see Data Presentation). In addition, an ESR signal attributed to charge recombination has been observed in dithionite-treated centers inhibited with LA (below). The potential dependence of $F_{\max }$ found by Warden \& Csatorday upon titrating the $\mathrm{Ph} / \mathrm{Ph}^{-}$pair in the presence of LA also supports the view that significant primary charge separation occurs in centers closed with LA and that $F_{\text {max }}$ is intimately related to this step [Warden and Csatorday, 1987].

However, this study cannot determine whether or not the rise of $F_{i}$ to $F_{\max }$ in centers closed with LA is not due at least in part to trapping inhibition, such as by decoupling of the antennae from the reaction center. This would allow a portion of the excitation energy to be re-emitted promptly by bypassing the primary charge separation step and is consistent with the view held by Holzwarth and others regarding closed centers in general. Indeed, results of the DCIP bleaching kinetics here indicate that some trapping inhibition may be occurring in LA treated centers. One difficulty with this conclusion, however, is the similarity of the fluorescence decay components from both LA and DCMU-treated samples (mentioned above) despite the fact that the activity of DCMU is not known to extend to the antenna bed.

Recently Schatz et al. [1987] has detected an absorbance change both in open centers (lifetime $\approx 100 \mathrm{psec}$ ) and in those closed with dithionite (lifetime $\approx 200 \mathrm{psec}$ ) with difference spectra indicating that exciton trapping leading to primary charge separation may be reduced by $50-70 \%$ in closed centers. The Schatz group also detected a 1.6-1.8 nsec absorbance component in closed centers. Unfortunately, the difference spectrum offered by Schatz for this component encompassed only the very limited spectral region of 620$700 \mathrm{~nm}$, but it appears that it could contain a significant $\mathrm{Ph}-/ \mathrm{Ph}$ contribution based upon comparison with the difference spectrum reported by Klimov [Klimov et al., 1977]. The reported lifetime for this component was $\approx 2 \mathrm{nsec}$ - close to that predicted by Klimov for charge recombination - implying that primary charge separation / recombination may be 
occurring to a greater extent than Schatz's estimate (although the possibility of diminished charge separation in closed centers remains inconsistent with Klimov's original hypothesis).

Both Warden and Csatorday [1987] and the Tabbutt et al. [personal communication from J.H. Golbeck] have used EPR to analyze various signal changes induced by LA. They discovered that a radical pair spin-polarized triplet (RPT), proposed to originate via charge recombination between $\mathrm{P}_{680^{+}}$and $\mathrm{Ph}^{-}$, is nonexistent in samples treated with LA alone, but appears in dithionite treated samples $\pm \mathrm{LA}$. These results were unexpected since the type of blockage envisioned with $\mathrm{LA}$ (between $\mathrm{Ph}$ and $\mathrm{Q}_{\mathrm{A}}$ ) was expected to generate such a signal by forcing primary recombination. At first one might assume that there exists a low-temperature donor to $\mathrm{P} 680^{+}$which prevents recombination by allowing photoaccumulation of $\mathrm{Ph}^{-}$; however, this does not explain the dithionite dependence since it is unlikely that dithionite would disable such a donor. Instead, both groups suggested as an explanation the possibility of an additional acceptor as a sequential member of the pathway between $\mathrm{Ph}$ and $\mathrm{Q}_{\mathrm{A}}$, as proposed earlier by Evans [1985] (among others). However, the Tabbutt group points out the inconsistency of an instantaneous fluorescence rise if this is true since the fluorescence should remain low until this acceptor becomes reduced upon illumination. As an alternative they suggested that coulombic interaction of $\mathrm{Q}_{\mathrm{A}}{ }^{-}$upon $\mathrm{Ph}$ could facilitate recombination and thereby explain the need for the reductant dithionite. These two components have been reported to be separated by only $0.8-1.1 \mathrm{~nm}$ in normal centers [Klimov et al., 1985]. Reduction of $\Re$ could conceivably achieve the same effect, however, particularly if its relatively sequestered environment (above) allowed a location even closer to $\mathrm{Ph}$ than $\mathrm{Q}_{\mathrm{A}}$. In addition, if the pathway to $R$ is altered or blocked by LA as proposed above the RPT would likewise not be expected in the absence of dithionite.

The chemical composition of $\Re$ cannot be identified within the context of this 
study. It is probably not a quinone since extraction studies indicate sufficient quinone on the acceptor side of PSII to only accommodate $Q_{A} \& Q_{B}$ [De Vitry et al., 1986]. It is interesting to note that Cox identified a carotenoid, presumably in close proximity to $\mathrm{Ph}$, which plays no direct role in electron transfer but whose presence is necessary in order to observe the $550 \mathrm{~nm}$ bandshift attributed to $\mathrm{Ph}$ [Cox and Bendall, 1974]. 


\section{CHAPTER VI}

\section{CONCLUSION}

This study supports the presence of two predominant modes of inhibition by LA within PSII: primary inhibition, i.e., inhibition of the process of energy trapping and/or primary charge separation, and donor side inhibition. In addition, the ability of $\mathrm{Mg}^{2+}$ to delay a drop in fluorescence intensity normally associated with thylakoid exposure to LA was explained by the ability of this cation to confer resistance to LA-induced destacking of thylakoid membranes.

Donor side inhibition occurs most likely at the $\mathrm{OEC}$ and was evidenced by the nonMichaelis-Menten PSI response upon exposure to higher concentrations of LA for longer than $30 \mathrm{sec}$, and diminished in the presence of the artificial donor DPC.

Primary inhibition is the more fundamental and controversial type of inhibition process caused by LA. An LA-induced blockage of the electron transport chain between $\mathrm{Ph}$ and $\mathrm{Q}_{\mathrm{A}}$ is supported by DCIP reduction kinetics, flash-induced $\mathrm{P} 680^{+}$absorption kinetics, and steady-state fluorescence experiments. This type of inhibition implies that primary charge separation still occurs in centers closed with dithionite, DCMU or LA, and that the predominant source of $\mathrm{F}_{\max }$ is primary charge recombination - a view promulgated by Klimov and others. The DCIP kinetic plots of LA-treated chloroplasts revealed evidence of non-competitive and/or pure uncompetitive types of LA inhibition, which could encompass inhibition of the transport chain. Diminished $\mathrm{P} 60^{+}$absorption transients in the presence of LA may have resulted from fast re-reduction of $\mathrm{P} 680^{+}$ following charge separation / recombination. Also, fluorescence rise curves lacked any visible $F_{v a r}$ in the presence of $L A$, implying isolation of the quencher $Q_{A}$ from the electron 
transport chain via the type of blockage described above.

An alternative view, promulgated by Holzwarth and others, holds that primary charge separation is negligible in closed centers. According to this view, which is based largely upon picosecond fluorescence decay experiments, primary inhibition resulting in center closure allows energy transfer possibly only as far as the shallow trap P680, followed by re-emission. Results of this study cannot rule out this type of inhibition by LA, possibly via isolation of the antennae bed from P680. The DCIP kinetic plots showed evidence of competitive and mixed inhibition by LA, which can accomodate Holzwarth's model. Diminution of $\mathrm{P} 60^{+}$transient absorption could have been caused by a lack of energy trapping, which would by necessity rule out any subsequent charge separation. Finally, the lack of $F_{v a r}$ could have resulted from emission in lieu of charge separation.

This author considers it likely that primary charge separation occurs to a significant degree in PSII centers closed with LA, but at the same time leaves open the possibility that it may accompany trapping inhibition. A model was constructed in which the source of a significant percentage of the variable fluorescence emission from closed centers was identified as delayed emission following charge recombination, based upon the separate and combined effects of LA, DCMU and dithionite upon steady-state fluorescence. This model supported the presence of an additional electron acceptor present at room temperature and below. This acceptor, designated "R," is probably not a sequential member of the transport chain but may be accessible to the chain unless blocked, such as by LA. Evidence was shown indicating that $R$ is accessible to equilibration with dithionite, and $\Re^{-}$was proposed to exert a coulombic effect upon $\mathrm{Ph}$, thereby affecting the degree of primary charge recombination. This author is aware that designating another acceptor tends to complicate the issue and he does not rule out the possibility that $\Re$ is related to one of the several acceptors already proposed.

Many researchers doubt the prospects of a crystallized PSII reaction center from 
higher plants becoming available in the very near future. In the absence of $x$-ray analysis, arguments for or against the existence of $\Re$, or of any of the other proposed additional acceptors, may remain rather speculative. Future progress will require careful spectroscopic work. PSII particles, rather than the whole chloroplasts used in this study, may be more appropriate for study into this matter because of the overall complexity of intact systems, including the multiplicity of potential inhibition sites in whole chloroplasts. Techniques must be developed to separate heterogeneous PSII center types, if possible, to resolve the extent that heterogeneity plays regarding this question. In addition, studies should be continued with the methyl ester of linolenic acid in order to better determine the influence of pH upon its activity [Vernotte et al., 1983], and with ADRY reagents such as 2-(3-chloro-4-trifluoromethyl)-anilino-3,5-dinitrothiophene ("ANT 2p") which deactivate higher $\mathrm{S}$ states in particles with intact donor systems. Electron spin resonance spectroscopy (ESR) should also be used to monitor the effect of LA upon PSII acceptors, $\mathrm{Q}_{\mathrm{A}}$ and $\mathrm{Q}_{\mathrm{B}}$ in particular. 


\section{REFERENCES}

Anderson, J. (1987) in Photosynthesis, (Jan Amesz, ed.) Elsevier Science Publishers, Amsterdam, The Netherlands, pp. 273-297.

Armstrong, J. (1964) Biochim. Biophys. Acta 86, pp. 194-197.

Arnon, D.I. (1963) Plant Physiol. 24, pp. 1-15.

Bennoun, P. (1970) Biochim. Biophys. Acta 216, pp. 357-363.

Black, M., Brearly, T. and Horton, P. (1986) Photosynth. Res. 8, pp. 193-207.

Breton, J. (1982) FEBS Lett. 147, pp. 16-20.

Breton, J. (1983) FEBS Lett. 159, pp. 1-5.

Butler, W. (1978) Annu. Rev. Plant Physiol. 29, pp. 345-378.

Butler, W. and Strasser, R. (1977) Proc. Natl. Acad. Sci. USA 74, pp. 3382-3385.

Cho, F., and Govindgee (1970) Biochim. Biophys. Acta 205, pp. 371-378.

Clark, W. (1960) Oxidation-Reduction Potentials of Organic Systems, Williams and Wilkins, Baltimore, p.403.

Cohen, W., Nathanson, B., White, J. and Brody, M. (1969) Arch. Biochem Biophys. 135, pp. 21-27.

Cornish-Bowden, A. (1979) Fundamentals of Enzyme Kinetics, Butterworths \& Co., London.

Cox, R. and Bendall, D. (1974) Biochim. Biophys. Acta 347, pp. 49-59.

Cramer, W. and Butler, W. (1969) Biochim. Biophys. Acta 172, pp. 503-510.

Debus, R., Barry, B., Sithole, I., Babcock, G. and McIntosh, L. (1988) Biochemistry 27, pp. 9071-9074.

Delosme, R. (1967) Biochim. Biophys. Acta 143, pp. 108-128.

De Vitry, C., Carles, C. and Diner, B. (1986) FEBS Lett. 196, pp. 203-206.

Evans, M., Atkinson, Y. and Ford, R. (1985) Biochim. Biophys. Acta 806, pp. 247-254.

Flexser, L., Hammett, L. and Dingwall, A. (1935) J. Am. Chem. Soc. 57, p. 2103. 
Forti, G. (1987) in Photosynthesis, (Jan Amesz, ed.) Elsevier Science Publishers, Amsterdam, The Netherlands, pp. 1-19.

Garstka, M. and Kaniuga, Z. (1988) FEBS Lett. 232, pp. 372-376.

Geacintov, N., Breton, J. and Knox, R. (1986) Advances in Photosynthesis Research, Vol. 10, pp. 233-242, Martinus Nijhoff, Dordrecht.

Golbeck, J. and Kok, B. (1979) Biochim. Biophys. Acta 547, pp. 347-360.

Golbeck, J., Martin, I. and Fowler, C. (1980) Plant Physiol. 65, pp. 707-713.

Golbeck, J. and Warden, J. (1984) Biochim. Biophys. Acta 767, pp. 263-271.

Green, B., Karukstis, K. and Sauer, K. (1984) Biochim. Biophys. Acta 767, pp. 574581.

Haehnel, W., Holzwarth, A. and Wendler, J. (1983) Photochem. Photobiol. 37, pp. 435443.

Haehnel, W., Nairn, J., Reisberg, P. and Sauer, K. (1982) Biochim. Biophys. Acta 680, pp. 161-173.

Hodges, M. and Moya, I. (1986) Biochim. Biophys. Acta, pp. 193-202.

Hodges, M. and Moya, I. (1987) Biochim. Biophys. Acta 892, pp. 42-47.

Holzwarth, A. (1986) Encyclopedia of Plant Physiology, New Series Vol. 19 (Staehelin, A. and Arntzen, C.J., eds.), pp. 299-309, Springer Verlag, Berlin.

Holzwarth, A., Wendler, J. and Haehnel, W. (1985) Biochim. Biophys. Acta 807, pp.155-167.

Kamen, M. (1963) Primary Processes in Photosynthesis, Academic Press, New York.

Ke, B., Hawkridge, F. and Sahu, S. (1976) Proc. Natl. Acad. Sci. U.S. 73, pp. 22112215.

Keuper, H. (1986) personal communication to J. H. Golbeck.

Klimov, V., Allakhverdiev, S. and Pashchenko, V. (1978) Doklady Akademii Nauk SSSR 242, pp. 1204-1207.

Klimov, V., Klevanik, A., Shuvalov, V. and Krasnovsky, A. (1977) FEBS Lett. 82, pp. 183-186.

Klimov, V., Shuvalov, V. and Heber, U. (1985) Biochim. Biophys. Acta 809, pp. 345350.

Kramer, H. and Mathis, P. (1980) Biochim. Biophys. Acta 593, pp. 319-329. 
Krogmann, D. and Jagendorf, A. (1959) Arch. Biochem. Biophys. 80, pp. 421-430.

Lavergne, J. (1982) Biochim. Biophys. Acta 682, pp. 345-353.

Lumry, R., Spikes, J. and Eyring, H. (1954) Annual Review of Plant Physiology 5 , pp. 271-340.

Mathis,P. (1984) in Advances in Photosynthetic Research, (Sybesma, C., ed.) Nijhoff / Junk, The Hague, pp. 155-158.

Mathis, P. and Rutherford, A. (1987) in Photosynthesis, (Jan Amesz, ed.) Elsevier Science Publishers, Amsterdam, The Netherlands, pp. 63-96.

Mathis, P. and Schenck, C. (1982) In: Solar Energy Research and Development in Photochemical, Photoelectrochemical and Photobiological Processes, (Commission European Communities, ed.) Reidel, Dordrecht, pp. 129-133.

Moya, I., Hodges, M. and Barbet, J. -C. (1986) FEBS Lett. 198, pp. 256-262.

Moya, I. and Garcia, R. (1983) Biochim. Biophys. Acta 722, pp. 480-491.

Mullet, J and Arntzen, C. (1980) Biochim. Biophys. Acta 589, pp. 100-117.

Murphy, D. (1986) Biochim. Biophys. Acta 864, pp. 33-94.

Neubauer, C. and Schreiber, U. (1987) Z. Naturforsch. 42c, pp. 1246-1254.

Okamoto, T. and Katoh, S. (1977) Plant \& Cell Physiol. 18, pp. 539-550.

Okamoto, T., Katoh, S. and Murakami, S. (1977) Plant \& Cell Physiol. 18, pp. 551-560.

Renger, G., Koike, H. and Inoue, Y. (1983) FEBS Lett. 163, pp. 89-93.

Schatz, G., Brock, H. and Holzwarth, A. (1987) Proc. Natl. Acad. Sci. USA 84, pp. 8414-8418.

Schreiber, U. and Neubauer, C. (1987) Z. Naturforsch. 42c, pp. 1255-1264.

Shaw, A., Anderson, R. and McCarty, R. (1976) Plant Physiol. 57, pp. 724-729.

Siegenthaler, P. (1974) FEBS Lett. 39, pp. 337-340.

Spikes, J., Lumry, R. and Rieske, J. (1955) Arch. Biochem. Biophys. 55, p. 25.

Strehler, B. and Arnold, W. (1951) J. Gen. Physiol. 34, pp. 809-820.

Trebst, A. (1987) Z. Naturforsch. 42c, pp. 742-750.

Van Gorkom, H. (1974) Biochim. Biophys. Acta 347, pp. 439-442. 
Van Dorssen, R., Plijter, J., Dekker, J., den Ouden, A., Amesz, J. and van Gorkom, H. (1987) Biochim. Biophys. Acta 890, pp. 134-143.

Velthuys, B. and Amesz, J. (1973) Biochim. Biophys. Acta 325, pp. 126-137.

Velthuys, B. and Amesz, J. (1974) Biochim. Biophys. Acta 333, pp. 85-94.

Venediktov, P. and Krivoshejeva, A. (1983) Planta 159, pp. 411-414.

Vernotte, C., Solis, C., Moya, I., Maison, B., Briantis, J., Arris, B. and Johnannin, G. (1983) Biochim. Biophys. Acta 725, pp. 376-383.

Warden, J. and Csatorday, K. (1987) Biochim. Biophys. Acta 890, pp. 215-223. 
APPENDDX A

FIGURES 6 THROUGH 27 


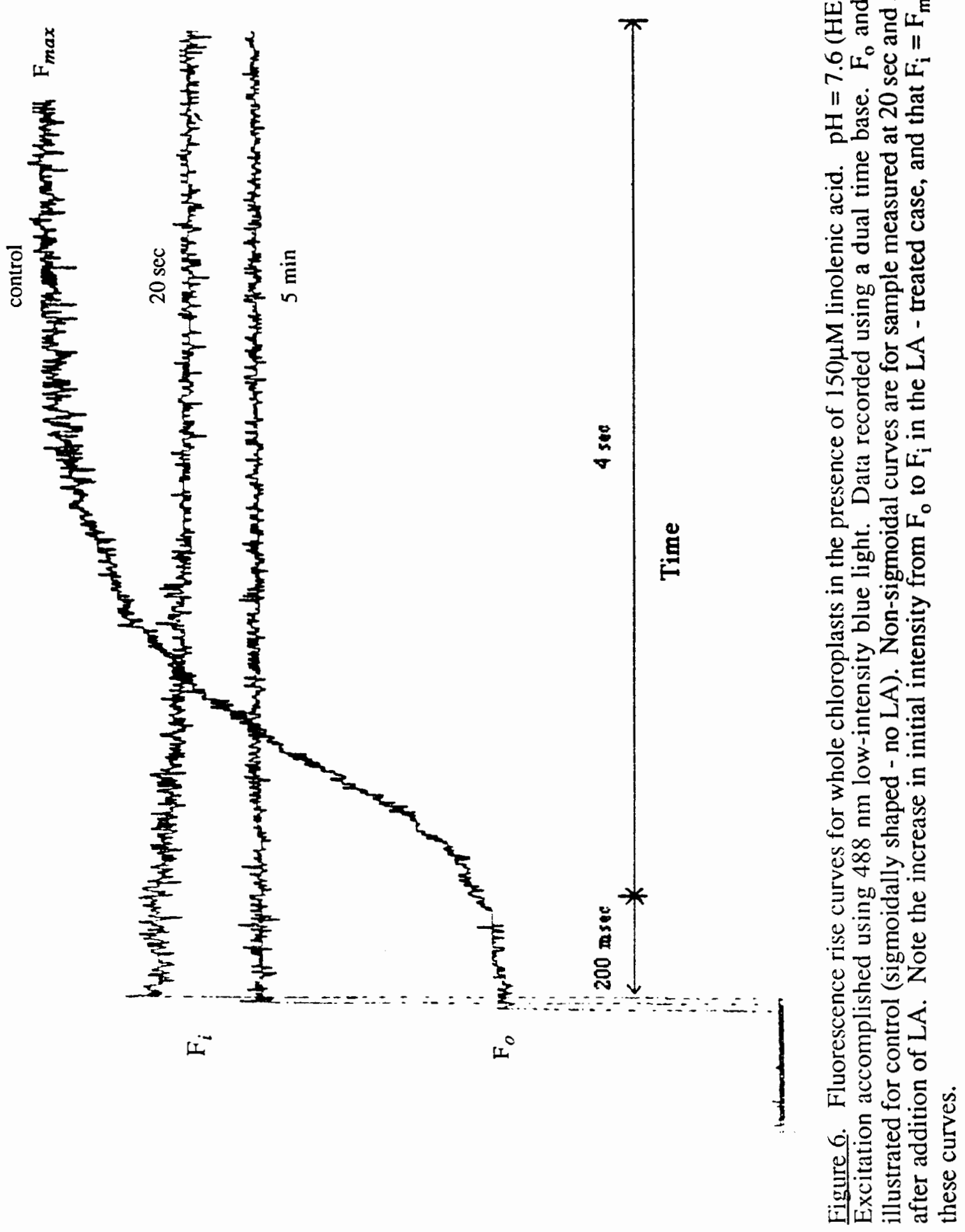

穴

红 n

도르도

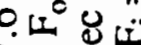
II ن웅 I 过 을 政 $\pi$ 要 政 구의 든 政

政 政 年 을을 단.

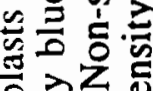
․․․ 列安 일 능 政

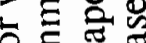
的 西 드정

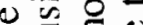
作 政

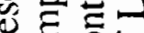
政 压

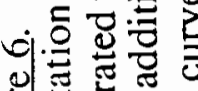
댄. 


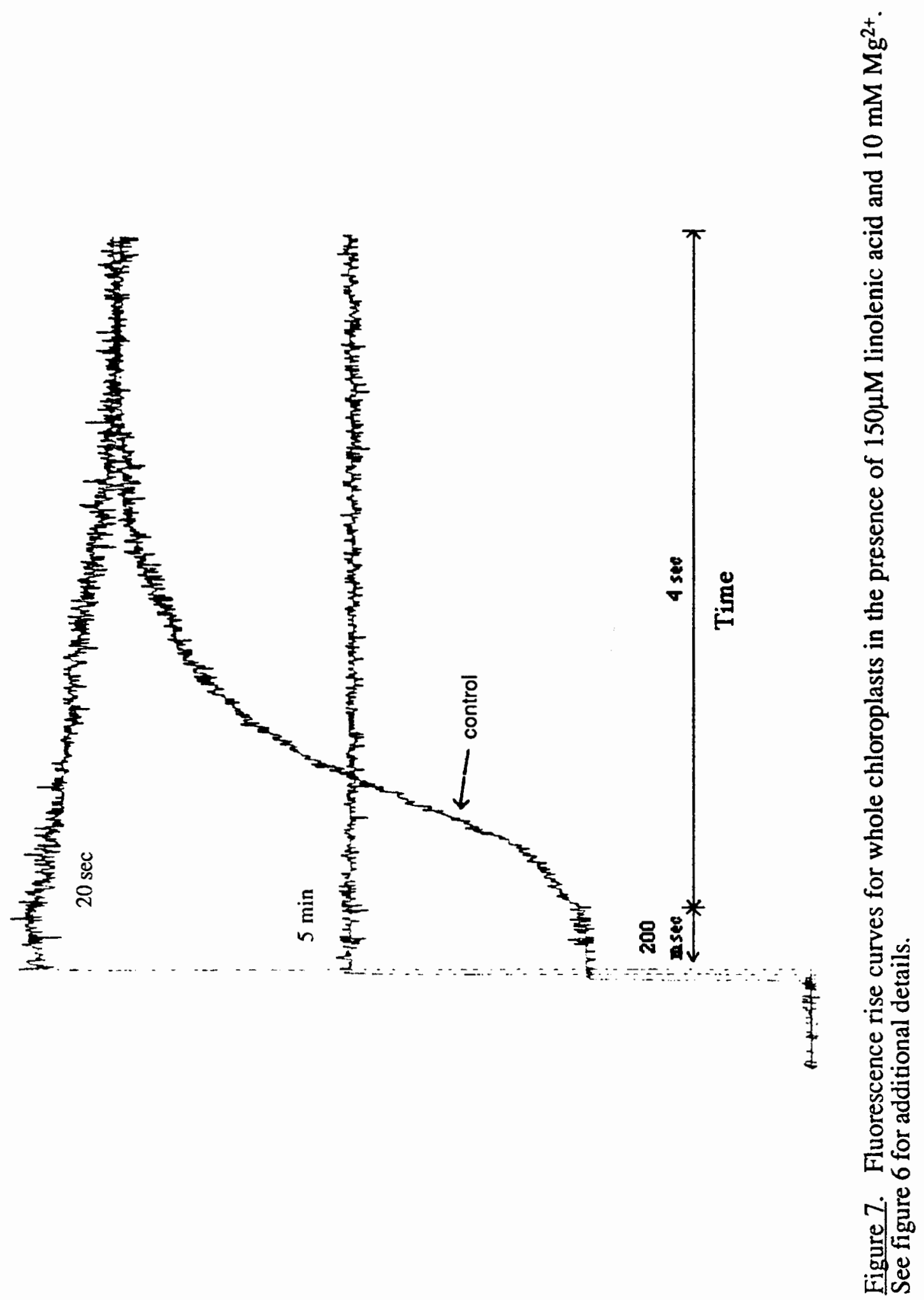




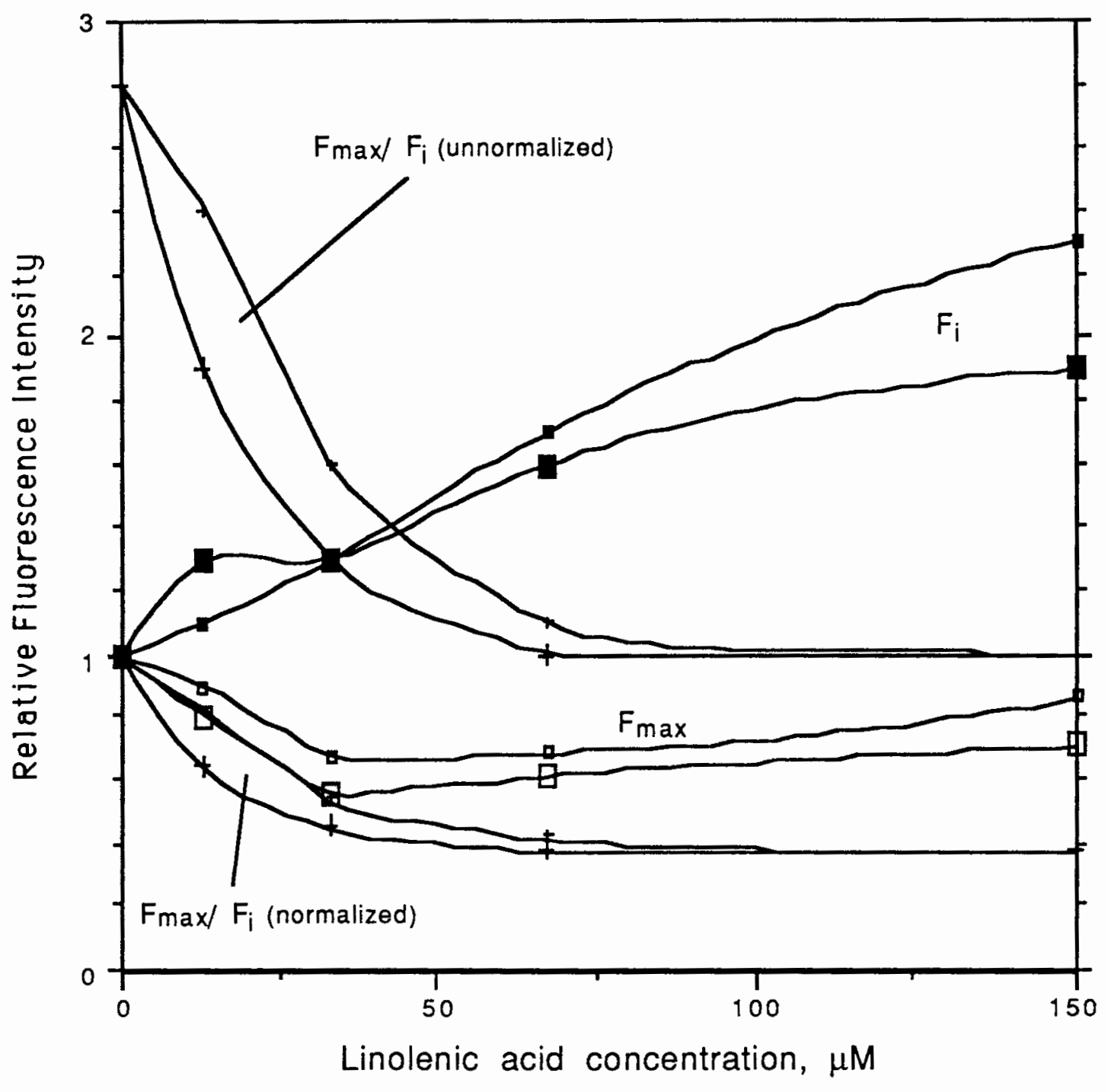

Figure 8. Relative fluorescence intensity vs. linolenic acid concentration. Smaller symbols represent a 20 second LA incubation period, larger symbols a 5 minute incubation, $\mathrm{pH}$ 7.6. 
을 㟔

$\sum_{\text {¿ }}^{+\infty}$

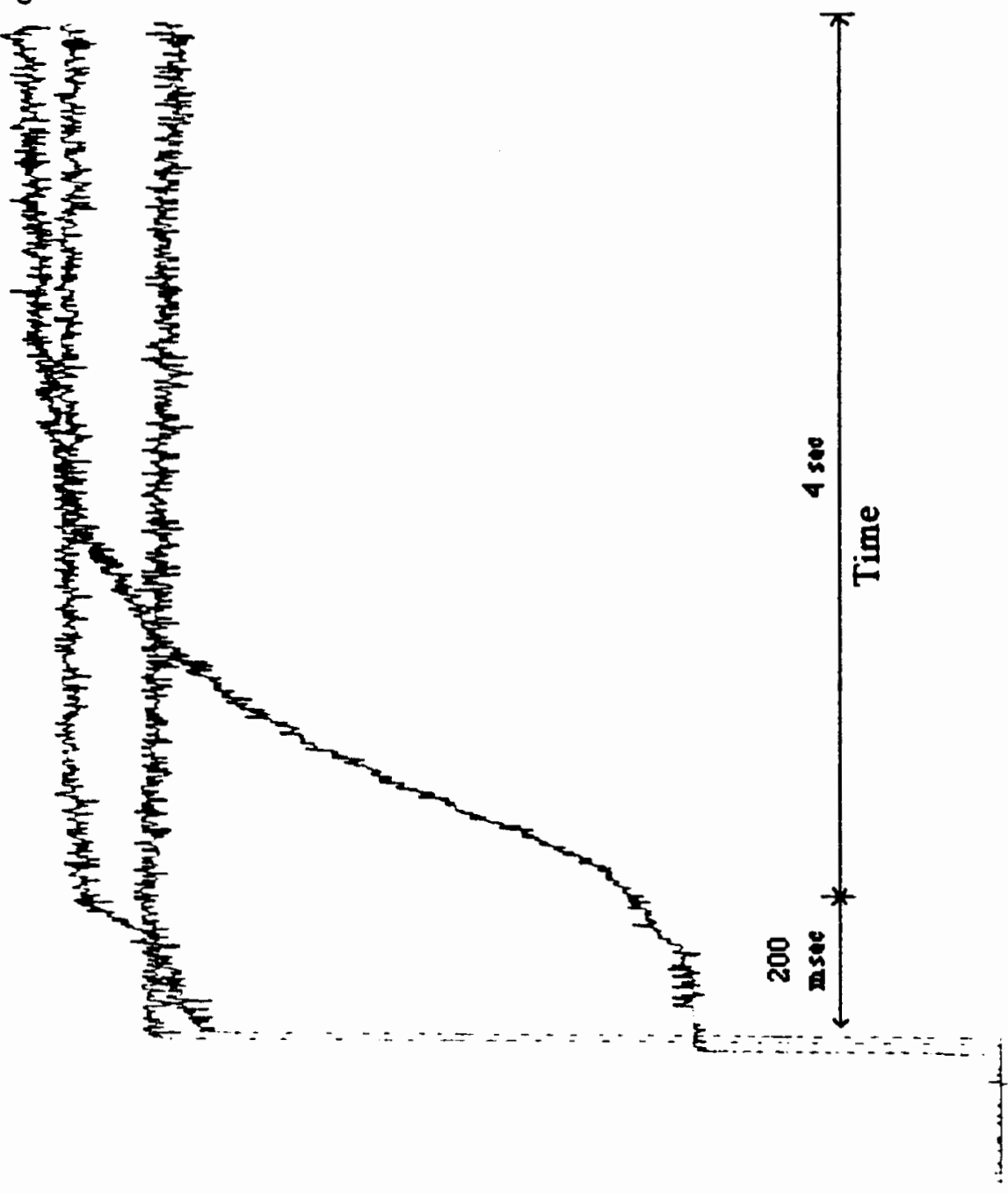

్ㅗㅇ

융

.

응

$\sum$

5

¿

巳ั

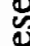

立

$\stackrel{Ð}{\Xi}$

.

号

$\frac{0}{3}$

s

s

5

점

ه

픙

음

유

은

压它

일 


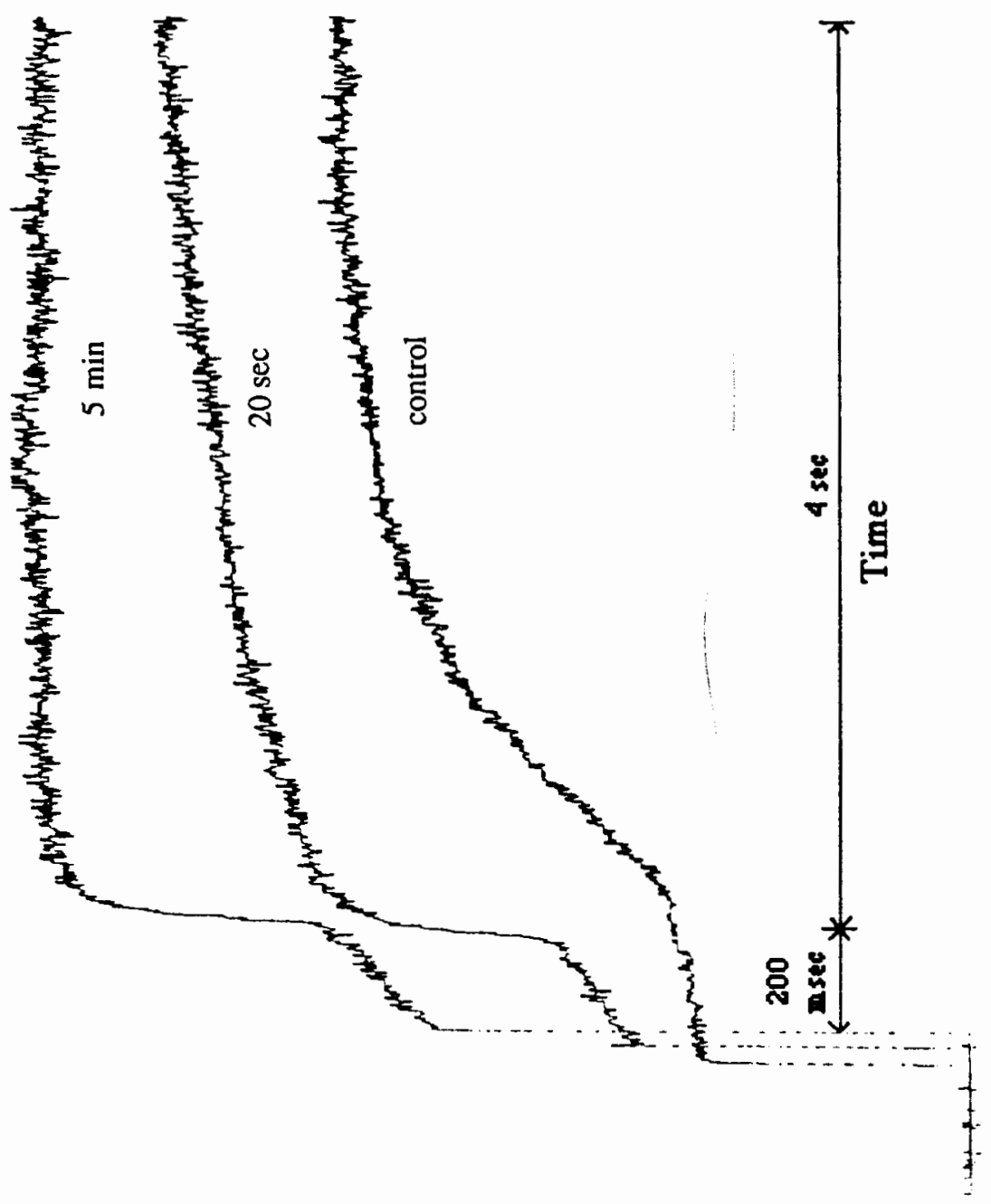

ن

.

응

品

$\sum 0$

궁엉

Љ

पै

Ð

过

空

.

$\Xi 2$

कิ $x^{\circ}$

घ

미봅

응

恄导

สำ

品:

흥.

홍

공.

品

施

눈

远

赔焉

\&

음

渮

造说

速

总

$4 \mathrm{~V}$

위는

의

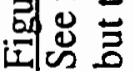




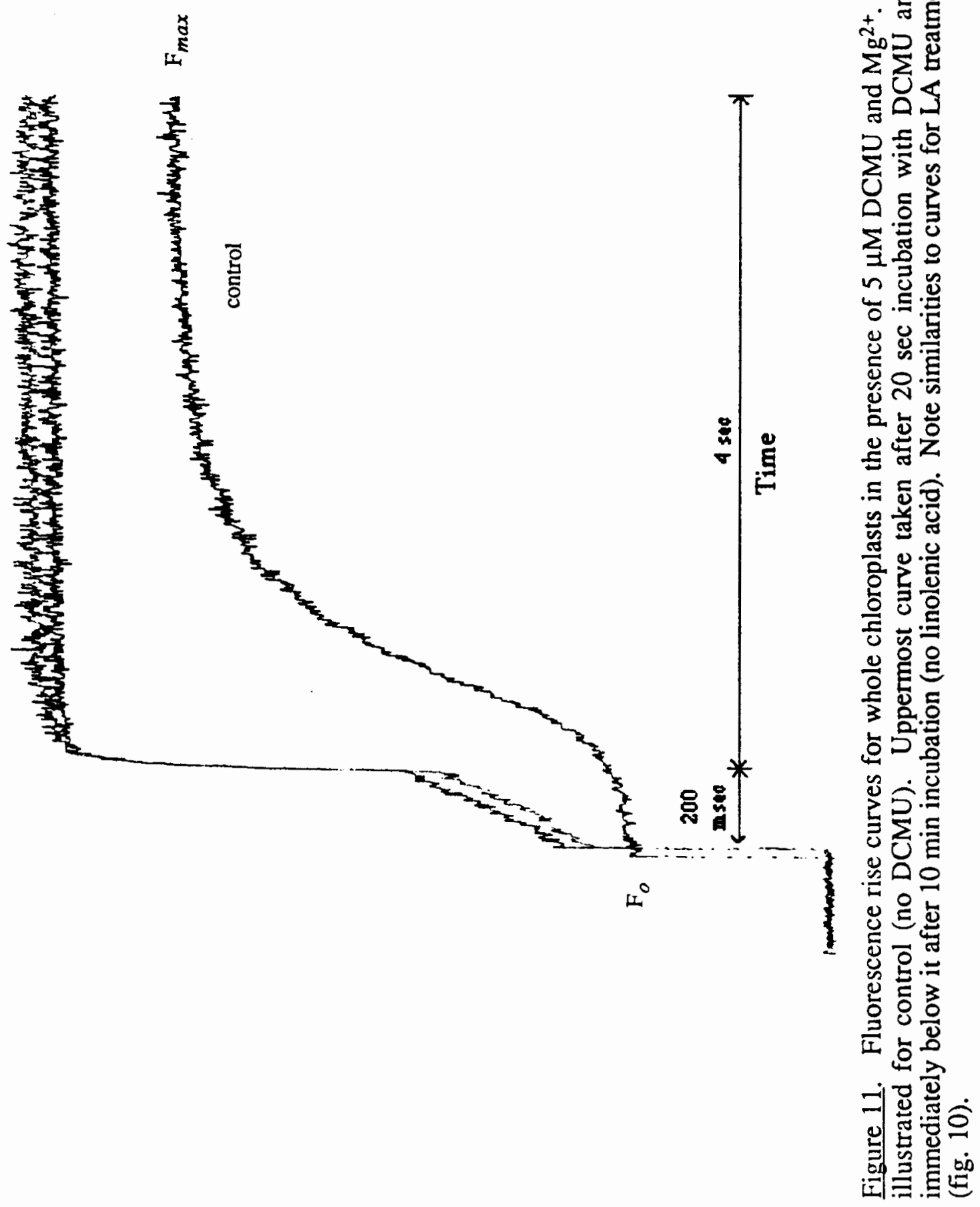




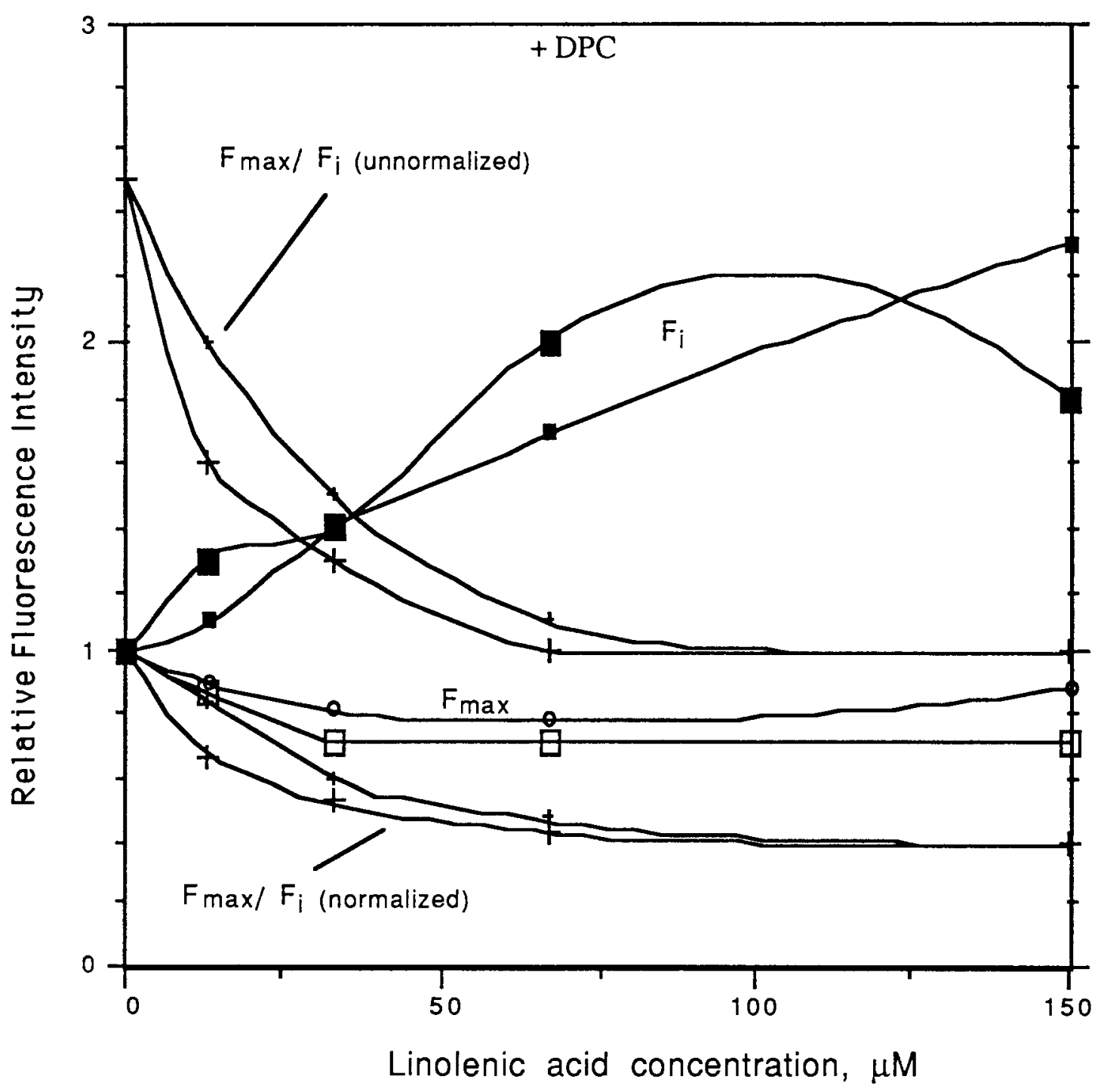

Figure 12. Relative fluorescence intensity vs. linolenic acid concentration in the presence of $500 \mu \mathrm{M}$ DPC. Smaller symbols represent a $20 \mathrm{sec}$ LA incubation period, larger symbols a $5 \mathrm{~min}$ incubation, $\mathrm{pH} 7.6$ (HEPES). See fig. 6 for additional details. 

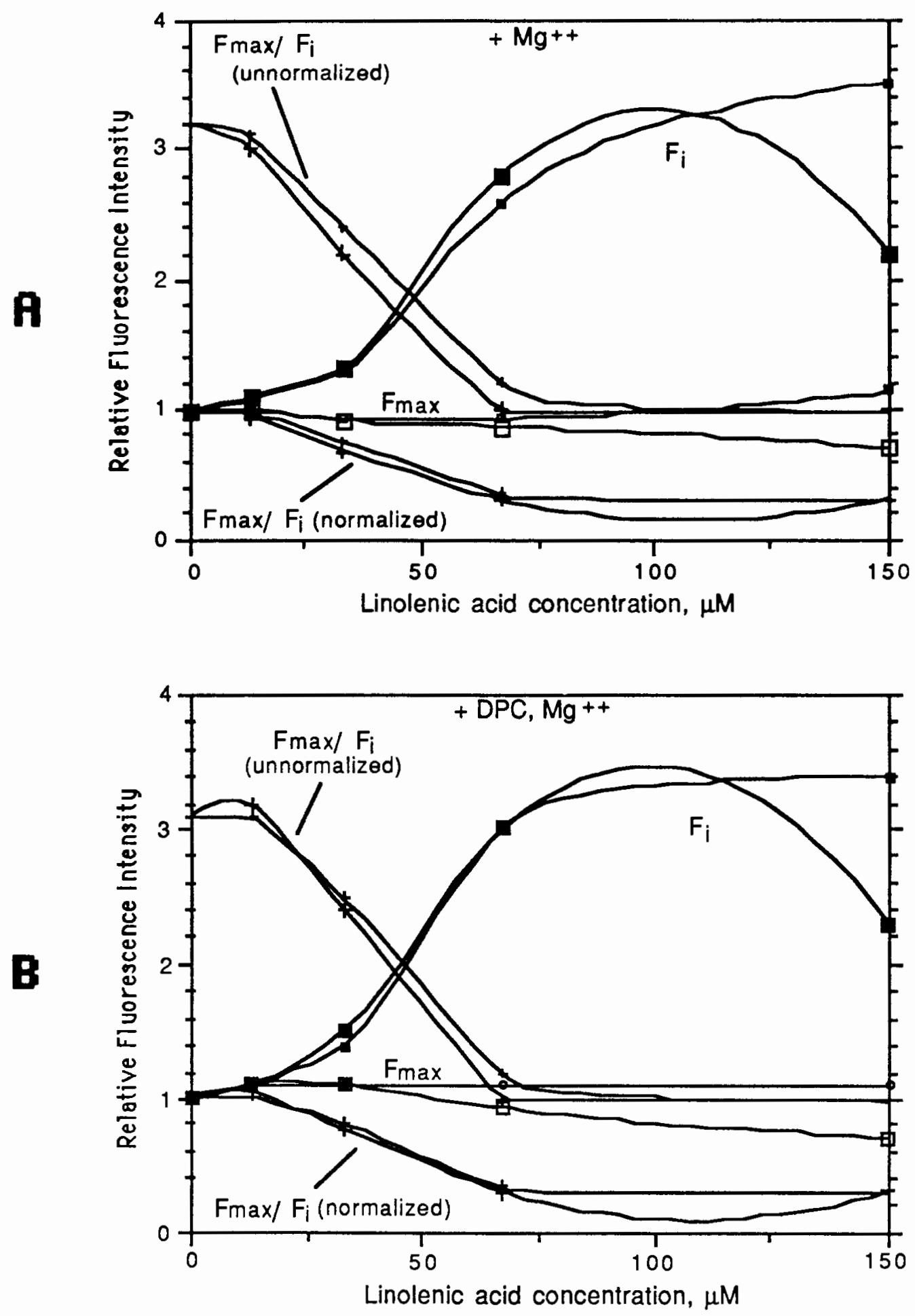

Figure 13. Fluorescence intensity vs. linolenic acid concentration in the presence of $10 \mathrm{mM} \mathrm{Mg}^{2+},+/$ - DPC. Smaller symbols represent a $20 \mathrm{sec} \mathrm{LA}$ incubation period, larger symbols a $5 \mathrm{~min}$ incubation. $\mathrm{pH}=7.6$ (HEPES).

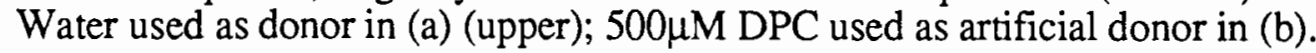




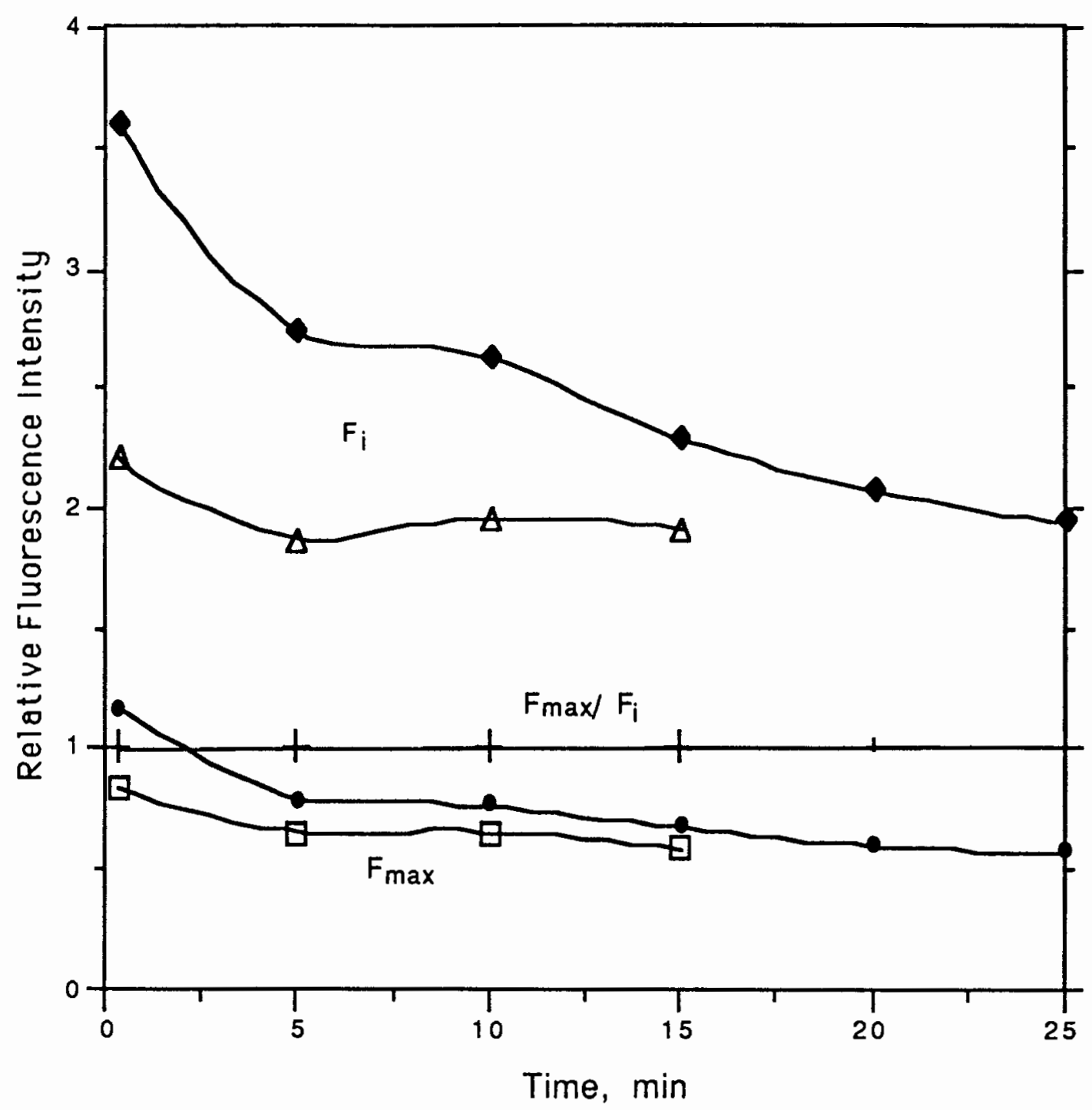

Figure 14. Relative fluorescence intensity vs. time of incubation with $150 \mu \mathrm{M}$ linolenic acid, $+/-\mathrm{Mg}^{2+} . \mathrm{pH}=7.6$ (HEPES). Open symbols = no $\mathrm{Mg}^{2+}$; closed symbols $=$ plus $20 \mathrm{mM} \mathrm{Mg}^{2+}$. 

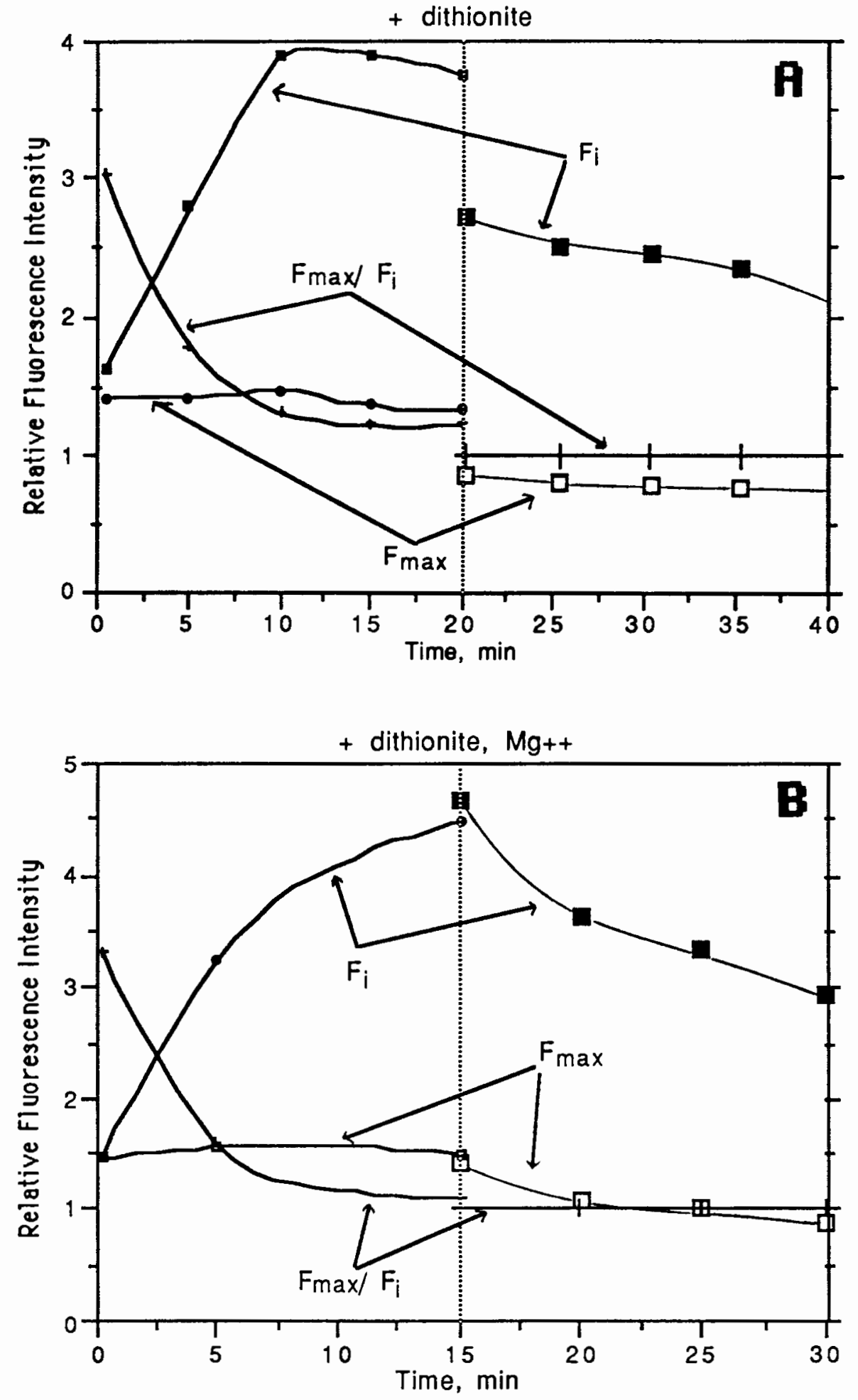

Figure 15. Relative fluorescence intensity vs. incubation time with dithionite, $+/$ - linolenic acid and $\mathrm{Mg}^{2+} . \mathrm{pH}=7.6$ (HEPES). Smaller symbols for dithionite; larger symbols for dithionite plus $150 \mu \mathrm{M}$ LA. Vertical line indicates point of LA addition to dithionite-treated sample. No $\mathrm{Mg}^{2+}$ present for (a) (upper); $20 \mathrm{mM} \mathrm{Mg}^{2+}$ present for (b). 


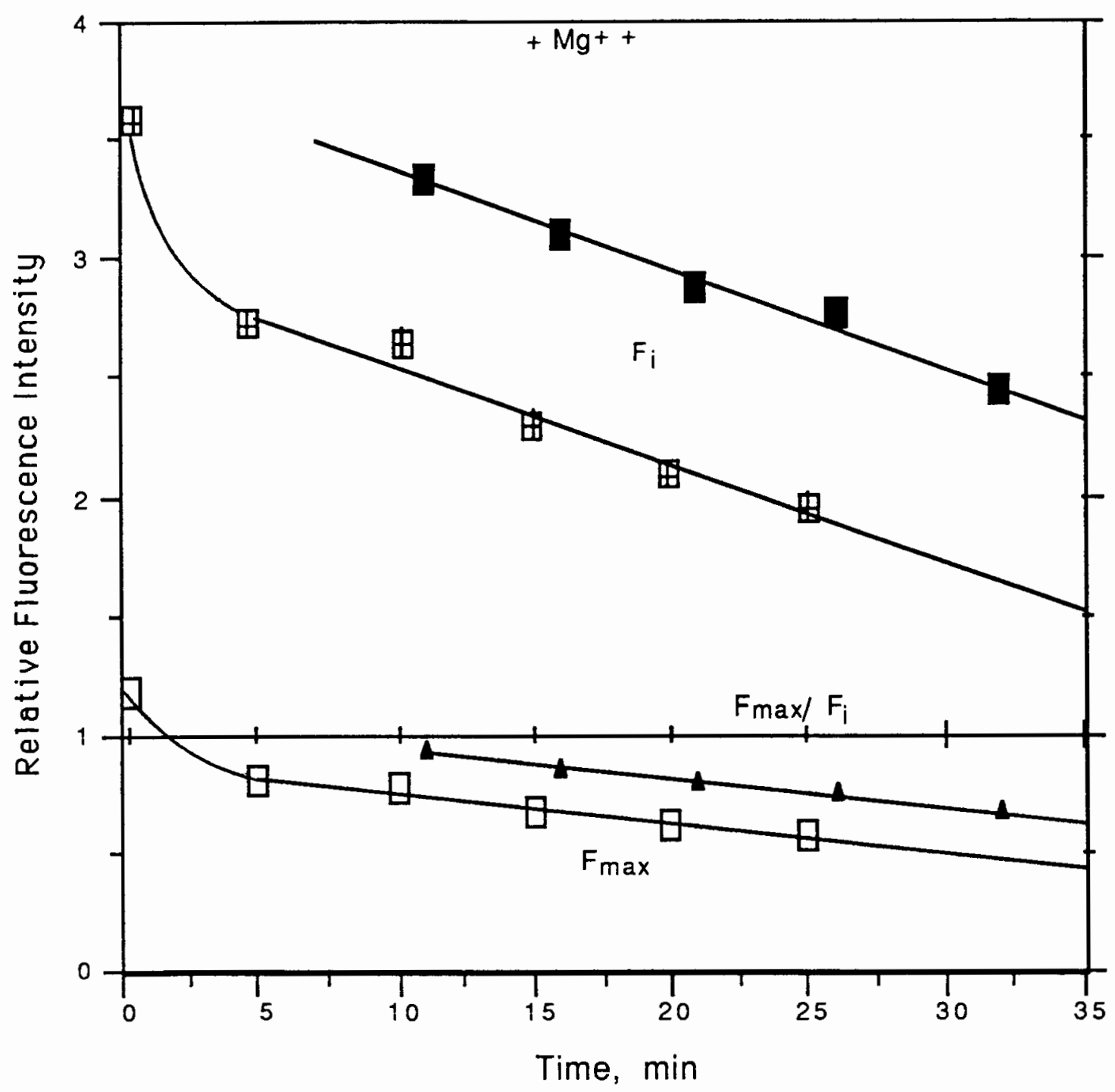

Figure 16. Relative fluorescence intensity vs. incubation time with $150 \mu \mathrm{M}$ linolenic acid in the presence of $20 \mathrm{mMMg}^{2+},+/$ - dithionite. $\mathrm{pH}=7.6$ (HEPES). Closed symbols = LA; open symbols $=$ LA plus dithionite $\left(\mathrm{F}_{\max } / \mathrm{F}_{\mathrm{i}}=1\right.$ in both cases). 

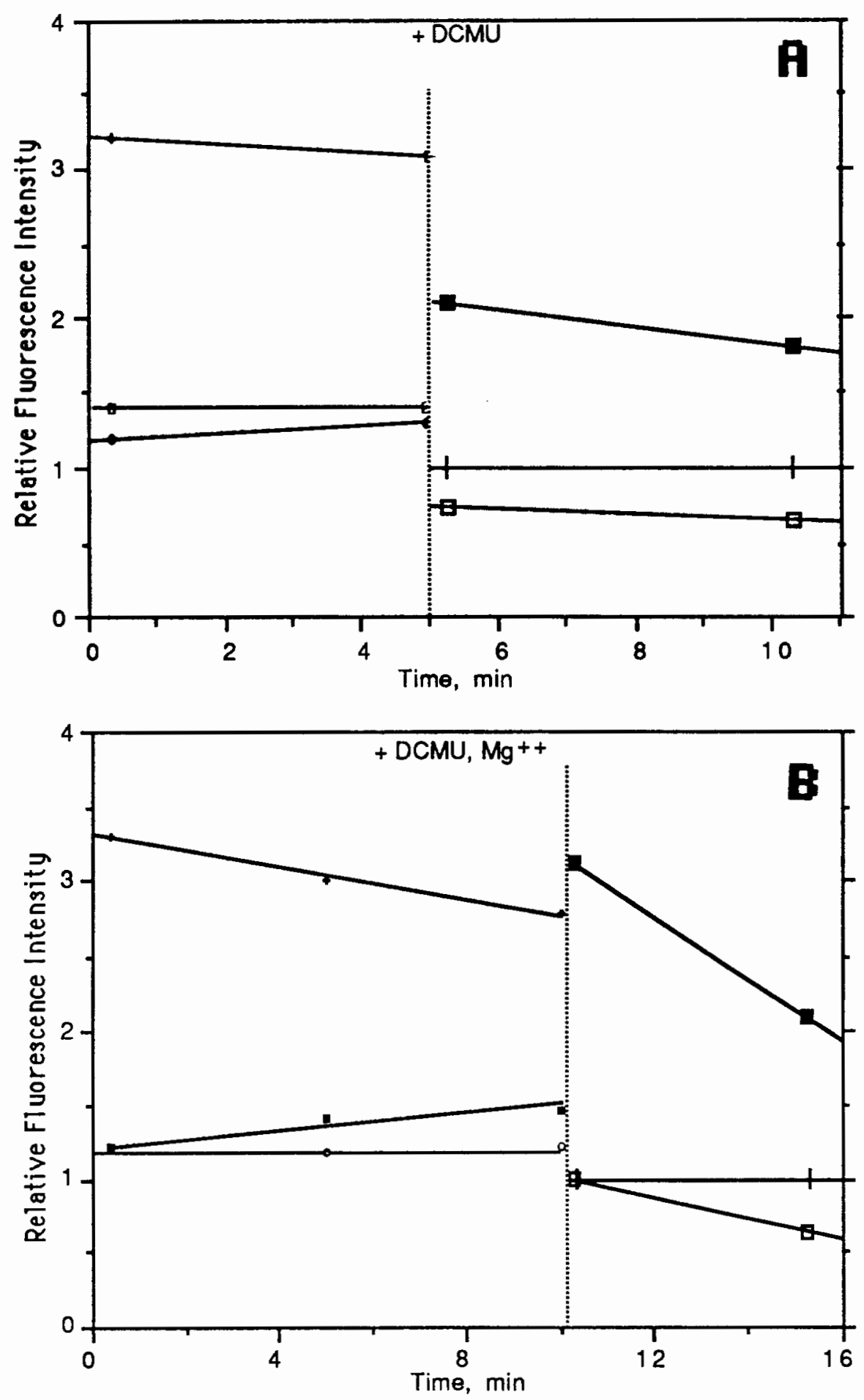

Figure 17. Relative fluorescence intensity vs. incubation time with $5 \mu \mathrm{M}$

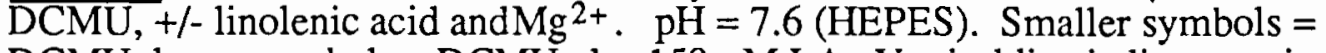
DCMU; larger symbols $=$ DCMU plus $150 \mu \mathrm{M}$ LA. Vertical line indicates point of LA addition to DCMU-treated sample. $\mathrm{No} \mathrm{Mg}^{2+}$ present for (a) (upper); 20 $\mathrm{mM} \mathrm{Mg}^{2+}$ present for (b). Closed square $=\mathrm{F}_{\mathrm{i}}$; open square $=\mathrm{F}_{\max } ;$ cross $=$ $\mathrm{F}_{\max } / \mathrm{F}_{\mathrm{i}}$. 


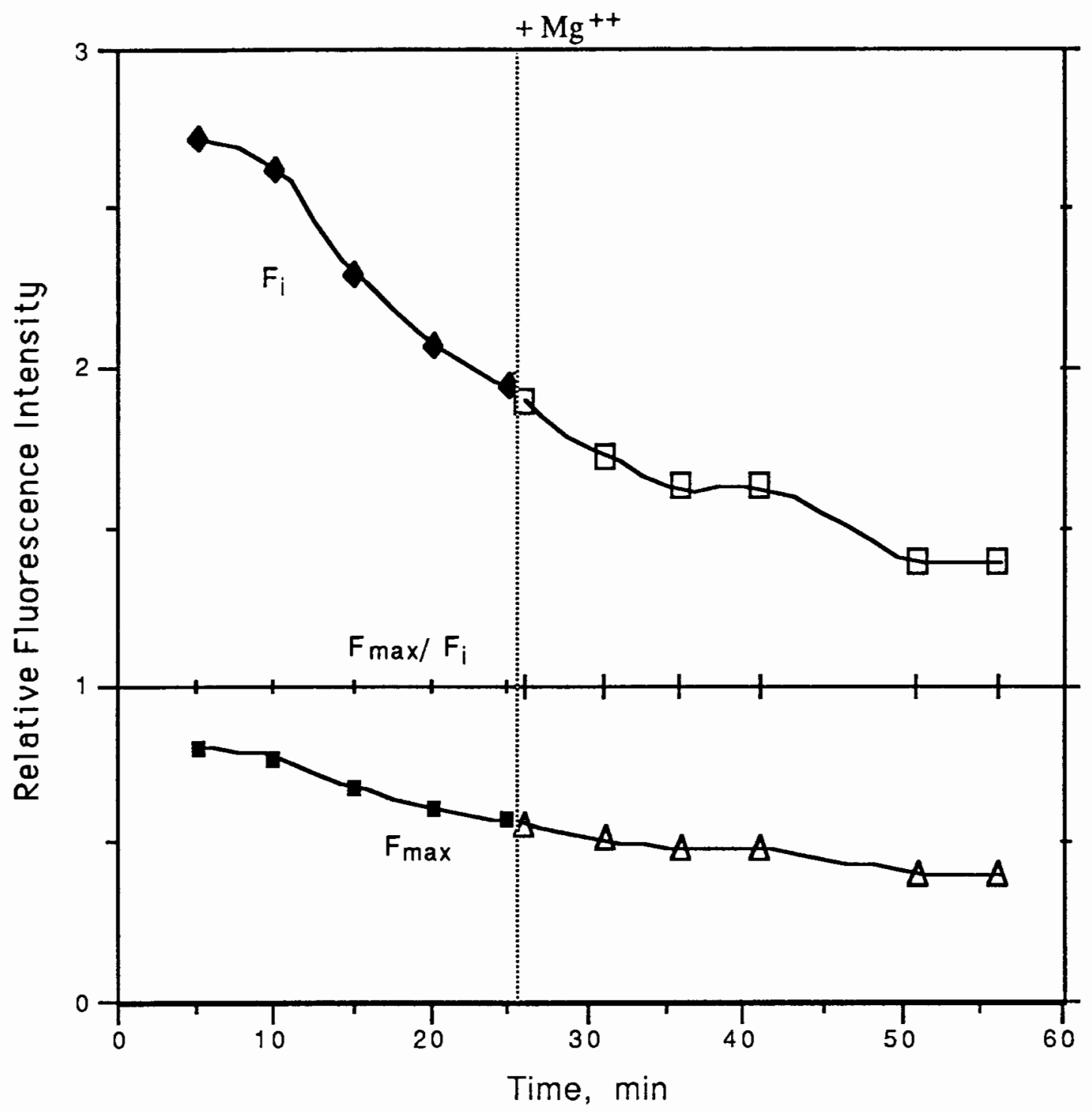

Figure 18. Relative fluorescence intensity vs. incubation time with $150 \mu \mathrm{M}$ linolenic acid and $20 \mathrm{mM} \mathrm{Mg}+,+/-$ DCMU. $\mathrm{pH}=7.6$ (HEPES). Closed symbols $=\mathrm{LA}$; open symbols $=\mathrm{LA}$ plus $5 \mu \mathrm{M} \mathrm{DCMU}$. Vertical line indicates point where DCMU added to LA-treated sample. 

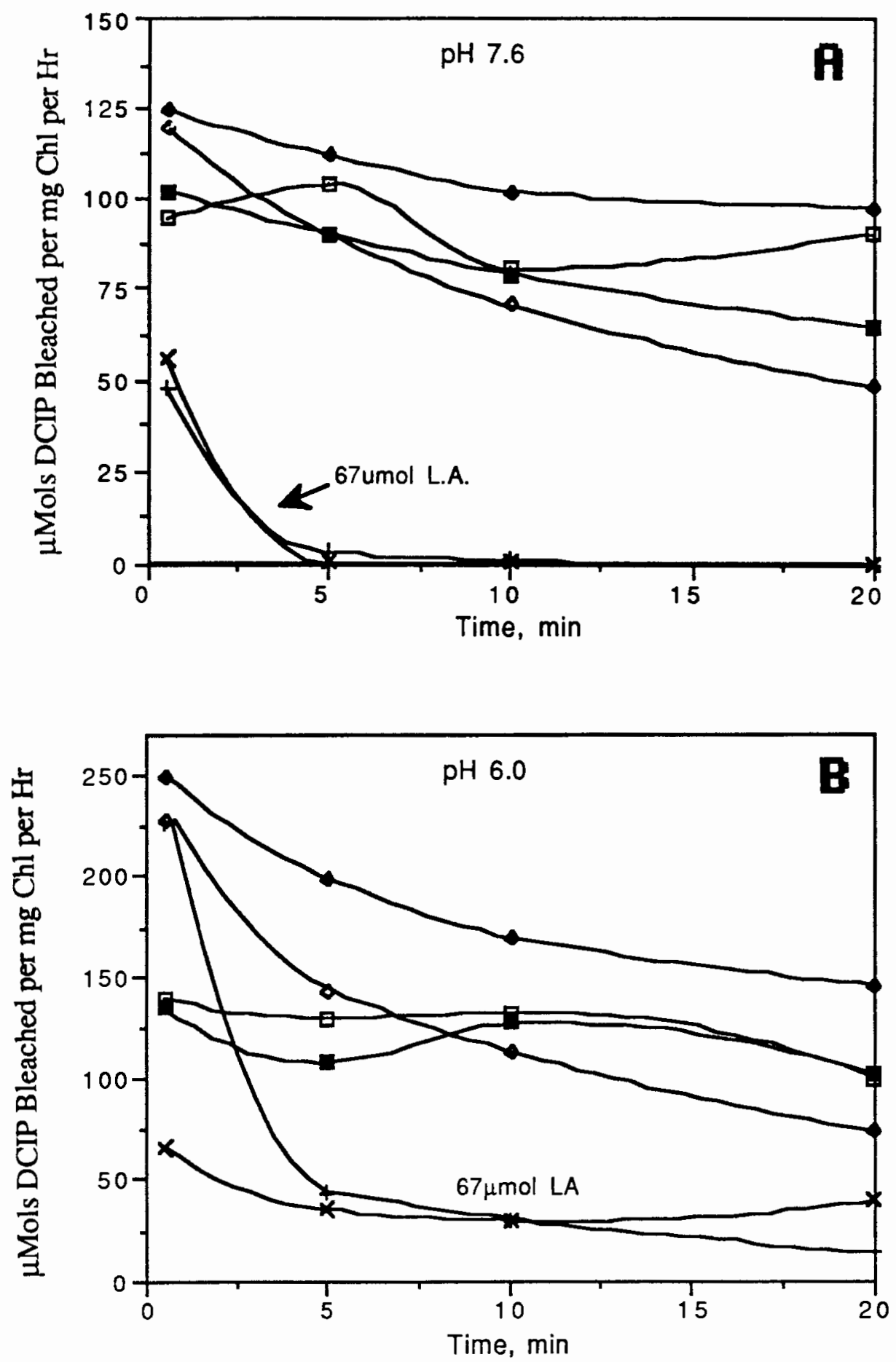

Figure 19. Average DCIP reduction rates vs. time for differing concentrations of linolenic acid, +/- DPC. $\mathrm{pH}=7.6$ (HEPES) for (a) (upper) and 6.0 (HEPES) for (b). Excitation wavelength $=650 \mathrm{~nm} . \quad \square=$ control; $\bullet=$ control + DPC; $\quad=33 \mu \mathrm{M} \mathrm{LA} ; \quad \diamond=33 \mu \mathrm{M} \mathrm{LA}+\mathrm{DPC} ; \quad \mathrm{X}=67 \mu \mathrm{M} \mathrm{LA} ;$ $t=67 \mu \mathrm{MLA}+\mathrm{DPC}$. 

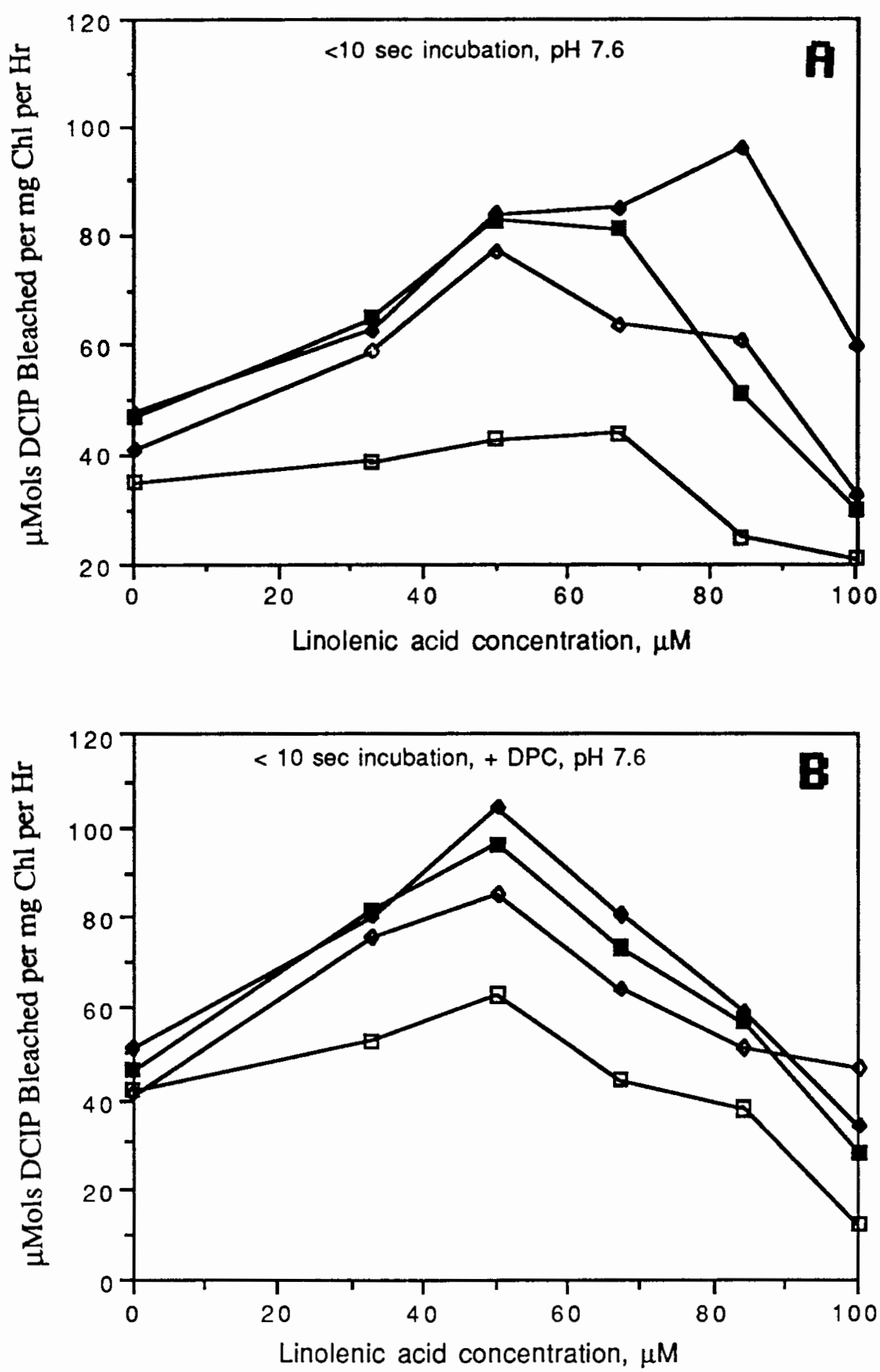

Figure 20. Average DCIP reduction rates vs linolenic acid concentration (10 sec incubation) at differing excitation light intensities, $+/$ - DPC. Excitation wavelength $=650 \mathrm{~nm}$. $\mathrm{pH}=7.6$ (HEPES). Water used as donor in (a) (upper); $500 \mu \mathrm{M} \mathrm{DPC}$ used as artificial donor in (b). $\quad=100 \% \mathrm{~T} ; \boldsymbol{\square}=$ $55 \% \mathrm{~T} ; \quad \diamond=34 \% \mathrm{~T} ; \quad \square=12 \% \mathrm{~T}$. 


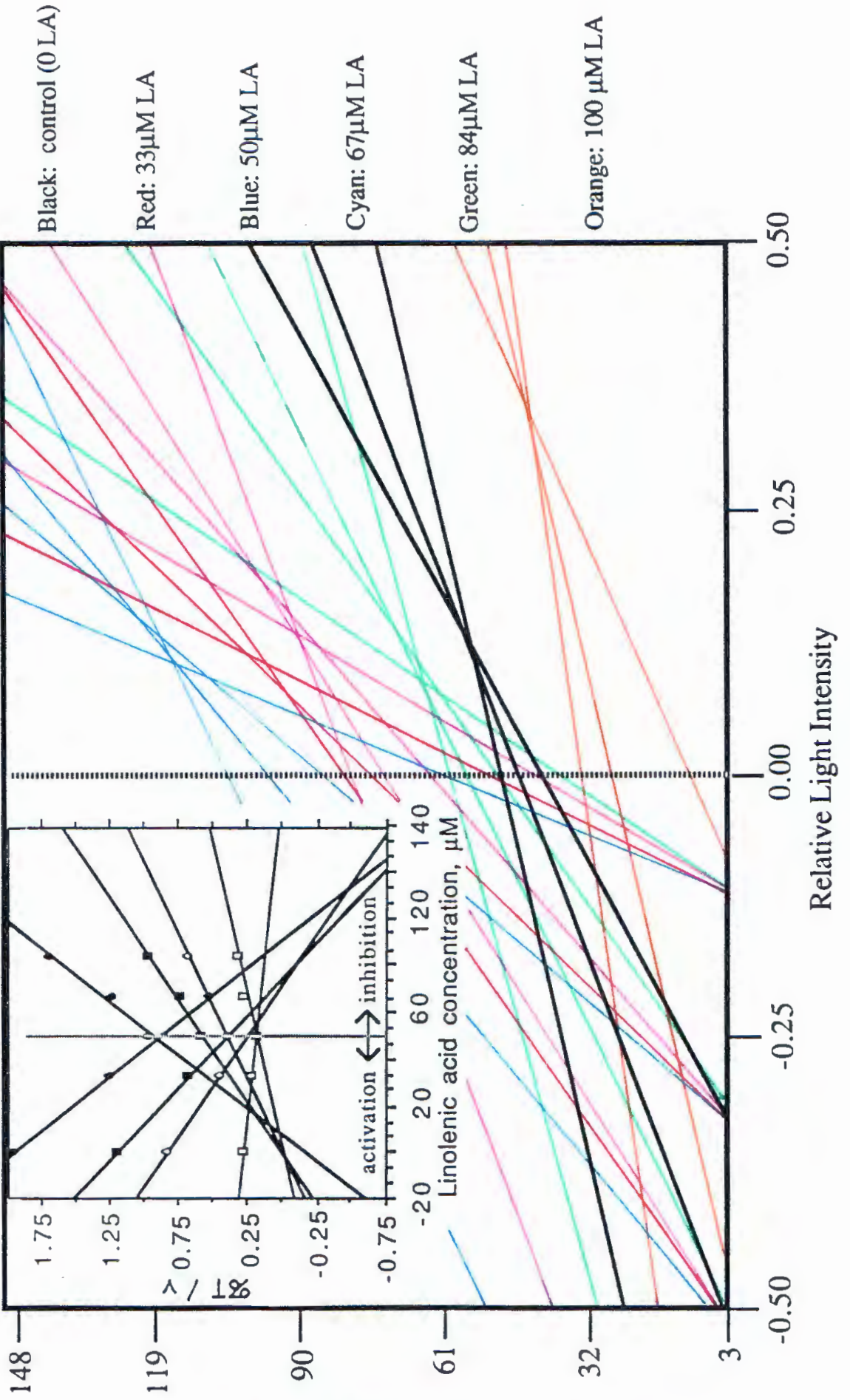

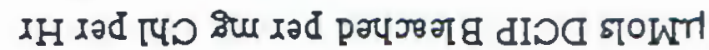

范采

垔

ㄷํㅇํํ

氙望. 矛要

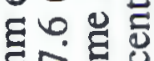
"II

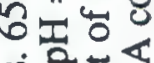
的吉手 不品 ن 갑 동하웅 可产高 곤 11.0 . 㶽 过是 呵客 돵ㅎㅇ 츠으.․․․․ 응

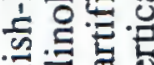

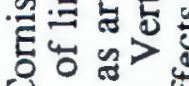
O) of. 응 할츨 등 혀에

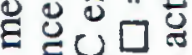

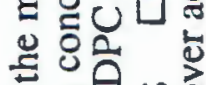

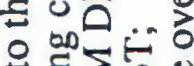

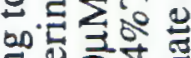
og

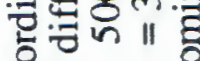
迎。

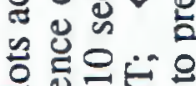

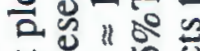

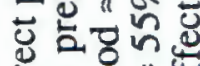

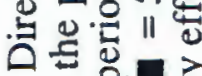
तi ov 年

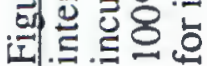



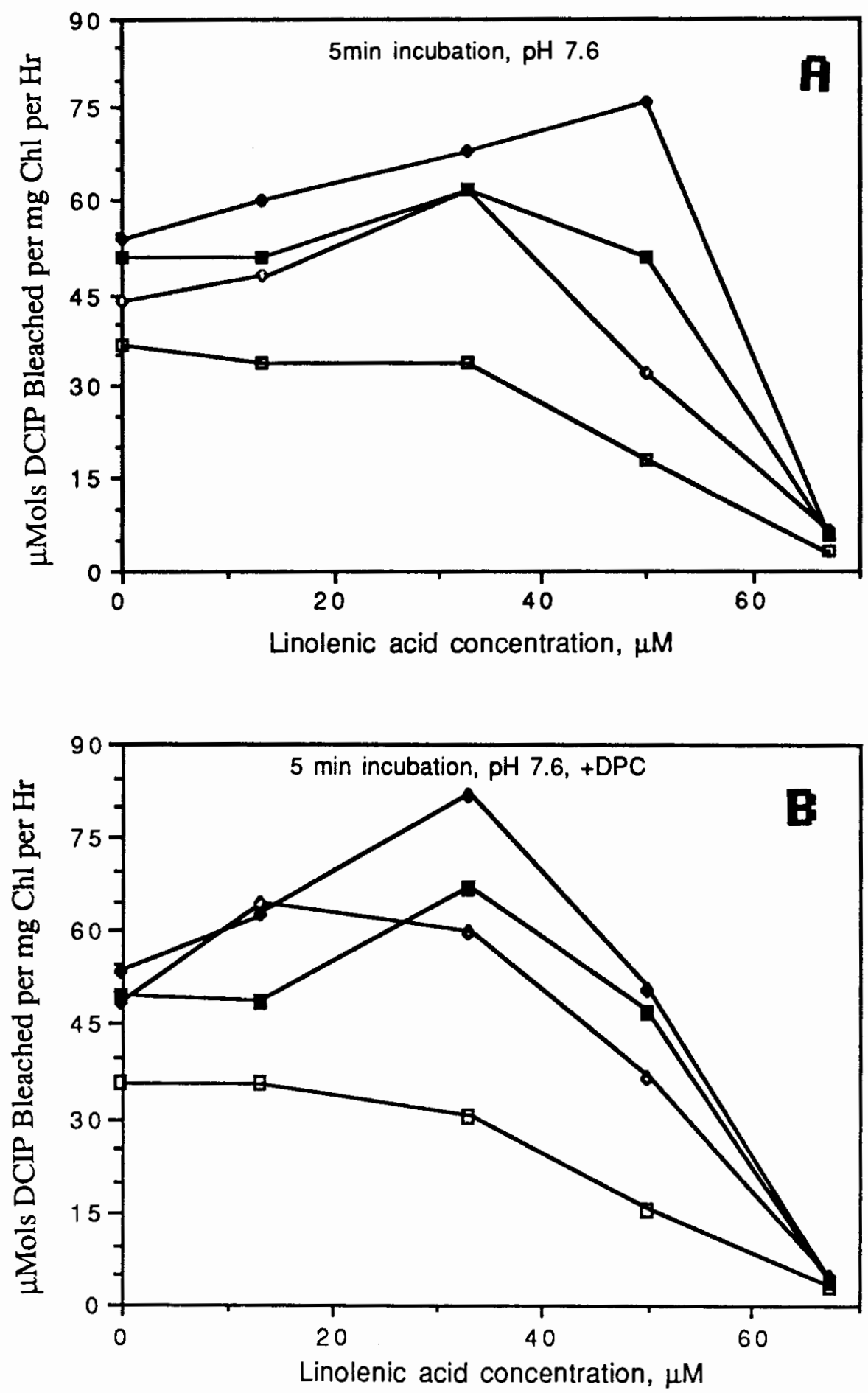

Figure 22. Average DCIP reduction rates vs. linolenic acid concentration (5 min incubation) at differing excitation light intensities, +/- DPC. Excitation wavelength $=650 \mathrm{~nm} . \mathrm{pH}=7.6$ (HEPES). Water used as donor in (a) (upper); $500 \mu \mathrm{M}$ DPC as an artificial donor in (b). $\quad=100 \% \mathrm{~T} ; \quad \boldsymbol{\square}=55 \% \mathrm{~T}$; $\Delta=34 \% \mathrm{~T} ;$ 口 $=12 \% \mathrm{~T}$. 

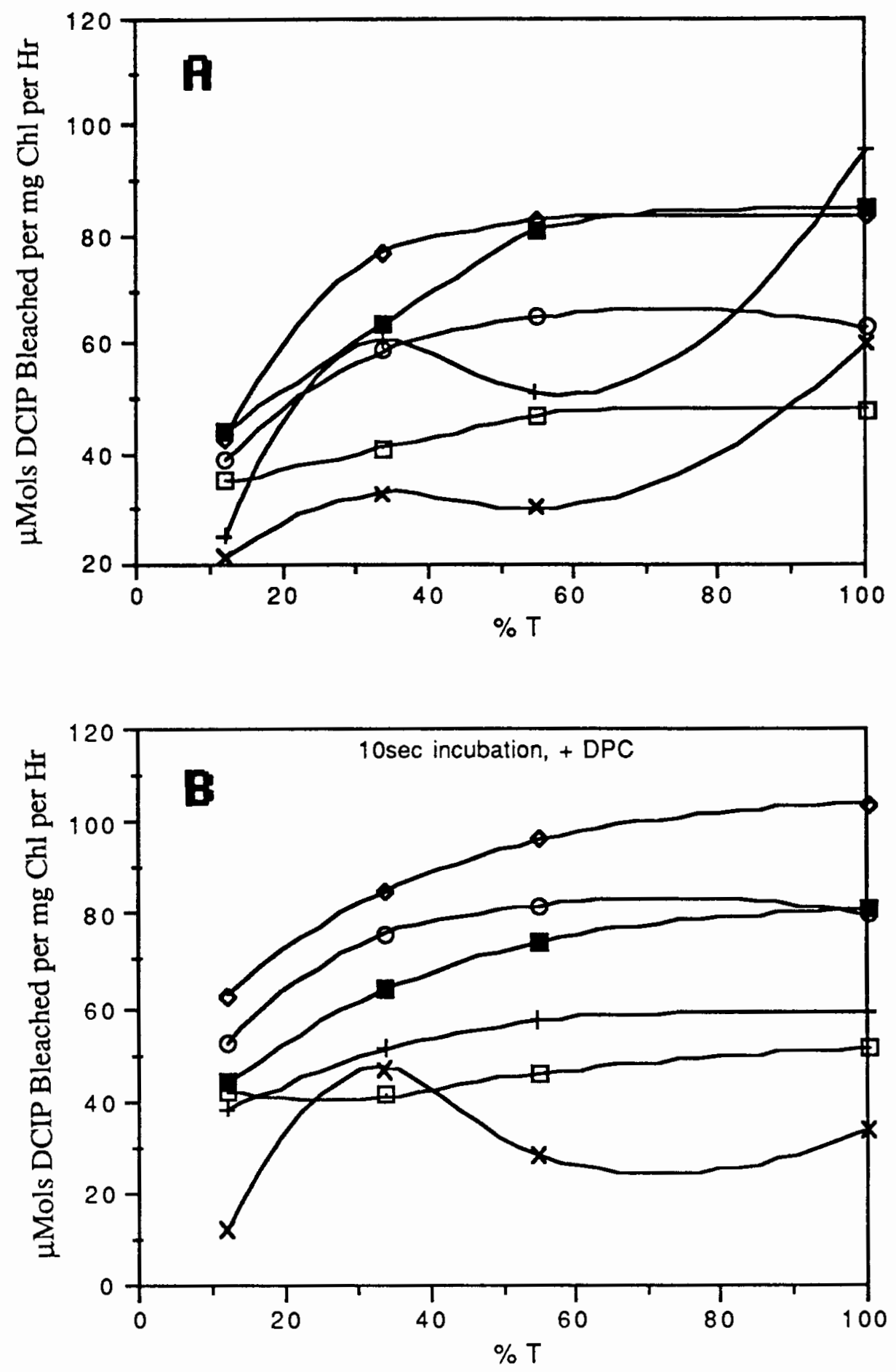

Figure 23. DCIP reduction rates vs. $650 \mathrm{~nm}$ excitation light intensity at differing concentrations of linolenic acid (10 sec incubation), +/- DPC. $\mathrm{pH}=$ 7.6 (HEPES). Water used as donor in (a)(upper); $500 \mu \mathrm{M}$ DPC used as an artificial donor in (b). $\quad \square=$ control $(0 \mathrm{LA}) ; \quad 0=33 \mu \mathrm{M} \mathrm{LA} ; \quad \diamond=50 \mu \mathrm{M}$ $\mathrm{LA} ; \quad \boldsymbol{\square}=67 \mu \mathrm{MLA} ; \quad+=84 \mu \mathrm{MLA} ; \quad \boldsymbol{x}=100 \mu \mathrm{MLA}$. 

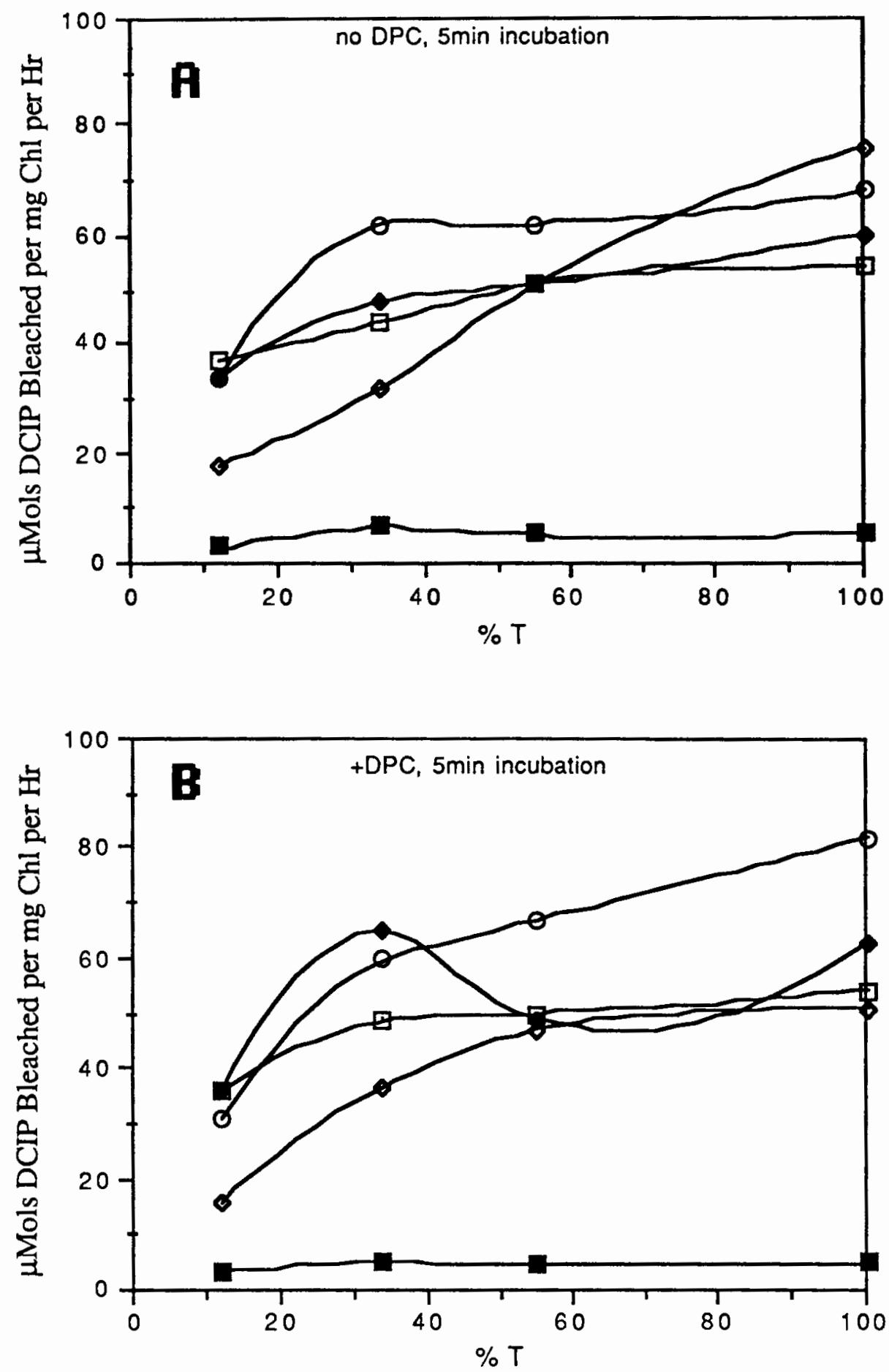

Figure 24. DCIP reduction rates vs. $650 \mathrm{~nm}$ excitation light intensity at differing concentrations of linolenic acid ( 5 min incubation), $+/$ - DPC. $\mathrm{pH}=$ 7.6 (HEPES). Water used as donor in (a)(upper); $500 \mu \mathrm{M}$ DPC used as an artificial donor in (b). $\quad \square=$ control $(0 \mathrm{LA}) ; \quad \forall=13 \mu \mathrm{M} \mathrm{LA} ; \quad 0=33 \mu \mathrm{M}$ $\mathrm{LA} ; \diamond=50 \mu \mathrm{M} \mathrm{LA} ; \quad \square=67 \mu \mathrm{M} \mathrm{LA}$. 


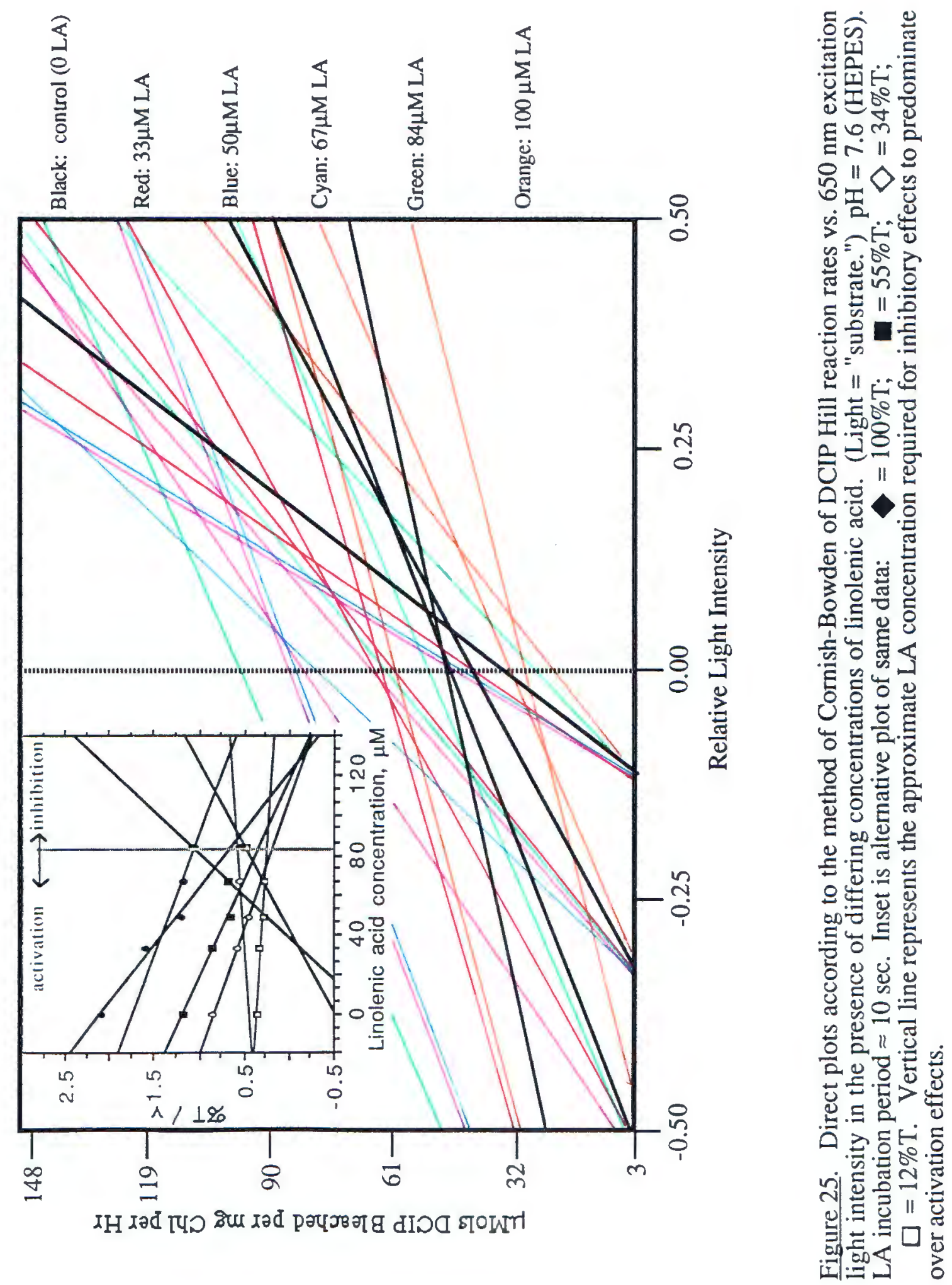




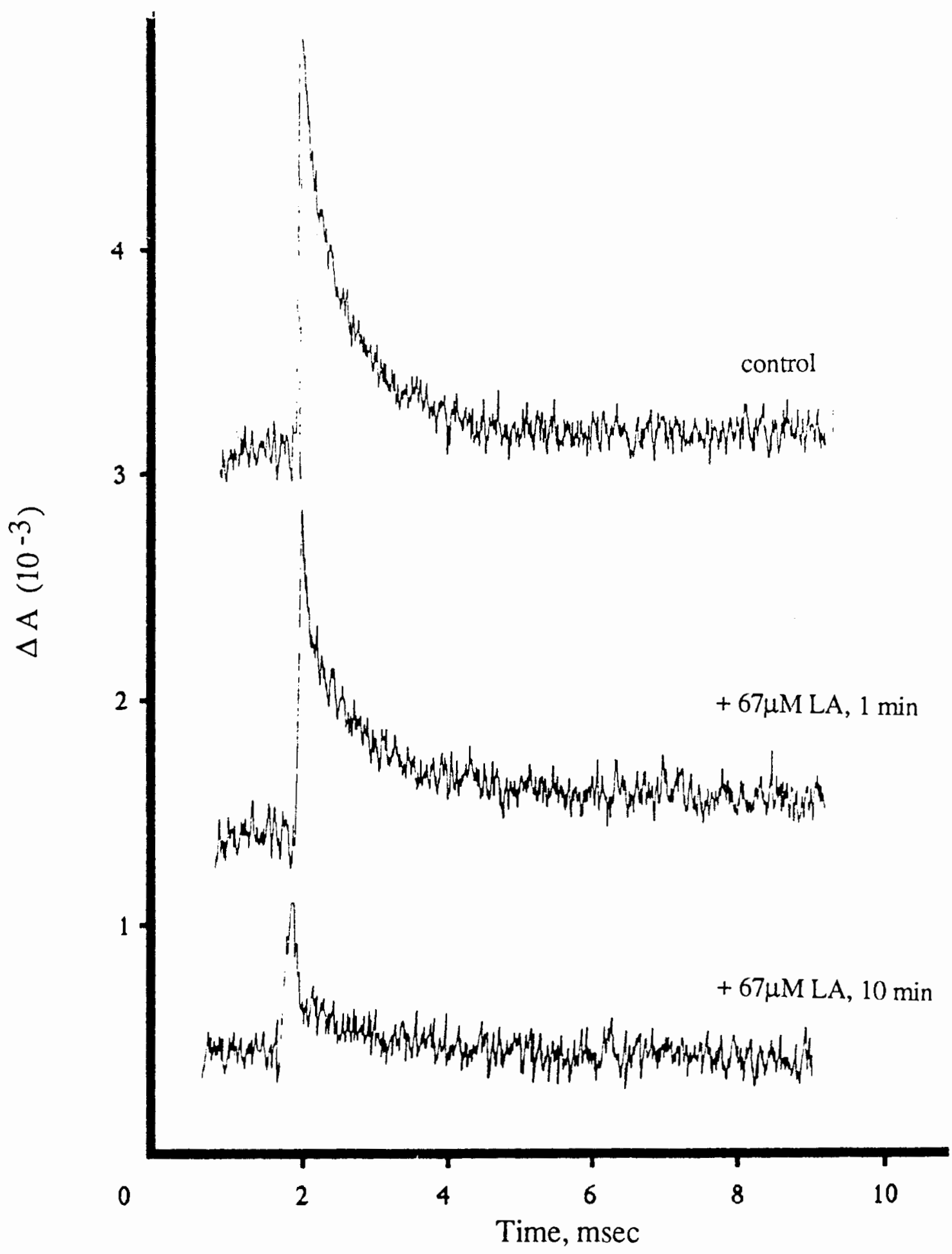

Figure 26. Re-reduction kinetics of photooxidized P680 at pH 7.6 (HEPES). Measured at $820 \mathrm{~nm}$ using everted wheat thylakoids containing PSII. Excitation accomplished using saturating $650 \mathrm{~nm}$ pulsed laser. Top curve is for the control (no additions). Lower two curves recorded after incubation with $67 \mu \mathrm{M}$ linolenic acid for 1 and $10 \mathrm{~min}$, respectively. 

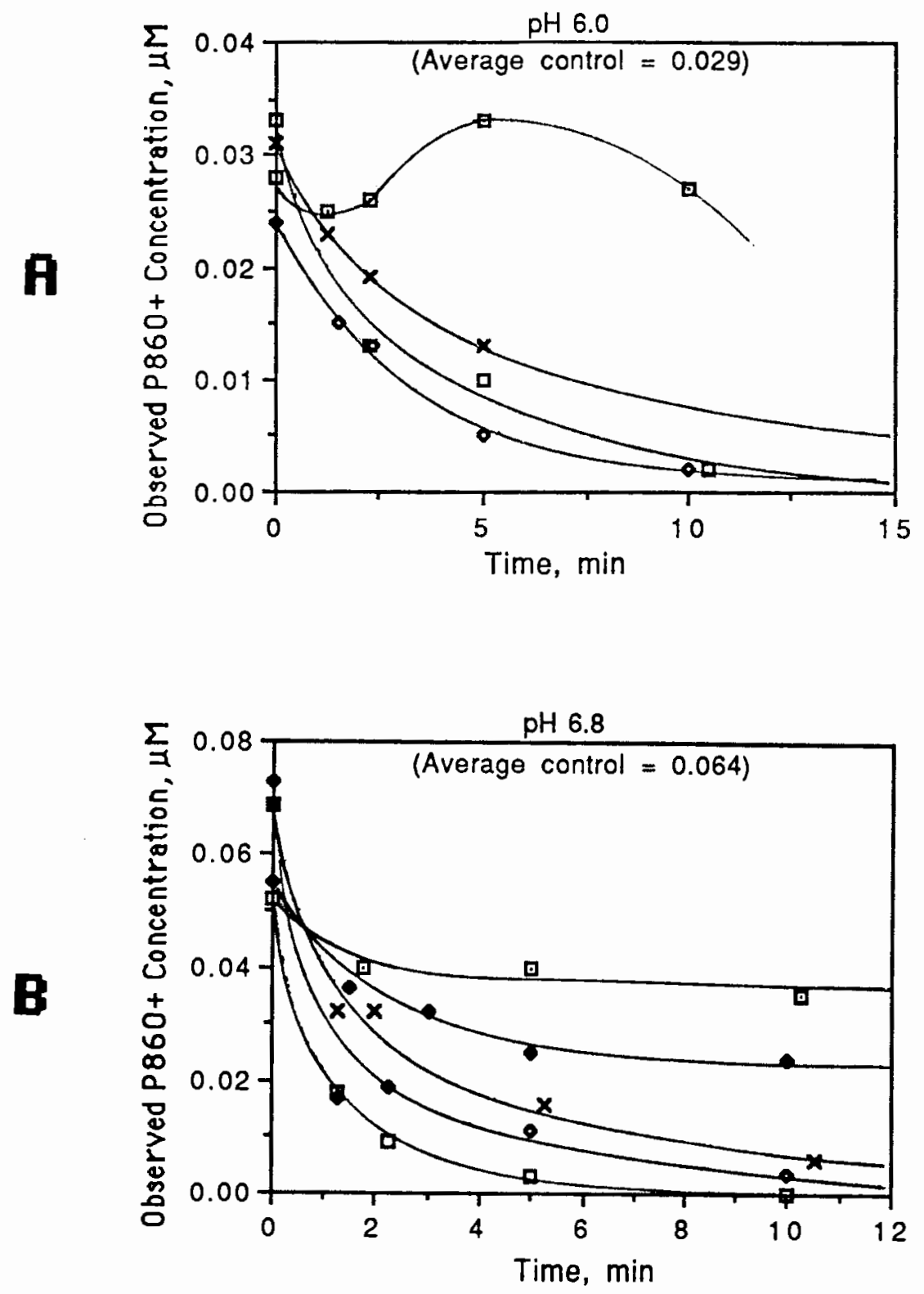

Figure 27. Observed $\mathrm{P} 680^{+}$concentration vs. time at various $\mathrm{pH}$ in the presence of differing concentrations of linolenic acid. $\mathrm{pH}=6.0$ and 6.8 (HEPES), respectively, for (a) (upper) and (b) (lower). $\mathrm{pH}=7.6$ (HEPES) for (c) (following page). Sample = everted wheat thylakoids containing PSII. Concentrations determined from peak height of $820 \mathrm{~nm}$ absorption transients. Average control values were measured prior to $L A$ addition and covered a period of $1-5 \mathrm{~min} . \quad \square=13 \mu \mathrm{M} \mathrm{LA}$; $100 \mu \mathrm{MLA} ; \diamond=150 \mu \mathrm{M} \mathrm{LA}$. 


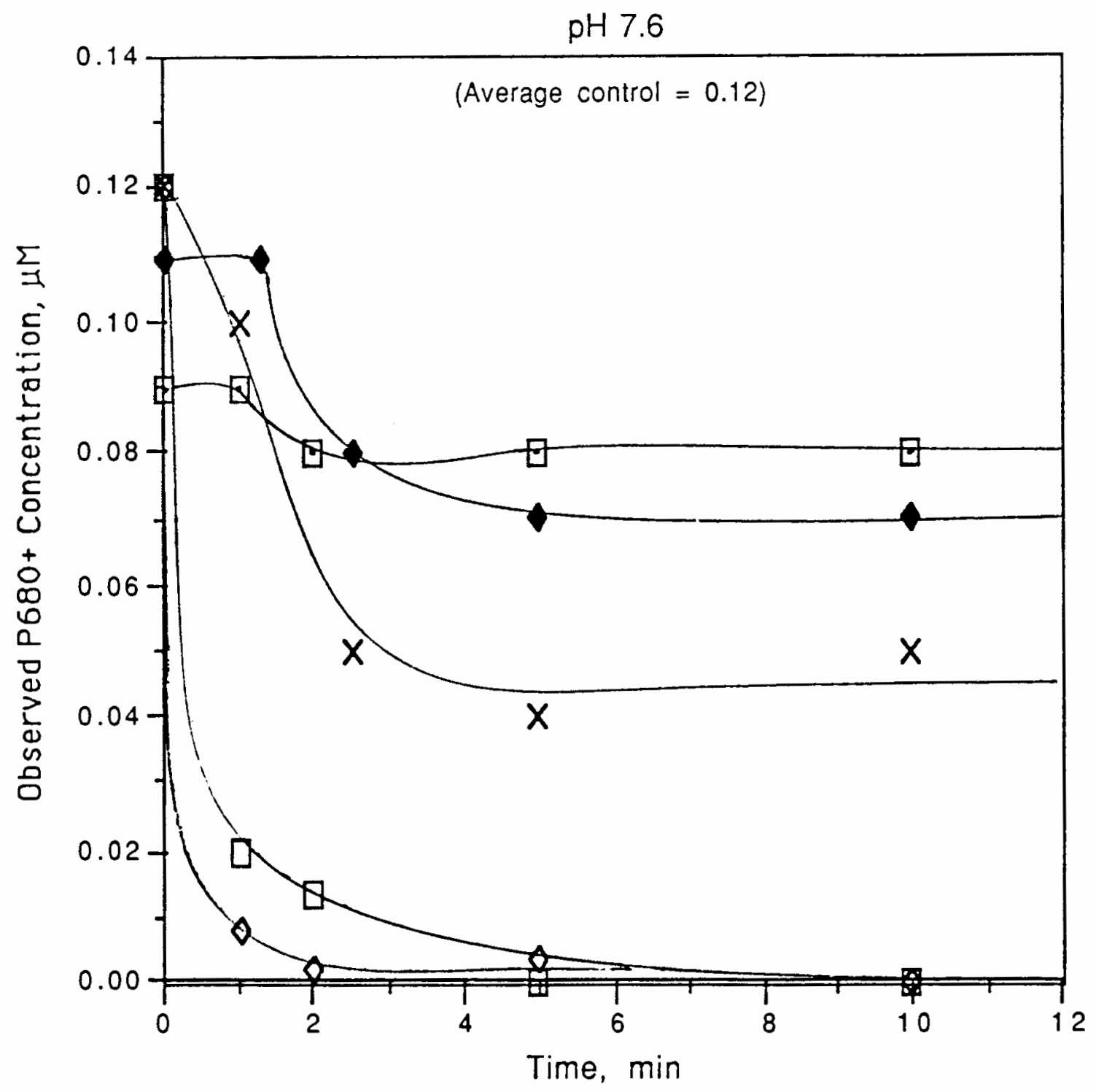

Figure 27(c). Observed $\mathrm{P} 680+$ concentration vs. time at $\mathrm{pH} 7.6$ in the presence of differing concentrations of linolenic acid.

$33 \mu \mathrm{MLA} ; \quad x=67 \mu \mathrm{MLA} ; \quad \square=100 \mu \mathrm{MLA} ; \diamond=150 \mu .1 / \mathrm{LA}$. 


\section{APPENDIX B}

FLUORESCENCE DECAY DATA IN THE PRESENCE OF LINOLENIC ACID 
Table III on the following page illustrates the calculated lifetimes and relative yields of fluorescence decay components measured at $\mathrm{F}_{o}$ and $\mathrm{F}_{\max }$ in spinach chloroplasts according to Tabbutt, Golbeck and Sauer, 1985 (personal communication from J.H.Golbeck). Results show the presence of three decay components in control and DCMU-treated samples which slow significantly from $F_{o}$ to $F_{\max }$ as the lifetime and relative yield of the slow component increases. LA-treated samples, on the other hand, exhibit behavior which is essentially independent of light intensity, thus eliminating the distinction between $\mathrm{F}_{o}$ and $\mathrm{F}_{\max }$. The group concludes that $i$ ) LA does not inhibit charge separation / recombination in PSII; (ii) LA in the dark produces a state similar to DCMU at $\mathrm{F}_{\max }$ by blocking electron transport between $\mathrm{Ph}$ and $\mathrm{Q}_{\mathrm{A}}$; (iii) $\mathrm{LA}$ inhibition can be reversed with bovine serum albumin (BSA). 


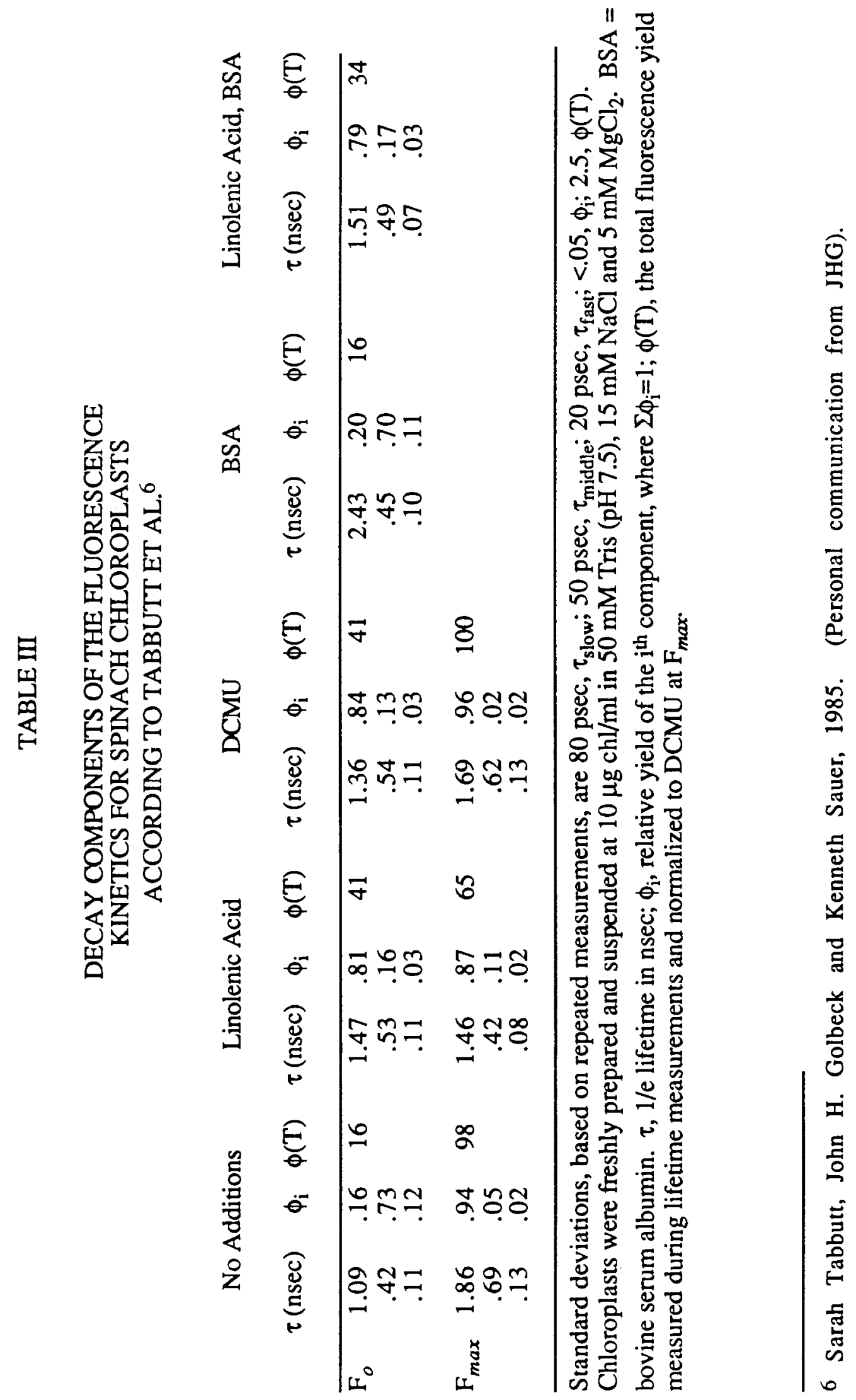

